\title{
Indicador de alerta temprana para la detección de vulnerabilidades de los fondos de inversión colectiva (FIC)
}

\section{Early warning indicator for detection of vulnerabilities of collective investment funds (FIC)}

\section{Ana María Rendón-González}

* Profesional en Finanzas y Comercio Internacional, Universidad del Rosario; magíster en Finanzas, Universidad Externado de Colombia. Super intendencia Financiera de Colombia. Profesional de riesgos de mercado y liquidez. Bogotá (Colombia). [anamariarend@gmail.com], [ORCID: 0000-0002-3986-977X].

Artículo recibido el 20 de noviembre de 2020.

Aceptado el 15 de enero de 2021.

Para citar este artículo:

Rendón-González, A. M. (2020). Indicador de alerta temprana para la detección de vulnerabilidades de los fondos de inversión colectiva (FIC). ODEON, 19, 31-79.

DOI: https://doi.org/10.18601/17941113.n19.03 


\section{Resumen}

Dada la alta popularidad que han venido ganando los fondos de inversión colectiva (FIC) y su creciente participación en el mercado financiero, tanto en monto de inversiones como en número de inversionistas, es necesario contar con herramientas que permitan una adecuada y oportuna identificación de los riesgos, con el fin de prevenir una afectación a la estabilidad del sistema financiero. Sin embargo, la literatura en cuanto a alertas tempranas de estos fondos es escasa, ya que la mayoría de trabajos están enfocados en el sector bancario.

El objetivo de este documento es desarrollar un indicador de alerta temprana que permita la identificación de vulnerabilidades de los FIC, con una ventana de tiempo suficiente a fin de que los hacedores de política implementen medidas para su contención. Para la consecución del objetivo planteado, se adaptará al escenario de los FIC en Colombia una medida de alerta temprana ampliamente conocida en el sector bancario, como es el caso del Indicador de Riesgo Sistémico Doméstico (d-SRI) del Banco Central Europeo.

Los resultados obtenidos muestran que ante un choque de una desviación estándar en el indicador de alertas tempranas de los FIC (IATE FIC), se espera una caída de 1,4 puntos porcentuales en el crecimiento trimestral del valor neto de los FIC (VNF) en los seis meses posteriores a la ocurrencia del choque. Los hallazgos encontrados en el presente trabajo representan un avance importante en la detección de vulnerabilidades de los fondos de inversión y pueden ser de gran ayuda para los hacedores de política en la toma de decisiones ante escenarios de incertidumbre en el comportamiento futuro de la industria de FIC.

Palabras clave: fondos de inversión colectiva; indicadores de alerta temprana. Clasificación JEL: G00, G11, G18.

\section{Abstract}

Given the high popularity that collective investment funds (CIFs) have been gaining and their growing participation in the financial market, both in terms of the amount of investments and the number of investors, it is necessary to have tools that allow for adequate and timely identification of risks, in order to prevent an effect on the stability of the financial system. However, there is a lack of literature on early warnings for these funds, since most of the papers are focused on the banking sector.

This document aims to develop an early warning indicator that allows the identification of CIF vulnerabilities, with enough time of anticipation for policymakers to implement measures for their containment. In order to achieve this 
objective, a widely known early warning measure in the banking sector, such as the European Central Bank's Domestic Systemic Risk Indicator (d-SRI), will be adapted to the CIF scenario in Colombia.

The results obtained show that in the event of a shock of one standard deviation in the CIF early warning indicator (IATE FIC), a drop of 1.4 percentage points in the quarterly growth of the fund's net value (FNV) is expected in the six months following the occurrence of the shock. The findings showed in this work represent an important advance in the detection of vulnerabilities of investment funds and can be of great help to policymakers in making decisions under scenarios of uncertainty in the future behavior of the CIF industry.

Key words: Collective investment funds; early warning indicators.

JEL classification: G00, G11, G18.

\section{Introducción}

En los últimos años, los vehículos de inversión colectiva han ganado participación e importancia, y se han consolidado como uno de los principales actores en los mercados financieros globales. Sin embargo, al analizar la literatura en cuanto a los efectos de esta industria sobre la estabilidad financiera, los trabajos son escasos ya que el enfoque principal se centra en los efectos de los establecimientos de crédito, principalmente el sector bancario.

Con la evolución de los mercados financieros, los fondos de inversión se han convertido en un agente importante del mercado dados los altos niveles de activos administrados que han alcanzado ${ }^{1}$ y la diversificación de activos en los que están involucrados, obteniendo el nivel de agentes sistémicos en algunos mercados o países. Por ejemplo, los activos administrados de los fondos en Brasil fueron equivalentes aproximadamente a $123 \%$ del PIB al cierre de 2019 (FIAFIN, n.d.), mientras que para el caso de Estados Unidos y Canadá esta participación ascendió al 70 y $54 \%$, respectivamente, con cifras a 2014 (Ramírez et al., 2015).

Colombia no ha sido ajena a esta realidad, entre 2008 y 2019, los fondos de inversión colectiva (FIC) han pasado de administrar US $\$ 12$ mil millones a US $\$ 35$ mil millones. De igual manera, el número de adherentes se ha incrementado

1 En 2017 alcanzaron US\$3.578 billones de acuerdo con U.S. Securities and Exchange Commission-SEC (Division of Investment Management Risk and Examinations Office Risk and Examinations Office, 2017), duplicándose desde 2010 y triplicándose desde 2004. 
significativamente al pasar de 569 mil a 1,9 millones, de los cuales en su gran mayoría corresponden a personas naturales, que encuentran en dichos fondos una fuente de diversificación de sus recursos y un punto de entrada para invertir en el mercado de capitales.

Dada la alta popularidad que han venido ganando los FIC y su creciente participación en el mercado financiero, es necesario contar con herramientas que permitan una adecuada y oportuna identificación de los riesgos a los cuales se encuentran expuestos dichos fondos. A su vez, teniendo en cuenta que cada vez son más las personas que depositan su confianza y sus recursos en los FIC, una afectación a los mismos puede acarrear serios problemas al funcionamiento y la estabilidad del sistema financiero.

Por las razones enunciadas, es de vital importancia tanto para la estabilidad del sistema financiero colombiano como para la confianza de los consumidores, herramientas que permitan evidenciar y anticipar las vulnerabilidades de los FIC.

Ante las necesidades expuestas previamente, el objetivo de este documento es presentar un indicador de alerta temprana para la detección de fragilidad financiera de los FIC en Colombia, a partir de la adaptación del indicador de riesgo sistémico (d-SRI) expuesto por Lang et al. (2019) y publicado por el Banco Central Europeo.

Los resultados obtenidos en este documento pueden ser de gran ayuda para los supervisores, dado su enfoque prospectivo que permite la adopción de medidas prudenciales con anticipación.

Como se menciona en Koellner et al. (2005) el análisis tradicional del comportamiento de los fondos de inversión tiene como fundamento la teoría de portafolio de Markowitz (Portfolio Selection: Efficient Diversification of Investments, 1959), en donde su principal objetivo era la clasificación de los fondos de acuerdo con la rentabilidad versus el riesgo de su portafolio o de los mercados donde invierte (Kaiser et al., 2012). Alternativamente, otras medidas tradicionales, tal como Sharpe Ratio, volatilidad de los retornos, etc., son utilizadas para evaluar el desempeño de los portafolios y su óptima selección de activos desde el punto de vista del inversionista y los administradores de los fondos (Hentati et al., 2010).

Ante la relevancia de los fondos de inversión en los mercados financieros y en la captación de recursos del público, su adecuada y oportuna supervisión es cada vez más relevante y ha pasado a estar dentro de las prioridades en la agenda de los diferentes supervisores y reguladores del mercado. Teniendo en cuenta los incentivos de los inversionistas, es evidente que el análisis desde el punto de vista 
del supervisor debe tener un enfoque distinto al realizado por dichos agentes. Es aquí donde el nuevo enfoque de los estudios de las vulnerabilidades de los fondos de inversión cobra relevancia por encina del enfoque en la rentabilidad.

La perspectiva en la cual basan su análisis las calificadoras de riesgo puede ser considerada como la más cercana a las necesidades de los supervisores (Investment Management Quality Ratings, 2020). Sin embargo, muchos de los criterios en los que se basan dichas metodologías siguen sometidos a aspectos cualitativos, en línea con los estándares de supervisión y mejores prácticas dictados por organismos como la International Organization of Securities Commissions (IOSCO) y el Financial Stability Board (FSB).

Teniendo en cuenta lo anterior, una línea de investigación con mayores componentes cuantitativos y alineada con las necesidades de los supervisores respecto a la cuantificación de riesgos e identificación de vulnerabilidades de los fondos de inversión, se basa en la evaluación del efecto de variables macroeconómicas sobre el comportamiento de dichos fondos. Trabajos como los de Das et al., (2005) y Leyva Rayón (2015) exponen las conexiones entre factores claves de la economía y la evolución de los fondos. Por su parte, otros trabajos han ido más allá de las relaciones mencionadas previamente y han encontrado efectos de carácter sistémico, donde la relación no solo va en una vía (variables macroeconómicas que afectan los fondos), sino que el comportamiento del sector de fondos de inversión puede llegar a afectar la estabilidad de los sistemas financieros y, dependiendo de la magnitud, impactar la economía de un país (Consultative Document [2 Nd] Assessment Methodologies for Identifying NonBank Non-Insurer Global Systemically Important Financial Institutions Proposed High-Level Framework and Specific Methodologies, 2015).

Teniendo en cuenta que la gran mayoría de trabajos de riesgo sistémico se enfocan en el sector bancario, varios estudios de las vulnerabilidades de los fondos de inversión han sentado sus bases en la adaptación de estas metodologías al contexto de la industria de los fondos (Jin y Simone, 2015).

Uno de los trabajos más recientes y de mayor acogida en la identificación de vulnerabilidades y medición de riesgo sistémico del sector bancario es el expuesto por Lang et al. (2019), publicado por el Banco Central Europeo. En este documento, los autores calculan un indicador de riesgo sistémico (d-SRI) para la Unión Europea a partir de la selección de diferentes variables macroeconómicas con alto poder de predicción de crisis o eventos de vulnerabilidad. Cabe recordar que el presente documento pretende replicar dicho indicador y adaptarlo al caso de los FIC en Colombia. 
En Colombia, una de las metodologías de calificación para establecimientos de crédito más reconocida es la realizada por el Fondo de Garantías de Instituciones Financieras (Fogafin) (Galindo Vergara et al., 2020). Dicha metodología, propuesta en 2015 y actualizada en 2019, está basada en el modelo de multiplicadores de la Federal Deposit Insurance Corporation (FDIC) de Estados Unidos (Federal Deposit Insurance Corporation, 2016) y tiene como objetivo mapear la fragilidad de las entidades a partir de la selección de varios indicadores financieros con alto poder predictivo para las diferentes categorías de riesgo especificadas ${ }^{2}$.

\section{Marco teórico}

Como se mencionó en la sección anterior, uno de los trabajos más recientes y de mayor acogida en la identificación de vulnerabilidades y medición de riesgo sistémico del sector bancario es el Occasional Paper Series Anticipating the bust: A new cyclical systemic risk indicator to assess the likelihood and severity of financial crises, publicado por el Banco Central Europeo (Lang et al., 2019).

En este trabajo se expone el cálculo de un indicador de riesgo sistémico para la Unión Europea. La metodología aplicada tiene como punto de partida la estimación de las AUROC (Area Under the Receiver Operating Characteristic), técnica estadística ampliamente utilizada para evaluar la efectividad de las señales proporcionadas por los indicadores de alerta temprana. Dicha técnica ha sido empleada para analizar aspectos referentes a la exactitud de pronósticos en diferentes campos, pasando por la ingeniería hasta la medicina (Swets, 1996). A grandes rasgos, las curvas ROC miden la interacción entre los errores de estimación, a saber, el error de emitir una señal errónea versus el error de no detectar una señal que debería ser detectada.

Sin embargo, para los hacedores de política económica, no siempre los errores mencionados tienen la misma ponderación en la toma de decisiones. Dependiendo del objetivo buscado, es más importante la detección de todos los eventos de interés (con lo cual se corre el riesgo de resquebrajar su credibilidad por la posible emisión de falsas alertas), o viceversa, la conservación de la

2 Las categorías de riesgo del modelo son: capital, activos, gestión, exposición al seguro de depósito, rentabilidad, liquidez y concentración. Para cada categoría se selecciona un máximo de tres indicadores financieros con alto poder predictivo para estimar la fragilidad de los establecimientos de crédito. 
credibilidad a partir del menor número de falsas alertas (con lo cual se corre el riesgo de omitir eventos de interés).

Por tanto, los autores del trabajo acuden al uso de la función de pérdidas propuesta por Alessi y Detken (2011), la cual consiste en $L(\theta)=\theta T 1+(1-\theta)$ $T 2$, en donde $T 1$ corresponde al error de no detectar una señal que debería ser detectada y $T 2$ corresponde al error de emitir una señal errónea; mientras que $\theta$ denota las preferencias de los hacedores de política. A partir de la función de pérdidas se calcula cuál sería la utilidad de los hacedores de política, expresada como $U_{a}=$ utilidad absoluta $=\min \{\theta ; 1-\theta\}-L(\theta)$, la cual se explica a continuación.

Tabla 1: Errores de predicción

\begin{tabular}{|l|l|c|c|}
\cline { 3 - 4 } \multicolumn{2}{c|}{} & \multicolumn{2}{c|}{ Realidad } \\
\cline { 3 - 4 } \multicolumn{2}{c|}{} & Vulnerabilidad & Tranquilidad \\
\hline \multirow{3}{*}{ Predicción } & Vulnerabilidad & A & B \\
\cline { 2 - 4 } & Tranquilidad & C & D \\
\hline
\end{tabular}

Fuente: elaboración propia.

A partir de la tabla 1, se infiere que $T 1$ (error de no detectar una señal que debería ser detectada) es equivalente a $\frac{C}{A+C}$ y $T 2$ (error de emitir una señal errónea) es $\frac{B}{B+D}$. Por consiguiente, la función de pérdidas sería igual a $L(\theta)=\theta \frac{C}{A+C}+(1-\theta) \frac{B}{B+D}$.

La expresión $\min \{\theta ; 1-\theta\}$ representa la pérdida que tendría un hacedor de política si no tiene en cuenta el modelo o no toma alguna decisión. Si $\theta$ es menor de 0,5 (mayor aversión hacia predecir vulnerabilidad en periodos de tranquilidad, i.e. T2: falsos positivos), la solución de esquina, en donde al hacedor de política solo le importa $T 2$, se obtiene ignorando las vulnerabilidades que emite el modelo, es decir, no emitir ninguna predicción de vulnerabilidad, por lo que $A=B=0$, con lo que se obtiene $L(\theta)=\theta$.

Por el contrario, si $\theta$ es mayor de 0,5 (mayor aversión hacia predecir tranquilidad en periodos de vulnerabilidad, i.e. T1: falsos negativos), la solución de esquina, en donde al hacedor de política solo le importa $T 1$, se obtiene 
asumiendo que siempre estamos en periodos de vulnerabilidad, es decir, emitir siempre predicciones de vulnerabilidad, por lo que $C=D=0$, con lo que se obtiene $L(\theta)=1-\theta$.

Si el hacedor de política, al no tener en cuenta el modelo o no tomar alguna decisión, obtiene una pérdida de $\min \{\theta ; 1-\theta\}$, un modelo que implique una pérdida menor a este monto representa que es útil para un $\theta$ dado. Por tanto, la utilidad de un modelo se obtiene a partir de la expresión, $U_{a}=\min \{\theta ; 1-\theta\}-$ $L(\theta)$, la cual expone el monto de pérdida que el hacedor de política se ahorró o redujo al utilizar el modelo con sus preferencias $(\theta)$.

Con el fin de que la utilidad no esté sesgada al alza o a la baja, evitando la adecuada comparabilidad de los indicadores, los autores presentan la utilidad en términos relativos tal como lo propone Sarlin (2013), $U_{r}=$ utlidad relativa $=$ $U_{a} / \min \{\theta ; 1-\theta\}$. De esta manera, la utilidad de un indicador muestra el porcentaje de pérdida que el hacedor de política se ahorró al utilizar el modelo con sus preferencias $(\theta)$. Por tanto, a mayor utilidad relativa $\left(U_{r}\right)$ mayores beneficios de alerta temprana tendrá el indicador.

La función de utilidad relativa se calcula para cada uno de los indicadores candidatos a ser parte del modelo de alerta temprana y, en conjunto con las AUROC, se obtiene un coeficiente compuesto que mide el poder de predicción, expresado como Coeficiente compuesto $=\frac{2}{3}$ AUROC $+\frac{1}{3}$ utilidad relativa . La escogencia de un coeficiente compuesto tiene como fundamento incluir un parámetro globalmente aceptado para la detección de alertas tempanas como las AUROC, junto con un elemento que incorpore las preferencias de los hacedores de política como lo es la medida de utilidad. Dado el grado de subjetividad que trae consigo la medida de utilidad, la mayor ponderación en el coeficiente compuesto se le asigna a las AUROC (2/3).

El coeficiente compuesto es aplicado a indicadores financieros y económicos con amplio reconocimiento en la literatura por su poder de predicción de escenarios de vulnerabilidad. En la implementación de esta metodología en la detección de fragilidades de los FIC es necesario evaluar variables propias de dichos fondos, tanto de los estados financieros como de elementos adicionales relacionados con su liquidez, rentabilidad y la composición de sus portafolios de inversión. A su vez, estas variables deben ser complementadas con indicadores macroeconómicos que evidencien el comportamiento de la liquidez en el sistema financiero, así como periodos de inestabilidad en la economía. 
Cabe aclarar que el cálculo del coeficiente compuesto no se realiza sobre los indicadores puros, sino que se aplica sobre sus variaciones trimestrales, anuales, bianuales, trianuales y su diferencia frente a la tendencia de largo plazo, obtenida a partir del filtro de Hodrick-Prescott.

Los indicadores deben ser agrupados en categorías que recojan características similares, por ejemplo, los autores seleccionan las categorías de riesgo sistémico definidas por el Consejo Europeo de Riesgo Sistémico (ESRB), a saber: sobrevaloración de precios de vivienda, carga de deuda del sector privado, profundización de crédito, subvaloración de precios de activos financieros (mercado accionario) y desbalances externos. En el caso de la aplicación a los FIC, las categorías serían las siguientes: estructura de balance, liquidez y rentabilidad de los FIC; mercados financieros; dinero circulante en la economía (liquidez de la economía), y desbalances macroeconómicos. Por cada categoría se seleccionan los indicadores con mayor poder de predicción, calculados a partir del coeficiente compuesto.

Posteriormente, los indicadores seleccionados son normalizados restando la media $^{3}$ y dividiendo por la desviación estándar, para garantizar la comparabilidad. Finalmente, a partir de una regresión de mínimos cuadrados ordinarios (MCO), se obtienen las ponderaciones de cada uno de los indicadores seleccionados, restringiendo los coeficientes para que sean positivos, mayores al $5 \%, \mathrm{y}$ que la sumatoria de estos sea igual a $100 \%$, con el fin de construir el indicador de alerta temprana para la detección de vulnerabilidades.

\section{Construcción del indicador de alerta temprana para los FIC}

De acuerdo con la metodología utilizada por Lang et al. (2019), en el d-SRI del Banco Central Europeo, para la construcción del indicador de alertas tempranas se deben tener en cuenta cinco etapas: identificación de periodos de vulnerabilidad, selección de categorías de riesgo, selección de indicadores por categoría, cálculo de las ponderaciones de cada categoría y cálculo del indicador de alerta temprana.

A continuación, se expone la adaptación de las tres primeras etapas para el caso de un indicador de alerta temprana para la identificación de vulnerabilidades en la industria de los FIC. Las últimas dos etapas se presentan en el apartado 3.

3 En el documento de referencia, los autores realizan la normalización de los indicadores a partir de la mediana en lugar de la media, debido a que la muestra de datos no era de un solo país sino de todos los países de la Zona Euro, junto con Dinamarca, Suecia y Reino Unido. 


\subsection{Identificación de periodos de vulnerabilidad en los FIC}

A partir de la variación trimestral del valor neto de los fondos de inversión colectiva (VNF) es posible evidenciar cuatro episodios de vulnerabilidad entre 2008 y el segundo trimestre de 2020.

Figura 1: Variación trimestral crecimiento de activos FIC

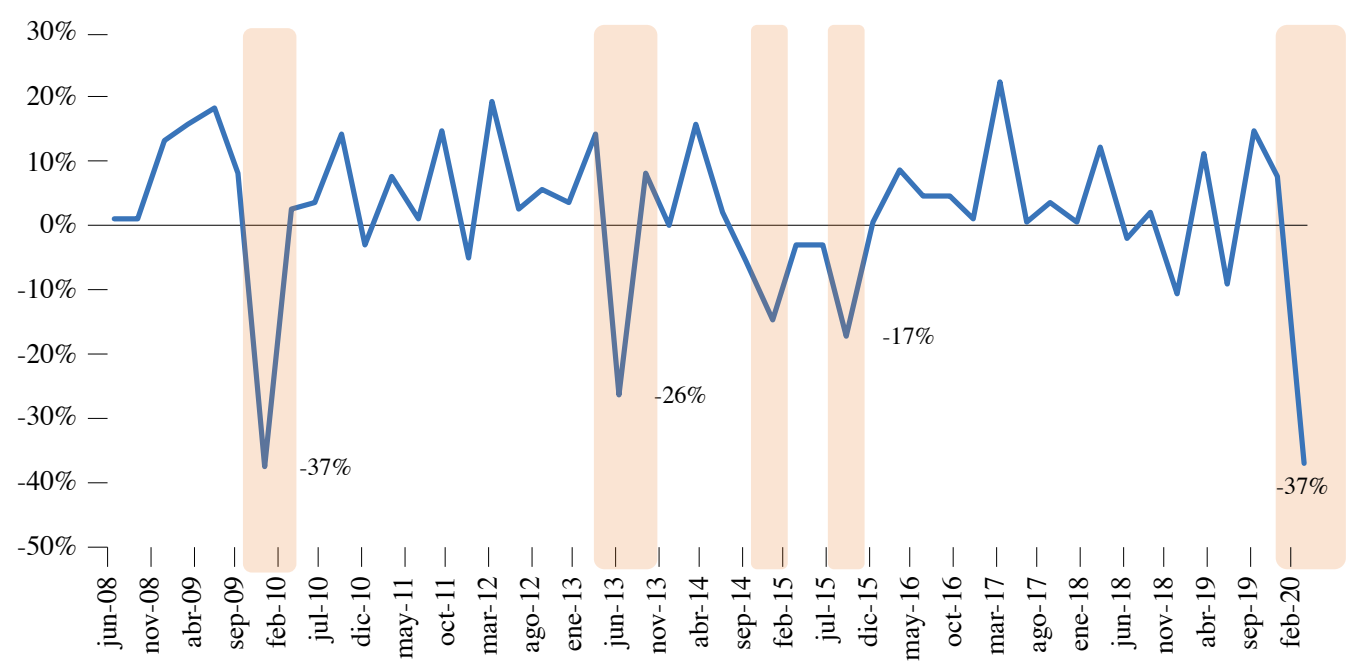

Fuente: Federación Iberoamericana de Fondos de Inversión (FIAFIN). Cálculos propios.

Como se muestra en la figura 1, el primer episodio de vulnerabilidad se presentó en el cuarto trimestre de 2009 y corresponde al rezago de la crisis financiera internacional de 2008-2009; el segundo, entre el segundo y el tercer trimestre de 2013, fue ocasionado por los efectos en los mercados financieros de la crisis de deuda europea y el anuncio del incremento de tasas de interés en Estados Unidos, junto con el final de los programas de inyección masiva de liquidez a la economía (tapering). En el tercer episodio, en el cuarto trimestre de 2014 y el tercer trimestre de 2015, se vieron los mayores impactos a los mercados tras el fin del superciclo de los commodities, que en Colombia, a raíz de la drástica caída de los precios del petróleo, afecto fuertemente los sectores real y financiero. Finalmente, entre finales del primer trimestre y principios del segundo trimestre de 2020, como consecuencia de los efectos ocasionados en la economía y los mercados financieros por la declaración de pandemia del covid-19. 
Una vez identificados los periodos de vulnerabilidad, se genera una variable indicadora, donde se marcan con 1 los 6 meses anteriores al periodo de vulnerabilidad y con 0 los demás meses (incluido el periodo de vulnerabilidad). Esta variable binomial corresponde a la variable dependiente del modelo para el cálculo del indicador de vulnerabilidad. La acción de marcar con 1 los meses anteriores a los hechos de fragilidad responde a la necesidad de generar un indicador predictivo y no uno contemporáneo (figura 2).

Figura 2: Variable de vulnerabilidad (variable dependiente)

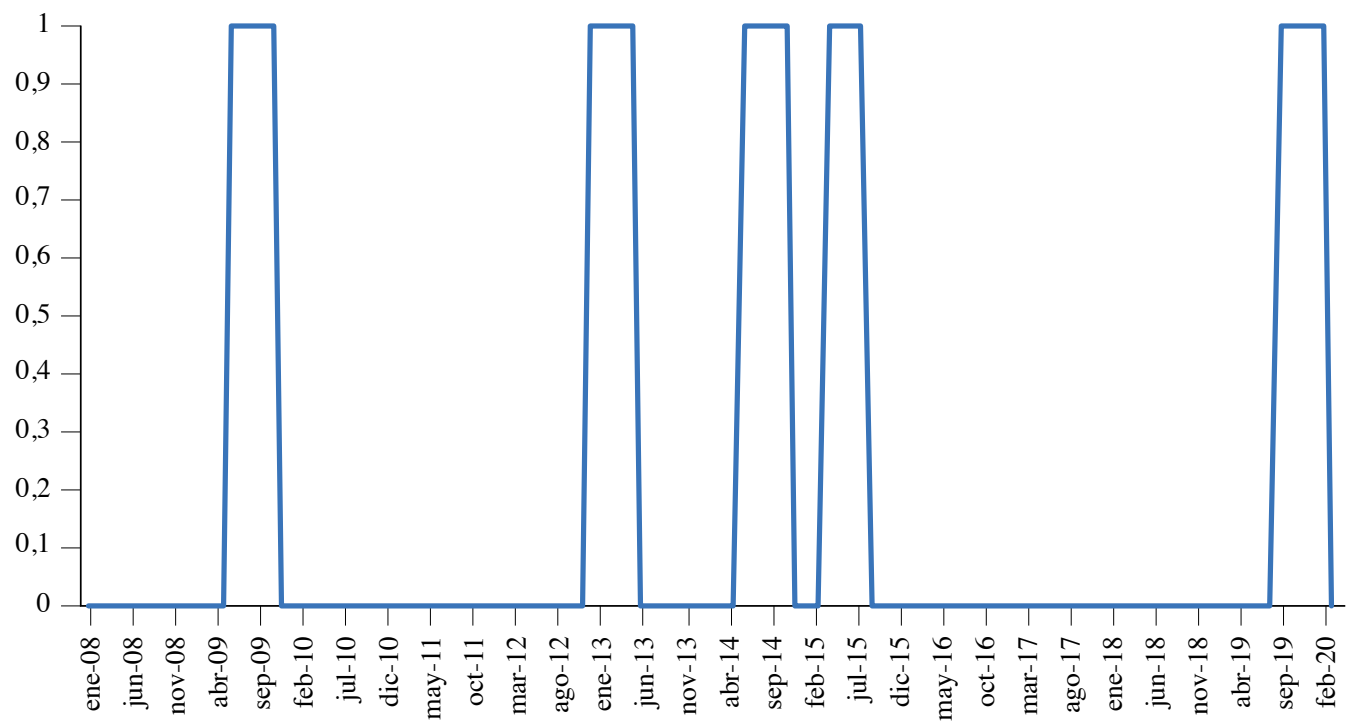

Fuente: Federación Iberoamericana de Fondos de Inversión (FIAFIN). Cálculos propios.

\subsection{Selección de categorías e indicadores de riesgo (variables independientes)}

Dada la naturaleza de diversificación de negocios de los FIC se encuentra una amplia gama de factores que pueden afectar su desempeño. Cabe recordar que, a partir de las inversiones que realizan, los FIC se categorizan como fondos de inversión colectiva de tipo general, fondos de inversión colectiva del mercado monetario, fondos de inversión colectiva inmobiliarios, fondos de inversión colectiva bursátiles y fondos de capital privado.

A su vez, de acuerdo con la modalidad de redención de los aportes a los FIC, estos se pueden clasificar en abiertos sin pacto de permanencia (los inversionistas 
pueden disponer de sus recursos sin penalidad cuando lo deseen), abiertos con pacto de permanencia (los fondos cuentan con ventanas de salida para que los inversionistas dispongan de sus recursos, una penalidad será cobrada en cualquier otro momento) y fondos cerrados (no se permite disponer de los recursos hasta el plazo estipulado en la creación del fondo). El presente trabajo se enfoca en los FIC abiertos sin pacto de permanencia (ASPP) debido a que son los de mayor exposición a fluctuaciones del mercado y donde se encuentra el mayor número de inversionistas.

Los factores que pueden afectar el desempeño de los FIC ASPP se pueden clasificar en seis grandes categorías: estructura de balance de los FIC, liquidez de los FIC, rentabilidad de los FIC, mercados financieros, dinero circulante en la economía (liquidez de la economía) y desbalances macroeconómicos.

La primera categoría, estructura de balance de los FIC, incluye rubros de los estados financieros de los FIC relevantes (diferentes a cuentas relacionadas con el VNF ya que es tenido en cuenta en la variable dependiente), tales como money flow (aportes y retiros netos) y el portafolio de inversiones. La segunda categoría, liquidez de los FIC ${ }^{4}$ tiene en cuenta el efectivo, los activos líquidos de alta calidad (ALAC) y el máximo requerimiento de liquidez (MRLQ) ${ }^{5}$. La tercera categoría de rentabilidad de los FIC incluye la cuenta de estados financieros, rendimientos abonados (utilidad), la rentabilidad mensual, semestral y anual. La fuente de información para las tres categorías enunciadas son los estados financieros de los FIC y el formato 523, "Informe Rentabilidades Fondos de Inversión Colectiva" de la Superintendencia Financiera de Colombia ${ }^{6}$.

La cuarta categoría, mercados financieros, abarca los indicadores de referencia de los principales activos financieros que afectan los FIC, tales como, COLCAP, tasa de captación de los CDT, tasa de cambio TRM, precio del Brent, tasa cero cupón en pesos de los TES a 1, 5 y 10 años. La quinta categoría, dinero

4 En el anexo 1 se muestra el tratamiento de datos que se realizó a cada una de las variables evaluadas en el modelo.

5 Para profundizar en la definición y composición de los indicadores de liquidez de los FIC consultar el Anexo 3 del Capítulo vi de la Circular Básica Contable y Financiera (Circular Externa 100 de 1995), en https://www.superfinanciera.gov.co/descargas?com=institucional\& name $=$ pubFile1012099 $\&$ downloadname $=$ cap06anexo3.docx

6 La información mencionada de los FIC puede ser consultada en la página web de la Superintendencia Financiera de Colombia, en https://www.superfinanciera.gov.co/ReporteInformacionfIC/

ODEON, ISSN: 1794-1113, E-ISSN: 2346-2140, № 19, julio-diciembre de 2020, pp. 31-79 
circulante en la economía, está compuesta por los depósitos de los establecimientos de crédito (EC) ${ }^{7}$, el M3 y la base monetaria. La selección de los depósitos de los EC como posible indicador de alerta temprana de los FIC es consecuencia de su naturaleza de refugio en tiempos de alta volatilidad en los mercados, en donde los inversionistas institucionales tienden a retirar recursos de los FIC y depositarlos en cuentas vista de las entidades bancarias. A su vez, las personas naturales con inversiones en fondos tienen incentivos para mover los recursos de los FIC hacia cuentas vista dado que estas están amparadas por el seguro de depósito de Fogafin que cubre hasta \$50 millones en caso de liquidación del EC y no tiene cobertura para las inversiones en FIC.

Finalmente, la sexta categoría, desbalances macroeconómicos, incluye el indicador de seguimiento a la economía (ISE) del DANE y la tasa de desempleo. La fuente de información para las tres últimas categorías corresponde al Banco de la República de Colombia y al DANE.

A cada variable se le calcula su crecimiento (o diferencia, dependiendo de su naturaleza) mensual, bimensual, trimestral, semestral, anual, bianual y trianual. A su vez, se realiza el cálculo de las series extrayendo la tendencia a partir del filtro de Hodrick-Prescott con multiplicadores de 26.000, 400.000 y $13.322 .500^{8}$.

\subsection{Selección de indicadores por categoría}

En línea con lo expuesto por Lang et al.(2019), una vez definidas las categorías de riesgo es necesario seleccionar los indicadores con mayor poder de predicción en cada una de las categorías. Para tal efecto, los autores proponen el cálculo de un coeficiente compuesto el cual incorpora una medida ampliamente aceptada para la detección de alertas tempanas como lo son las AUROC, junto con un elemento con cierto componente subjetivo (utilidad relativa) con el fin de tener en cuenta las preferencias de los hacedores de política.

Cabe mencionar que tanto el análisis de las AUROC como el cálculo de la utilidad relativa tienen como punto de partida las mediciones de los errores tipo I y tipo II, los cuales son empleados con el fin de reflejar los costos en los que

7 Depósitos en cuenta corriente + Cuentas de ahorro + CDT + Bonos + Otros depósitos a la vista + Repos + Depósitos Fiduciarios. Datos tomados de los agregados monetarios y crediticios del Banco de la República.

8 Correspondiente al ajuste de lambda propuesto por Backus y Kehoe (1992), i.e. lambda para periodicidad anual $*$ periodos en un año ${ }^{\wedge}$, que para el caso de este documento es $100 * 365^{\wedge} 2$. 
incurren los hacedores de política en la toma de decisiones. Como antecedente del uso de los errores de estimación para la aproximación de la función de pérdidas se encuentra el trabajo de Demirgüç-Kunt y Detragiache (2000), quienes introdujeron la noción de la función de pérdida de un hacedor de política en términos de los costos por las acciones preventivas y los errores de tipo I y tipo II en un contexto de crisis bancaria.

En los años siguientes al trabajo mencionado, este tipo de aproximación a la función de pérdida se adaptó para otros tipos de crisis, por ejemplo, crisis de deuda (Fuertes y Kalotychou, 2007), crisis de monedas (Bussière y Fratzscher, 2008) y auges de los precios de los activos (Alessi y Detken, 2011), lo que simplificó el cálculo de la función de pérdida al dejar de tener en cuenta los costos de las acciones preventivas y centrarse principalmente en el equilibrio entre los errores de tipo I y tipo II. Esta clase de enfoque elimina la dificultad presente a la hora de estimar los costos ciertos de las acciones preventivas que toman los hacedores de política con el fin de mitigar los efectos de las crisis.

A continuación, se presenta el análisis y cálculo de las AUROC, la utilidad relativa y el coeficiente compuesto, los cuales tienen como base la estimación de los errores tipo I y tipo II.

\subsubsection{AUROC}

La selección de variables que forman parte de los modelos de alerta temprana se puede realizar de diversas maneras, desde estimaciones multivariadas como los modelos de vectores autorregresivos (VAR) hasta medidas enfocadas en los errores de predicción como las AUROC. Esta última metodología ha sido ampliamente utilizada en los modelos de alertas tempranas debido a sus ventajas, principalmente, la sencilla implementación computacional, la facilidad de interpretación y la comparabilidad entre variables.

A grandes rasgos, las curvas ROC miden la interacción entre los errores de estimación, a saber, el error de emitir una señal errónea versus el error de no detectar una señal que debería ser detectada. Asimismo, un indicador con AUROC igual a 1 posee mayores propiedades de alerta temprana que uno con AUROC igual a 0,8 . Por su parte, un indicador con AUROC menor o igual a 0,5 no cuenta con propiedades adecuadas para ser considerado un buen predictor de alerta temprana. Por ejemplo, en la figura 3 se evidencia que la variable crecimiento anual del Brent, con un AUROC de 0,73 , posee un mayor poder de predicción y es un mejor 
candidato para formar parte del modelo en comparación con el crecimiento anual del M3, que cuenta con un AUROC de 0,60.

Figura 3: Curvas ROC
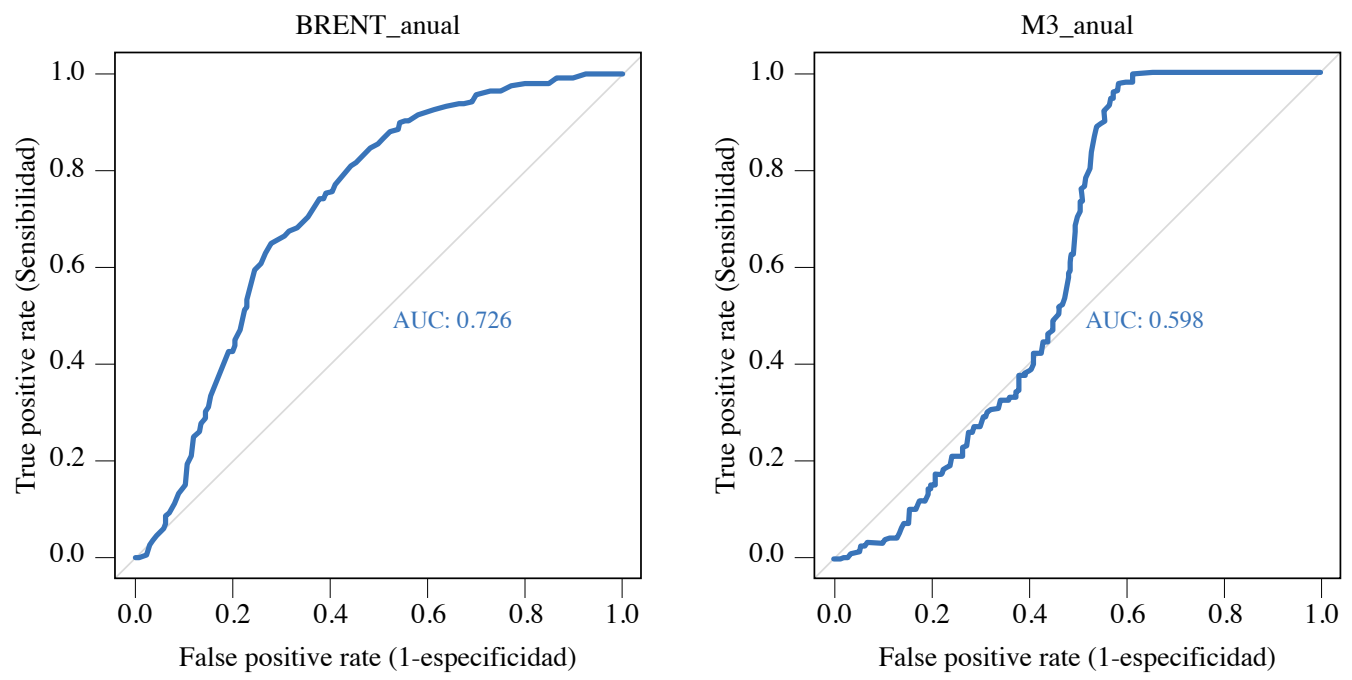

Fuente: cálculos propios.

Para el caso del modelo de alerta temprana en los FIC, el cálculo de las AUROC se realiza comparando la variable indicadora de vulnerabilidad (variable dependiente del modelo) contra cada una de las transformaciones ${ }^{9}$ de los indicadores candidatos a formar parte del modelo (variables independientes). Tal como se muestra en la tabla 2, el indicador con mayor coeficiente AUROC pertenece a la categoría de Mercados Financieros y corresponde a la variación anual del Brent con 0,73 . Sin embargo, la categoría que más se destaca por sus altas AUROC es liquidez de los FIC, ya que de los 10 indicadores con mayor AUROC ${ }^{10}, 4$ pertenecen a esta categoría.

9 Crecimiento (o diferencia, dependiendo de su naturaleza) mensual, bimensual, trimestral, semestral, anual, bianual y trianual. A su vez, se realiza el cálculo de las series extrayendo la tendencia a partir del filtro de Hodrick-Prescott con multiplicadores de 26.000 (HP26.000), 400.000 (HP400.000) y 13’322.500 (HP _ RT), correspondientes al ajuste de lambda propuesto por Backus y Kehoe (1992) (i.e. lambda para periodicidad anual * periodos en un año ${ }^{\wedge}$, que para el caso de este documento es $\left.100 * 365^{\wedge} 2\right)$.

10 En el anexo 3 se encuentran los resultados de las AUROC para todas las variables evaluadas. 
Tabla 2: Indicadores con mayor AUROC

\begin{tabular}{|l|l|}
\hline \multicolumn{1}{|c|}{ Indicador } & AUROC \\
\hline BRENT_anual & 0,726 \\
\hline ALAC_trianual & 0,711 \\
\hline EFECTIVO_trianual & 0,697 \\
\hline TES_5_YEARS_anual & 0,694 \\
\hline COLCAP_HP_RT & 0,688 \\
\hline MRLQ_trianual & 0,687 \\
\hline TES_10_YEARS_anual & 0,679 \\
\hline BRENT_HP_RT & 0,675 \\
\hline TRM_HP _ RT & 0,673 \\
\hline EFECTIVO_bianual & 0,663 \\
\hline
\end{tabular}

Fuente: cálculos propios.

\subsubsection{Utilidad}

En conjunto con el cálculo de las AUROC, la selección de indicadores que forman parte del modelo estimado por Lang et al. (2019) se realiza con base en una función de utilidad que tiene en cuenta las preferencias de los hacedores de política entre obtener menores tasas de falsos negativos (vulnerabilidades no detectadas) $\mathrm{u}$ obtener menores tasas de falsos positivos (falsas alarmas de vulnerabilidad).

La importancia de la implementación de una función de utilidad que refleje las preferencias de los hacedores de política radica en el objetivo que se busca alcanzar. Si el mayor interés del hacedor de política se enfoca en resguardar su credibilidad, le dará mayor importancia a minimizar los falsos positivos; por el contrario, si su objetivo radica en la detección de todos los eventos de interés, corriendo el riesgo de resquebrajar su credibilidad por la posible emisión de falsas alertas, el hacedor de política se enfocará en mayor medida en minimizar los falsos negativos.

Por tanto, el cálculo de la función de utilidad de los hacedores de política tiene como punto de partida la medición de una función de pérdidas $L(\theta)$ a partir de la interacción entre los dos errores de estimación: 


$$
L(\theta)=\theta T 1+(1-\theta) T 2
$$

Donde $T 1$ corresponde al error de no detectar una vulnerabilidad cuando en realidad ocurrió (falsos negativos), $T 2$ corresponde al error de detectar una vulnerabilidad cuando en realidad es un periodo de tranquilidad (falsos positivos) y $\theta$ refleja la aversión de los hacedores de política a emitir falsos negativos versus falsos positivos. Un $\theta>0,5$ indica que el hacedor de política está más preocupado por detectar todos los eventos de interés por encima de la conservación de su credibilidad.

El hecho de expresar la función de pérdidas en términos de la ponderación con las preferencias de los hacedores de política $(\theta)$ permite tener una aproximación a los "costos" que, de otra manera, serían complejos de estimar ante la incertidumbre que generan los efectos de las acciones preventivas, las falsas alarmas y las vulnerabilidades no detectadas.

Por consiguiente, si el hacedor de política no utiliza las señales de vulnerabilidad que le provee el modelo y no toma acciones al respecto, tendría una pérdida entendida como $^{11}$ :

$$
\min \{\theta ; 1-\theta\}
$$

Usando las ecuaciones (1) y (2) se define la medida de "utilidad" de un modelo o decisión de política como la diferencia entre la pérdida de ignorar el modelo, i.e. la ecuación (2); y la pérdida asociada a la implementación del modelo, i.e. la ecuación (1).

$$
U_{a}=\min \{\theta ; 1-\theta\}-L(\theta)
$$

Donde: $U_{a}$ es la utilidad expresada en términos absolutos obtenida por los hacedores de política, que evidencia el menor costo en que incurrieron dada la utilización del modelo estimado.

Sin embargo, con el fin de obtener medidas comparables de utilidad entre los distintos indicadores candidatos a formar parte del modelo de alertas tempranas, la utilidad debe ser expresada en término relativos, dividiendo la utilidad absoluta por la ecuación (2):

11 La interpretación de la ecuación (2) fue explica en el apartado 1, Marco teórico. 


$$
U_{r}=U_{a} / \min \{\theta ; 1-\theta\}
$$

La utilidad relativa $\left(U_{r}\right)$ expresa la proporción del costo inicial (i.e. la ecuación [2]) que los hacedores de política ahorraron o disminuyeron al utilizar el modelo con sus preferencias $(\theta)$. A mayor utilidad relativa mayores beneficios de alerta temprana tendrá el indicador.

En la figura 4 se presenta un ejemplo del comportamiento de la utilidad relativa calculada para el crecimiento trianual de los activos líquidos de alta calidad (ALAC) de los FIC, perteneciente a la categoría de liquidez FIC.

Figura 4: Función de utilidad relativa ALAC

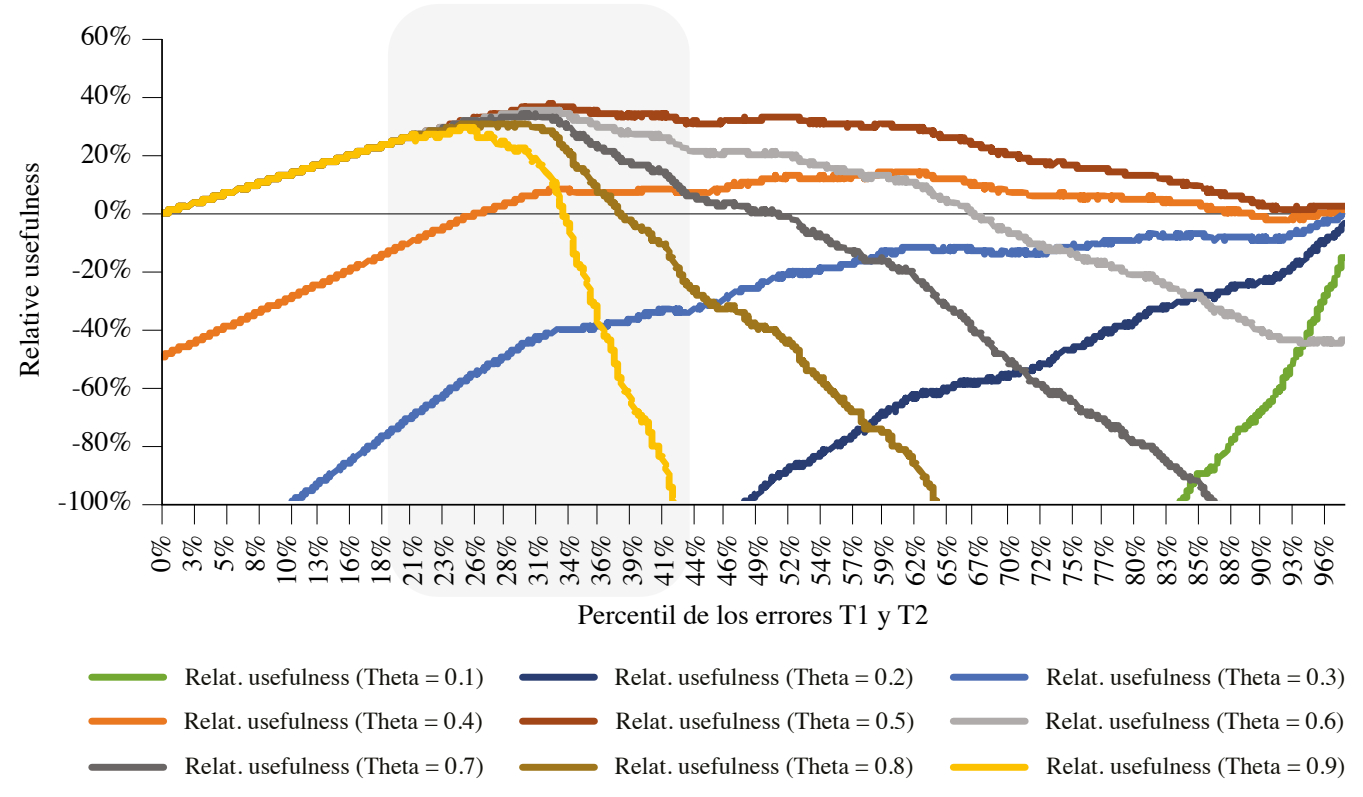

Fuente: cálculos propios.

Cada punto de las curvas ROC refleja la combinación de falsos positivos (T2) y falsos negativos (T1) para un percentil dado de la distribución de cada indicador. En línea con lo anterior, con cada punto de la curva ALAC se estima la función de pérdidas expuesta en la ecuación (1), teniendo en cuenta diferentes valores de las preferencias de los hacedores de política $(\theta)$. Para el caso del crecimiento trianual de los ALAC, los hacedores de política obtendrían utilidad $(\theta>0)$ siempre y cuando $\theta$ sea mayor o igual a 0,4 . 
Al comparar los resultados se evidencia que la mayor utilidad relativa $\left(U_{r}\right)$ se encuentra cuando $\theta$ es igual a 0,5 , con lo que se alcanza un "ahorro" del $37,8 \%$ del costo inicial, lo cual se obtiene con una tasa de falsos positivos (T2) de $59,2 \%$ y de falsos negativos (T1) de $3 \%$, equivalentes al percentil 32 de la distribución del indicador.

Sin embargo, las preferencias balanceadas $(\theta=0,5)$ no reflejan necesariamente los intereses del hacedor de política. Suponiendo que se tenga un interés ligeramente mayor en detectar el mayor número de vulnerabilidades frente a emitir falsos positivos, sus preferencias se ven reflejadas en un $\theta$ levemente mayor a 0,5 . Por ejemplo, si su preferencia es de $\theta=0,6$, la utilidad continúa maximizándose en el percentil 32 de la distribución del indicador equivalente a un $T 2$ de $59,2 \%$ y un $T 1$ de $3 \%$, arrojando una $U_{r}$ de $36,3 \%$ del costo inicial.

Ahora bien, si los intereses del hacedor de política se enfocan en obtener el menor número de vulnerabilidades no detectadas (falsos negativos) por encima de resquebrajar su credibilidad, sus preferencias se verían reflejadas en un $\theta$ significativamente mayor a 0,5 . Por ejemplo, si su preferencia es de $\theta=0,8$, la utilidad se maximiza en el percentil 25 de la distribución del indicador, equivalente a una tasa de falsos positivos (T2) de 67,1\% y de falsos negativos (T1) de $0,29 \%$, generando un ahorro del $31,7 \%$ del costo inicial $\left(U_{r}\right)$.

Siguiendo con el ejemplo del crecimiento trianual de los ALAC, en la figura 5 se presenta la tasa óptima de falsos positivos y falsos negativos con la cual se maximiza la utilidad para cada nivel de preferencias $(\theta)$ de los hacedores de política. La selección de un $\theta \geq 50 \%$ implica una tasa de errores tipo II (falsos positivos) entre 59 y $73 \%$, y una tasa de errores tipo I (falsos negativos) entre 3 y $0,3 \%$.

En resumen, para el caso del crecimiento trianual de los ALAC, indicador que presenta la segunda mayor AUROC con 0,71 , la mayor utilidad relativa $\left(U_{r}\right)$ se obtiene cuando $\theta=50 \%$, lo que refleja el mayor ahorro posible del costo inicial. Sin embargo, con diferentes niveles de preferencias el hacedor de política puede obtener utilidades relativamente similares y con menores tasas de errores que reflejen de una mejor manera su objetivo de política (detectar un mayor número de vulnerabilidades o emitir el menor número de vulnerabilidades erróneas).

En la tabla 3 se exponen los 12 indicadores con mayor utilidad relativa ${ }^{12}$. Se destaca que no necesariamente el indicador con mayor AUROC (crecimiento

12 En el anexo 3 se encuentran los resultados de la utilidad relativa para todas las variables evaluadas. 
anual del Brent), presenta la mayor utilidad relativa. A su vez, la mayoría de indicadores obtiene una mayor utilidad con un $\theta=0,5$, aunque se puede dar el caso de preferencias no balanceadas con las cuales se obtiene la mayor utilidad, como sucede para el indicador de crecimiento trianual de los rendimientos abonados anualizados (utilidad anualizada) de los FIC, donde la mayor utilidad se obtiene $\operatorname{con} \theta=0,86$.

Figura 5: Errores óptimos $T 1$ (FN) y $T 2$ (FP). Crecimiento Trianual ALAC

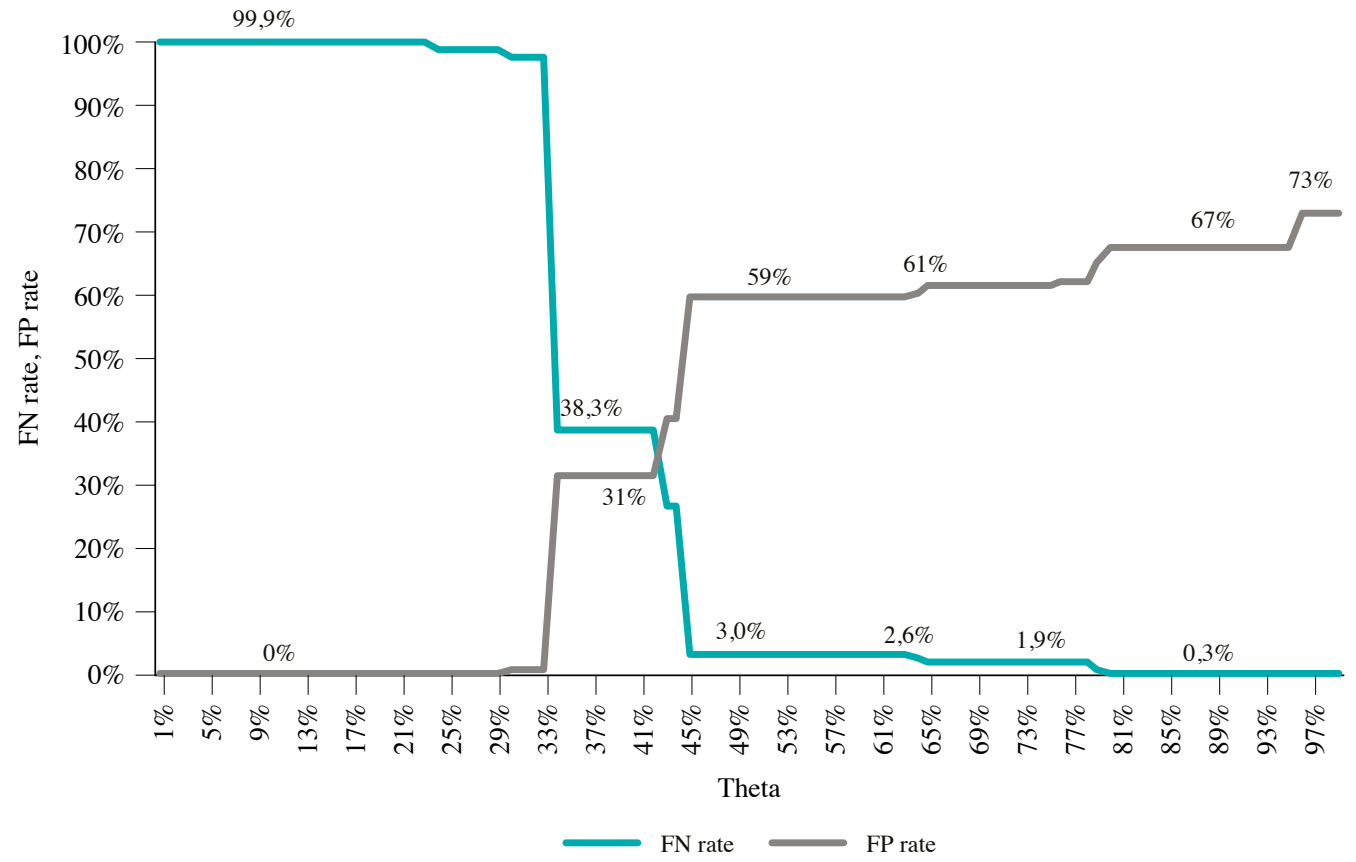

Fuente: cálculos propios.

Tabla 3: Indicadores con mayor utilidad relativa $\left(U_{r}\right)$

\begin{tabular}{|l|c|c|c|c|c|}
\hline \multicolumn{1}{|c|}{ Indicador } & $\theta$ óptimo & $\begin{array}{c}\text { Percentil } \\
\text { óptimo }\end{array}$ & $\begin{array}{c}\text { Tasa falsos } \\
\text { negativos }\end{array}$ & $\begin{array}{c}\text { Tasa falsos } \\
\text { positivos }\end{array}$ & $\begin{array}{c}\text { Utilidad } \\
\text { relativa }\end{array}$ \\
\hline M3_trianual & 0,500 & 0,673 & 0,309 & 0,235 & 0,457 \\
\hline BRENT_bianual & 0,500 & 0,368 & 0,017 & 0,548 & 0,435 \\
\hline Rent_semestral_anual & 0,500 & 0,533 & 0,191 & 0,382 & 0,427 \\
\hline ISE_bianual & 0,500 & 0,384 & 0,040 & 0,538 & 0,422 \\
\hline
\end{tabular}




\begin{tabular}{|l|c|c|c|c|c|}
\hline \multicolumn{1}{|c|}{ Indicador } & $\theta$ óptimo & $\begin{array}{c}\text { Percentil } \\
\text { optimo }\end{array}$ & $\begin{array}{c}\text { Tasa falsos } \\
\text { negativos }\end{array}$ & $\begin{array}{c}\text { Tasa falsos } \\
\text { positivos }\end{array}$ & $\begin{array}{c}\text { Utilidad } \\
\text { relativa }\end{array}$ \\
\hline COLCAP_bianual & 0,500 & 0,377 & 0,037 & 0,546 & 0,417 \\
\hline Depositos_trianual & 0,500 & 0,630 & 0,299 & 0,288 & 0,413 \\
\hline Rent_anual_anual & 0,500 & 0,378 & 0,049 & 0,541 & 0,411 \\
\hline M3_anual & 0,500 & 0,329 & 0,025 & 0,588 & 0,387 \\
\hline ALAC_trianual & 0,500 & 0,319 & 0,030 & 0,592 & 0,378 \\
\hline BRENT_anual & 0,500 & 0,646 & 0,351 & 0,275 & 0,373 \\
\hline TES_1_YEAR_trianual & 0,500 & 0,372 & 0,083 & 0,555 & 0,363 \\
\hline $\begin{array}{l}\text { Rendim_abon_anual_ } \\
\text { trianual }\end{array}$ & 0,860 & 0,282 & - & 0,638 & 0,362 \\
\hline
\end{tabular}

Fuente: cálculos propios.

Los indicadores con mayor ahorro del costo inicial, entendido como la utilidad relativa $\left(U_{r}\right)$, son el crecimiento trianual del M3 y el crecimiento bianual del Brent, con un ahorro de 46 y $44 \%$ del costo inicial, respectivamente.

\subsubsection{Coeficiente compuesto}

Una vez calculada el área bajo la curva (AUROC) y la utilidad relativa $\left(U_{r}\right)$ para cada uno de los indicadores candidatos a ser parte del modelo, se estima el coeficiente compuesto para cada indicador a partir de la siguiente ecuación:

$$
\text { Coef.compuesto }=2 / 3 \text { AUROC }+1 / 3 U_{r}
$$

La intuición detrás del cálculo del coeficiente compuesto radica en incluir un parámetro altamente reconocido en los modelos de alertas tempanas como son las AUROC, en conjunto con un elemento que tiene en cuenta las preferencias de los hacedores de política junto con los costos de sus decisiones, reflejado en la utilidad relativa. En cuanto a la ponderación de cada uno de estos dos componentes de la ecuación (5), Lang et al. (2019) argumentan que, dado el grado de subjetividad que trae consigo la medida de utilidad relativa, el mayor peso en el coeficiente compuesto se le asigna a las AUROC (2/3). 
Tabla 4: Indicadores con mayor coeficiente compuesto

\begin{tabular}{|l|c|c|c|}
\hline \multicolumn{1}{|c|}{ Indicador } & AUROC & Utilidad relativa & Coeficiente compuesto \\
\hline BRENT_anual & 0,726 & 0,373 & 0,608 \\
\hline ALAC_trianual & 0,711 & 0,378 & 0,600 \\
\hline M3_trianual & 0,659 & 0,457 & 0,592 \\
\hline EFECTIVO_trianual & 0,697 & 0,339 & 0,578 \\
\hline BRENT_bianual & 0,645 & 0,435 & 0,575 \\
\hline COLCAP_bianual & 0,652 & 0,417 & 0,574 \\
\hline ISE_bianual & 0,645 & 0,422 & 0,570 \\
\hline Rent_semestral_anual & 0,639 & 0,427 & 0,568 \\
\hline TES_5_YEARS_anual & 0,694 & 0,310 & 0,566 \\
\hline Depositos_trianual & 0,638 & 0,413 & 0,563 \\
\hline MRLQ_trianual & 0,687 & 0,301 & 0,559 \\
\hline MRLQ_anual & 0,655 & 0,361 & 0,557 \\
\hline Rent_anual_anual & 0,627 & 0,411 & 0,555 \\
\hline TES_10_YEARS_anual & 0,679 & 0,298 & 0,552 \\
\hline Rendim_abon_anual_trianual & 0,644 & 0,362 & 0,550 \\
\hline COLCAP_HP_RT & 0,688 & 0,275 & 0,550 \\
\hline BRENT_HP_RT & 0,675 & 0,279 & 0,543 \\
\hline Rent_semestral_bim & 0,649 & 0,322 & 0,540 \\
\hline TRM_HP_RT & 0,673 & 0,265 & 0,537 \\
\hline Rent_semestral_men & 0,656 & 0,291 & 0,279 \\
\hline APORT_RET_anual_anual & 0,659 & 0,534 \\
\hline
\end{tabular}

Fuente: cálculos propios.

Los indicadores que forman parte del indicador de alertas tempranas de los FIC corresponden a los que presentan un mayor coeficiente compuesto para cada una de las categorías de análisis mencionadas en la sección 3.2. 
De acuerdo con la tabla 4, la cual expone los indicadores con mayor coeficiente compuesto ${ }^{13}$, las variables seleccionadas por cada categoría son: crecimiento anual del Brent para la categoría de mercados financieros, crecimiento trianual de los ALAC en la categoría de liquidez de los FIC, crecimiento trianual del M3 para la categoría dinero circulante en la economía, crecimiento bianual del ISE para la categoría desbalances macroeconómicos, cambio anual de la rentabilidad semestral de los FIC en la categoría rentabilidad FIC y la variación anual de money flow (acumulado de 12 meses de los aportes y retiros netos) de los FIC para la categoría estructura de balance de los FIC.

Cabe mencionar que dentro de los indicadores con mayor coeficiente compuesto se encuentran variables de baja frecuencia como anual, bianual y trianual, por encima de indicadores con alta frecuencia y de las variables cuya tendencia de largo plazo fue extraída a partir del filtro de Hodrick-Prescott, lo cual va en línea con los resultados obtenidos por Lang et al. (2019).

\section{Indicador de alerta temprana para la detección de vulnerabilidades de los FIC - IATE FIC}

Tras la escogencia de los mejores indicadores por categoría, a partir del coeficiente compuesto, se procede al cálculo del indicador de alerta temprana para la detección de vulnerabilidades de los FIC.

Para la consecución de este objetivo es necesario estandarizar los indicadores seleccionados a partir de la sustracción de la media y la división por la desviación estándar de cada indicador ${ }^{14}$. El proceso anterior se realiza con el fin de partir de una escala homogénea en todas las variables que van a formar parte del indicador final y, así, facilitar su interpretación, la cual se hará en términos del número de desviaciones estándar que el indicador se aleja de un comportamiento normal de los FIC.

A su vez, cada indicador no posee la misma ponderación en el indicador de vulnerabilidad final, es necesario estimar la participación de cada uno mediante una regresión de mínimos cuadrados ordinarios (MCO) con tres restricciones,

13 En el anexo 3 se encuentran los resultados del coeficiente compuesto para todas las variables evaluadas.

14 En el trabajo realizado por Lang et al. (2019), la normalización se realizó a partir de la mediana de la distribución de cada indicador dado que los datos provenían de una muestra de países cuyo comportamiento no necesariamente es homogéneo. 
a saber: que la suma de los coeficientes de cada variable sea igual a 1, que los coeficientes sean mayores a 0 , que cada variable del indicador cuente con un peso mínimo de $5 \%$. Cabe mencionar que la variable dependiente para la estimación del modelo propuesto es la variable de vulnerabilidad calculada de acuerdo con lo señalado en la sección 2.1.

IATE FIC $=\beta 1($ Estructura de balance FIC $)+$ $\beta 2($ Desbalances Macroeconómicos $)+\beta 3($ Mercados Financieros $)+$ $\beta 4($ Liquidez FIC $)+\beta 5$ (Rentabilidad FIC $)+$ $\beta 6$ (Dinero Circulante en la Economía)

Donde $\beta i \geq 0,05, \sum_{i=1}^{6} \beta i=1$.

Al reemplazar los indicadores seleccionados a partir del coeficiente compuesto, la expresión (6) para la estimación por MCO sería la siguiente:

IATE FIC $=\beta 1($ Variación Anual Money Flow $)+$ $\beta 2($ Crecimiento Bianual ISE $)+\beta 3($ Crecimiento anual Brent $)+$ $\beta 4($ Crecimiento Trianual ALAC $)+$ $\beta 5$ (Cambio Anual Rentabilidad Semestral FIC) + $\beta 6($ Crecimiento Trianual M3)

Tras la estimación del modelo por MCO, todas las variables arrojan coeficientes estadísticamente significativos al 99,9\% de confianza, y se obtienen las siguientes ponderaciones para las seis variables que hacen parte del indicador de alertas tempranas de los FIC (IATE FIC).

IATE FIC $=0,054($ Variación Anual Money Flow $)+$ 0,171(Crecimiento Bianual ISE) + $0,195$ (Crecimiento anual Brent $)+$ 0,264(Crecimiento Trianual ALAC) +

0,153(Cambio Anual Rentabilidad Semestral FIC) + 0,163(Crecimiento Trianual M3)

En la figura 6 se muestra el indicador de alertas tempranas de los FIC (IATE FIC), aplicando los resultados presentados en la ecuación (8) a los datos históricos 
de las seis variables que hacen parte del modelo. Las áreas resaltadas corresponden a los periodos de vulnerabilidad identificados en la sección 2.1.

Figura 6: Indicador de Alertas Tempranas de los FIC (IATE FIC)

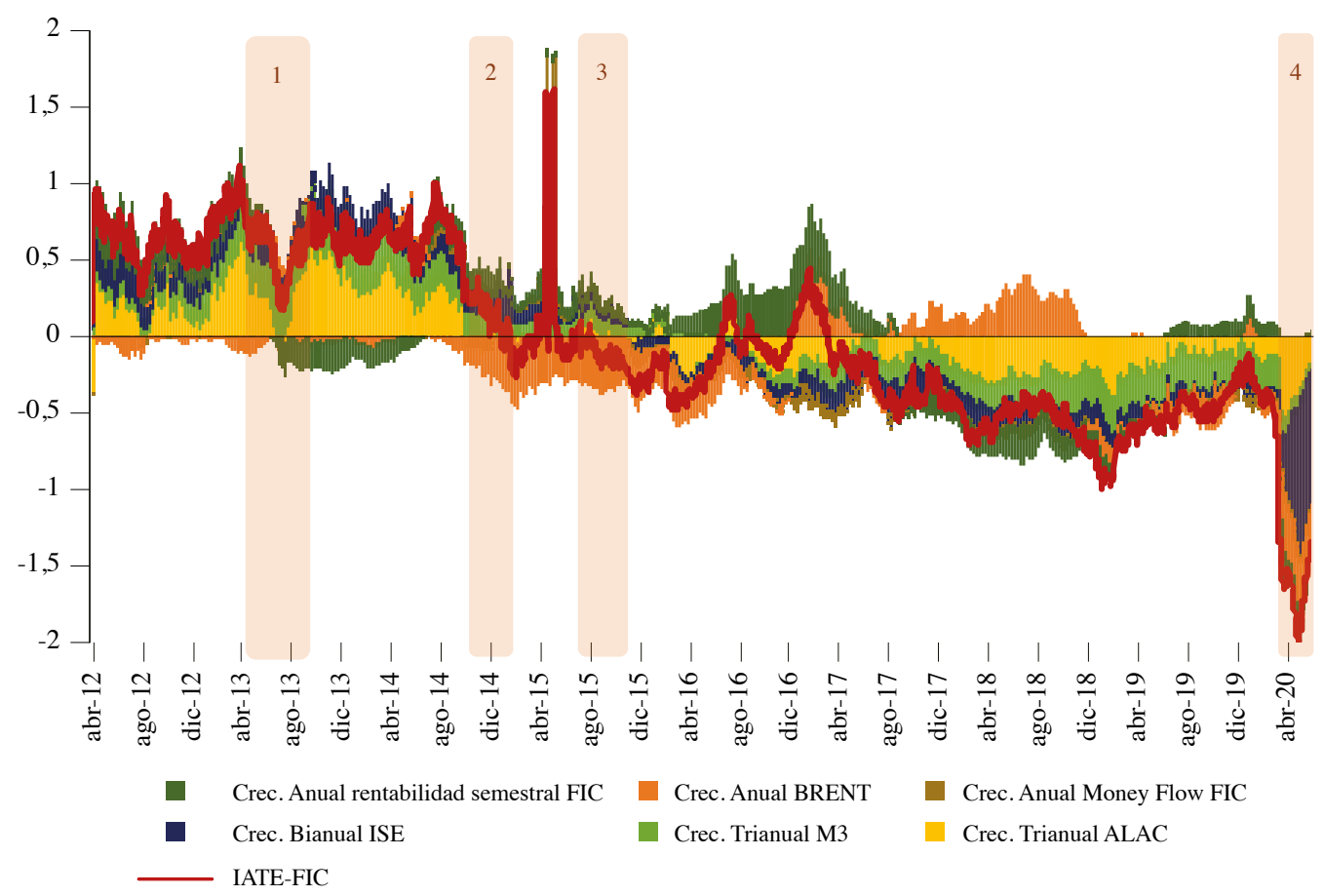

Fuente: cálculos propios.

Al analizar cada uno de los cuatro periodos de vulnerabilidad identificados, se observa que el indicador de alertas tempranas de los FIC (IATE FIC) presenta un incremento de entre 0,4 y 0,6 puntos aproximadamente, evidencia de una acumulación de vulnerabilidades (recalentamiento) que terminó por generar un escenario de fragilidad en los FIC. A su vez, el incremento mencionado se dio en promedio cuatro meses antes del inicio del periodo de fragilidad ${ }^{15}$, tiempo prudente para que las autoridades financieras tomen medidas que permitan mitigar el efecto de la vulnerabilidad en los FIC.

15 En el siguiente apartado del documento se analiza cuál es la ventana exacta de anticipación del IATE FIC, así como la magnitud de su incremento que permite predecir un periodo venidero de fragilidad en los FIC. 
Un ejemplo de las acciones que pueden tomar los hacedores de política se dio en el caso de la fragilidad generada en marzo de 2020 como consecuencia de los efectos del covid-19, donde el Banco de la República de Colombia le permitió a los FIC el acceso a las facilidades de liquidez de corto plazo, a partir de operaciones garantizadas con títulos de deuda privada.

A nivel general hay tres aspectos claves para la interpretación del indicador de alertas tempranas. El primero consiste en tendencias alcistas del indicador, las cuales dan señales de recalentamiento e incremento en las fuentes de vulnerabilidad (categorías de riesgo). El segundo corresponde a los cambios de tendencia alcista a bajista del IATE FIC, lo cual indica que posiblemente ocurra un evento de fragilidad en los FIC en los próximos meses. Finalmente, la composición del área indica la contribución a la vulnerabilidad de cada una de las categorías de riesgo seleccionadas, es decir, el aporte al recalentamiento.

De manera desagregada, en la figura 6 se evidencia que previo al primer periodo de fragilidad se presentó una acumulación de vulnerabilidad, principalmente en la categoría de Liquidez de los FIC, reflejado en la variable de crecimiento trianual de los ALAC. Lo anterior fue consecuencia de la alta volatilidad del mercado frente a los anuncios de contracción en la política monetaria expansiva de Estados Unidos y sus efectos de desvalorización en los activos líquidos de los FIC. Por su parte, antes del inicio del segundo periodo de vulnerabilidad se observó que el incremento del IATE FIC se da en adición al efecto de los ALAC, por el aporte de los componentes de dinero circulante en la economía (M3) y desbalances macroeconómicos (ISE).

Ahora, al analizar la acumulación de vulnerabilidades antes del tercer periodo de fragilidad se destaca un incremento significativo en el componente de estructura de balance de los FIC, reflejado en el indicador de money flow, el cual evidenció el nerviosismo de los inversionistas ante el fin del superciclo de los commodities, que los condujo a buscar inversiones en activos refugio, lo que afectó el comportamiento de los FIC.

Finalmente, previo al evento de fragilidad desatado por los efectos del covid-19, en los meses anteriores se observó un incremento del IATE FIC en respuesta principalmente a las categorías de rentabilidad de los FIC y mercados financieros (Brent). Cabe destacar que este evento de fragilidad fue consecuencia principalmente de un hecho exógeno al modelo, que no se encuentra recogido por ninguna de las categorías de riesgo planteadas, razón por la cual la señal emitida por el IATE FIC no presenta la misma contundencia de los eventos pasados. 
Cabe destacar que, si bien por la construcción del modelo existe la posibilidad de arrojar falsas alertas tempranas, los picos significativos del IATE FIC que no fueron asociados con periodos de fragilidad anticipan choques adversos en el comportamiento de los FIC y, por tanto, no deben ser catalogados como falsas alarmas. Por ejemplo, entre finales de 2016 y principios de 2017, el IATE FIC registró un alza de aproximadamente 0,6 puntos; posteriormente, entre junio y septiembre de 2017, los FIC pasaron de presentar un crecimiento trimestral del orden del 18 al $0 \%$.

\section{Ventana de anticipación y magnitud del impacto en los FIC}

Con el fin de medir con exactitud la ventana de tiempo con que el IATE FIC emite la alerta temprana y, a su vez, la magnitud del choque que predice en los FIC, este apartado expone la estimación de un modelo de vectores autorregresivos (VAR).

Cabe mencionar que en trabajo de Lang et al. (2019), el cual sirve como base para el presente documento, la estimación de la magnitud del impacto y el tiempo de anticipación se realizó a partir del modelo de proyecciones locales de Jordà (2005). Sin embargo, al comparar los resultados de las dos metodologías se encontró que arrojan resultados similares, por tanto, para este trabajo se presenta el modelo VAR (modelo vectorial autorregresivo).

A grandes rasgos, un modelo VAR estima un grupo de ecuaciones simultáneas compuesto por diferentes variables que cumplen el rol tanto de variable dependiente como independiente en el sistema de ecuaciones. Por lo general, las variables explicativas de las diferentes ecuaciones simultáneas son las mismas y corresponden a los rezagos de las distintas variables que se utilizan en el modelo. El resultado del sistema de ecuaciones permite identificar las diferentes relaciones de causalidad existentes entre las variables. A partir del trabajo de Sims (1980) se infiere que las principales ventajas de los modelos VAR se enfocan en el pronóstico de series de tiempo, su aplicación en modelos económicos y en la evaluación de políticas económicas.

Una ventaja adicional que ofrecen los modelos VAR es la posibilidad de la estimación de las funciones impulso-respuesta, las cuales expresan, a partir de las interacciones de las variables obtenidas en los modelos VAR, la reacción simultánea de las variables ante un choque en las mismas. Es decir, la respuesta de las variables ante un impulso dado. 
Los resultados de las funciones-impulso respuesta obtenidas a partir de la aplicación de los modelos VAR reflejan la variación que se observará en un periodo de tiempo determinado en el VNF, a partir de la ocurrencia de un choque en el IATE FIC (figura 7).

Figura 7: Impulso-respuesta del VNF ante choque en IATE FIC

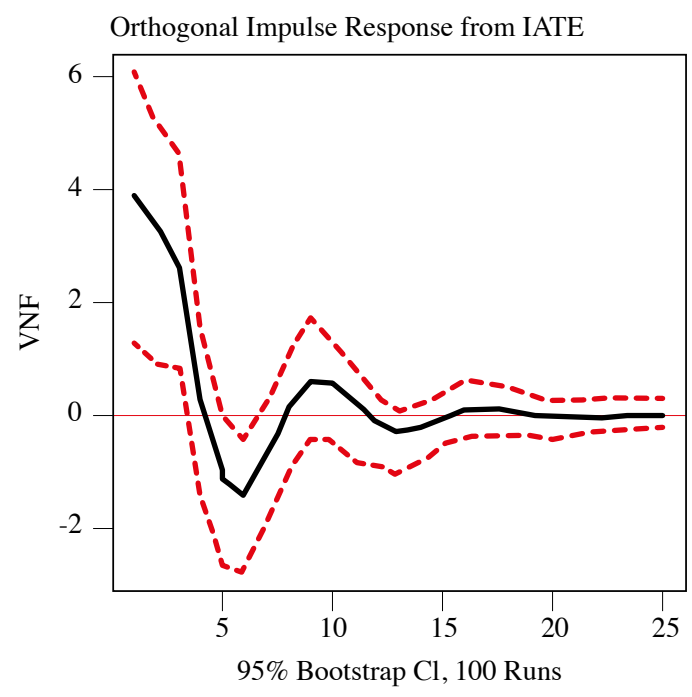

Fuente: cálculos propios.

La figura 7 expone la reacción de la variación trimestral del VNF ante el choque de una desviación estándar en el IATE FIC. Se espera que el mayor impacto en el VNF ocurra seis meses después de un choque en el IATE FIC. La disminución esperada en el crecimiento trimestral del VNF corresponde a 1,4 puntos porcentuales por cada incremento de 0,2 puntos en el IATE FIC.

Con el resultado mencionado se cumple el objetivo de alerta temprana en la cual se calcula la ventana exacta de anticipación del IATE FIC y la magnitud del impacto esperado en el comportamiento de los FIC, elementos de gran ayuda para los hacedores de política en la toma de decisiones ante escenarios de incertidumbre en el comportamiento futuro de la industria de FIC.

\section{Conclusiones}

Con la evolución de los mercados financieros, los fondos de inversión se han convertido en un agente importante del mercado, alcanzando el nivel de agentes 
sistémicos en algunos mercados o países. Dada esta situación, es necesario contar con herramientas que permitan una adecuada y oportuna identificación de los riesgos a los cuales se encuentran expuestos dichos fondos.

Sin embargo, al observar la literatura de sistemas de alertas tempranas se encuentra que el principal enfoque está dirigido hacia la industria bancaria, lo que deja de lado los efectos en la estabilidad financiera que pueden traer consigo industrias como la de los FIC.

Teniendo en cuenta el grado de aprobación en la literatura de los sistemas de alerta temprana para el sector bancario, el presente trabajo planteó como objetivo general implementar la metodología propuesta por Lang et al. (2019) con el fin de desarrollar un indicador de alerta temprana que anticipe los escenarios de vulnerabilidad en los FIC en Colombia.

Para cumplir de manera adecuada con el objetivo planteado, dentro de los objetivos específicos se consideró que la metodología propuesta permitiera predecir con una ventana de tiempo considerable el momento y la severidad de una posible vulnerabilidad en los FIC y la descomposición de las fuentes de riesgo que ocasionan la fragilidad.

A partir de los resultados obtenidos se infiere que el indicador de alerta temprana (IATE FIC) cumple con su objetivo de detección de vulnerabilidades en los FIC con un periodo de tiempo considerable para la toma de decisiones, lo que permite la medición de la ventana exacta de anticipación, la magnitud del impacto esperado y la principal fuente de riesgo del evento de estrés. Ante un choque de una desviación estándar en el IATE FIC, aproximadamente un incremento de 0,2 puntos del indicador, se espera una caída de 1,4 puntos porcentuales en el crecimiento trimestral del valor neto de los FIC (VNF) en los seis meses siguientes.

Para la construcción del indicador de alertas tempranas se tuvieron en cuenta cinco etapas: identificación de periodos de vulnerabilidad a partir del vNF, selección de categorías de riesgo (estructura de balance, liquidez y rentabilidad de los FIC; dinero circulante en la economía; mercados financieros y desbalances macroeconómicos), selección de indicadores por categoría con base en el poder univariado de predicción (AUROC y utilidad relativa), cálculo de las ponderaciones de cada categoría y cálculo del indicador de alerta temprana (IATE FIC).

Los hallazgos encontrados en el presente trabajo representan un avance importante en la detección de vulnerabilidades de los fondos de inversión y pueden ser de gran ayuda para los hacedores de política en la toma de decisiones ante escenarios de incertidumbre en el comportamiento futuro de la industria de FIC. 


\section{Referencias}

Alessi, L. y Detken, C. (2011). Quasi real time early warning indicators for costly asset price boom/bust cycles: A role for global liquidity. European Journal of Political Economy, 27(3), 520-533. https://doi.org/10.1016/j.ejpoleco.2011.01.003

Backus, D. K. y Kehoe, P. J. (1992). International evidence on the historical properties of business cycles. The American Economic Review, 82(4), 864-888. https://www. jstor.org/stable/2117348

Bussière, M. y Fratzscher, M. (2008). Low probability, high impact: Policy making and extreme events. Journal of Policy Modeling, 30(1), 111-121. https://doi.org/10.1016/j. jpolmod.2007.03.007

Consultative Document (2nd) (2015). Assessment Methodologies for Identifying NonBank Non-Insurer Global Systemically Important Financial Institutions Proposed High-Level Framework and Specific Methodologies.

Das, N., Kish, R. y Muething, D. (2005). Modeling Hedge Fund Returns. Financial Decisions, Article 4.

Demirgüç-Kunt, A. y Detragiache, E. (2000). Monitoring banking sector fragility: A multivariate logit approach. World Bank Economic Review, 14(2), 287-307. https:// doi.org/10.1093/wber/14.2.287

Division of Investment Management Risk and Examinations Office Risk and Examinations Office (2017). Private Funds Statistics.

Federal Deposit Insurance Corporation (2016). 12 CFR Part 327 Assessments; Final Rule mstockstill on DSK3G9T082PROD with RULES3.

FIAFIN (n.d.). Estadísticas por País-Tipo. http://www.fiafin.org/estadistica/showPaisTipo

Fuertes, A. M. y Kalotychou, E. (2007). Optimal design of early warning systems for sovereign debt crises. International Journal of Forecasting, 23(1), 85-100. https:// doi.org/10.1016/j.ijforecast.2006.07.001

Galindo Vergara, M., Serna, O., Garcés, L., Vicente González, J., Muñoz, J. y Peláez, N. (n.d.). Reportes Fogafin Sistema de primas ajustadas por riesgo. Fogafin. 
Hentati, R., Kaffel, A. y Prigent, J.-L. (2010). Dynamic versus static optimization of hedge fund portfolios: The relevance of performance measures. International Journal of Business, 15(1), 1-17. http://www.craig.csufresno.edu/IJB/Recent Pub.htm

Investment Management Quality Ratings (n.d.). https://www.fitchratings.com/research/ es/fund-asset-managers/investment-management-quality-ratings-27-05-2020

Jin, X. y Simone, F. N. (2015). Investment funds? vulnerabilities: A tail-risk dynamic CIMDO approach. In BCL working papers. https://ideas.repec.org/p/bcl/bclwop/ bclwp095.html

Jordà, Ò. (2005). Estimation and inference of impulse responses by local projections. American Economic Review, 95(1), 161-182. https://doi.org/10.1257/0002828053828518

Kaiser, D. G., Schweizer, D. y Wu, L. (2012). Efficient hedge fund strategy allocations-Systematic framework for investors that incorporates higher moments. In Financial Markets, Institutions \& Instruments, 21(5), 241-260. http://onlinelibrary. wiley.com/journal/10.1111/(ISSN)1468-0416/issues

Koellner, T., Weber, O., Fenchel, M. y Scholz, R. (2005). Principles for sustainability rating of investment funds. Business Strategy and the Environment, 14(1), 54-70. https://doi.org/10.1002/bse.423

Lang, J. H., Izzo, C., Fahr, S. y Ruzicka, J. (2019). Occasional Paper Series Anticipating the bust: A new cyclical systemic risk indicator to assess the likelihood and severity of financial crises. Office of the European Union. https://doi.org/10.2866/468656

Leyva Rayón, E. (2015). Modelo multifactor para analizar la exposición de los hedge funds a factores de riesgo macroeconómico. Economía Teoría y Práctica, 42, 9-44. https://doi.org/10.24275/etypuam/ne/422015/leyva

Markowitz, H. M. (1959). Portfolio selection: Efficient diversification of investments. Yale University Press. http://www.jstor.org/stable/j.ctt1bh4c8hj

Ramírez, S., Jiménez, J. S. y Witmer, J. (2015). Canadian Open-End Mutual Funds: An Assessment of Potential Vulnerabilities. Bank of Canada System Review, 47-56.

Sarlin, P. (2013). On policymakers' loss functions and the evaluation of early warning systems. Economics Letters, 119(1), 1-7. https://doi.org/10.1016/j.econlet.2012.12.030 
Sims, C. A. (1980). Macroeconomics and reality. Econometrica, 48(1), 1-48. https:// doi.org/10.2307/1912017

Swets, J. A. (1996). Signal detection theory and ROC analysis in psychology and diagnostics: Collected papers. Lawrence Erlbaum Associates. https://books.google. com.co/books?id=1vkmaqaamaaj

\section{Anexos}

\section{Anexo 1. Tratamiento de datos}

Debido a que la periodicidad de los datos de los FIC es diaria, es necesario ampliar la frecuencia de varios indicadores evaluados. A continuación, se presenta la lista de variables, sus fuentes y el tratamiento realizado.

\begin{tabular}{|c|c|c|c|c|c|}
\hline Variable & Sigla & Fuente & $\begin{array}{l}\text { Inicio de } \\
\text { la serie }\end{array}$ & $\begin{array}{c}\text { Periodicidad } \\
\text { original }\end{array}$ & $\begin{array}{l}\text { Transformación } \\
\text { realizada }\end{array}$ \\
\hline $\begin{array}{l}\text { Tasa de cambio } \\
\text { representativa del } \\
\text { mercado }\end{array}$ & TRM & $\begin{array}{l}\text { Banco de la } \\
\text { República de } \\
\text { Colombia }\end{array}$ & $1 / 01 / 2008$ & Diaria & $\mathrm{NA}$ \\
\hline $\begin{array}{l}\text { Índice Accionario } \\
\text { de Capitalización } \\
\text { COLCAP }\end{array}$ & COLCAP & $\begin{array}{l}\text { Banco de la } \\
\text { República de } \\
\text { Colombia }\end{array}$ & $15 / 01 / 2008$ & Diaria & $\mathrm{NA}$ \\
\hline Petróleo BRENT & BRENT & Bloomberg & $22 / 07 / 2008$ & Diaria & NA \\
\hline $\begin{array}{l}\text { Tasa cero cupón en } \\
\text { pesos TES a } 1 \text { año }\end{array}$ & TES $\_1_{-}$AÑO & $\begin{array}{l}\text { Banco de la } \\
\text { República de } \\
\text { Colombia }\end{array}$ & $1 / 01 / 2008$ & Diaria & NA \\
\hline $\begin{array}{l}\text { Tasa cero cupón en } \\
\text { pesos TES a } 5 \text { año }\end{array}$ & $\begin{array}{l}\text { TES }_{-}{ }^{5}- \\
\text { AÑOS }\end{array}$ & $\begin{array}{l}\text { Banco de la } \\
\text { República de } \\
\text { Colombia }\end{array}$ & $1 / 01 / 2008$ & Diaria & NA \\
\hline $\begin{array}{l}\text { Tasa cero cupón en } \\
\text { pesos TES a } 10 \text { año }\end{array}$ & $\begin{array}{l}\text { TES_ } 10 \\
\text { AÑOS }\end{array}$ & $\begin{array}{l}\text { Banco de la } \\
\text { República de } \\
\text { Colombia }\end{array}$ & $1 / 01 / 2008$ & Diaria & NA \\
\hline $\begin{array}{l}\text { Tasas de captación } \\
\text { CDT a } 30 \text { días }\end{array}$ & $\begin{array}{l}\mathrm{CDT}_{-} 30_{-} \\
\text {DÍAS }\end{array}$ & $\begin{array}{l}\text { Banco de la } \\
\text { República de } \\
\text { Colombia }\end{array}$ & $1 / 04 / 2008$ & Diaria & NA \\
\hline
\end{tabular}




\begin{tabular}{|c|c|c|c|c|c|}
\hline Variable & Sigla & Fuente & $\begin{array}{l}\text { Inicio de } \\
\text { la serie }\end{array}$ & $\begin{array}{c}\text { Periodicidad } \\
\text { original }\end{array}$ & $\begin{array}{l}\text { Transformación } \\
\text { realizada }\end{array}$ \\
\hline $\begin{array}{l}\text { Tasas de captación } \\
\text { CDT a } 90 \text { días }\end{array}$ & $\begin{array}{l}\text { CDT }-90 ~ \\
\text { DÍAS }\end{array}$ & $\begin{array}{l}\text { Banco de la } \\
\text { República de } \\
\text { Colombia }\end{array}$ & $1 / 04 / 2008$ & Diaria & NA \\
\hline $\begin{array}{l}\text { Tasas de captación } \\
\text { CDT a } 180 \text { días }\end{array}$ & $\begin{array}{l}\text { CDT_- } 180{ }_{-} \\
\text {DÍAS }\end{array}$ & $\begin{array}{l}\text { Banco de la } \\
\text { República de } \\
\text { Colombia }\end{array}$ & $1 / 04 / 2008$ & Diaria & NA \\
\hline $\begin{array}{l}\text { Tasas de captación } \\
\text { CDT mayor o igual a } \\
360 \text { días }\end{array}$ & $\begin{array}{l}\mathrm{CDT}_{-}+360_{-} \\
\text {DÍAS }\end{array}$ & $\begin{array}{l}\text { Banco de la } \\
\text { República de } \\
\text { Colombia }\end{array}$ & $1 / 04 / 2008$ & Diaria & NA \\
\hline $\begin{array}{l}\text { Depósitos de los } \\
\text { Establecimientos de } \\
\text { Crédito }\end{array}$ & Depósitos & $\begin{array}{l}\text { Banco de la } \\
\text { República de } \\
\text { Colombia }\end{array}$ & $1 / 01 / 2008$ & Semanal & Interpolación Lineal \\
\hline $\begin{array}{l}\text { Agregado mone- } \\
\text { tario M3: Efectivo } \\
+ \text { pasivos sujetos a } \\
\text { encaje (PSE) }\end{array}$ & M3 & $\begin{array}{l}\text { Banco de la } \\
\text { República de } \\
\text { Colombia }\end{array}$ & $1 / 01 / 2008$ & Semanal & Interpolación Lineal \\
\hline $\begin{array}{l}\text { Base Monetaria: } \\
\text { Efectivo + Reserva } \\
\text { Bancaria (Efec- } \\
\text { tivo en caja del } \\
\text { sistema financiero } \\
\text { + depósitos del } \\
\text { sistema financiero } \\
\text { en el Banco de la } \\
\text { República) }\end{array}$ & $\begin{array}{l}\text { Base_mone- } \\
\text { taria }\end{array}$ & $\begin{array}{l}\text { Banco de la } \\
\text { República de } \\
\text { Colombia }\end{array}$ & $1 / 01 / 2008$ & Semanal & Interpolación Lineal \\
\hline Tasa de desempleo & Desempleo & $\begin{array}{l}\text { Banco de la } \\
\text { República de } \\
\text { Colombia }\end{array}$ & $1 / 01 / 2008$ & Mensual & Interpolación Lineal \\
\hline $\begin{array}{l}\text { Índice de segui- } \\
\text { miento a la econo- } \\
\text { mía }\end{array}$ & ISE & DANE & $1 / 01 / 2008$ & Mensual & Interpolación Lineal \\
\hline Efectivo FIC & EFECTIVO & $\begin{array}{l}\text { Superintenden- } \\
\text { cia Financiera } \\
\text { de Colombia }\end{array}$ & $1 / 04 / 2009$ & Diaria & NA \\
\hline $\begin{array}{l}\text { Activos líquidos de } \\
\text { alta calidad FIC }\end{array}$ & ALAC & $\begin{array}{l}\text { Superintenden- } \\
\text { cia Financiera } \\
\text { de Colombia }\end{array}$ & $1 / 04 / 2009$ & Diaria & $\begin{array}{l}\text { Se aproxima como } \\
\text { la suma del efectivo } \\
\text { más el } 7 \% \text { de las } \\
\text { inversiones }\end{array}$ \\
\hline
\end{tabular}




\begin{tabular}{|c|c|c|c|c|c|}
\hline Variable & Sigla & Fuente & $\begin{array}{l}\text { Inicio de } \\
\text { la serie }\end{array}$ & $\begin{array}{c}\text { Periodicidad } \\
\text { original }\end{array}$ & $\begin{array}{l}\text { Transformación } \\
\text { realizada }\end{array}$ \\
\hline $\begin{array}{l}\text { Máximo requeri- } \\
\text { miento de liquidez } \\
\text { FIC }\end{array}$ & MRLQ & $\begin{array}{l}\text { Superintenden- } \\
\text { cia Financiera } \\
\text { de Colombia }\end{array}$ & $1 / 04 / 2009$ & Diaria & $\begin{array}{l}\text { Se aproxima como } \\
\text { el } 10 \% \text { del VNF, tal } \\
\text { como se establece en } \\
\text { el Anexo } 3 \text { del Capí- } \\
\text { tulo VI de la Circular } \\
\text { Básica Contable y } \\
\text { Financiera }\end{array}$ \\
\hline $\begin{array}{l}\text { Valor neto del fondo } \\
\text { FIC }\end{array}$ & VNF & $\begin{array}{l}\text { Superintenden- } \\
\text { cia Financiera } \\
\text { de Colombia }\end{array}$ & $1 / 04 / 2009$ & Diaria & $\mathrm{NA}$ \\
\hline Inversiones FIC & INVERSIONES & $\begin{array}{l}\text { Superintenden- } \\
\text { cia Financiera } \\
\text { de Colombia }\end{array}$ & $1 / 04 / 2009$ & Diaria & NA \\
\hline $\begin{array}{l}\text { Money Flow: Acu- } \\
\text { mulado } 12 \text { meses de } \\
\text { los aportes y retiros } \\
\text { netos }\end{array}$ & $\begin{array}{l}\text { APORT_- } \\
\text { RET_anual }\end{array}$ & $\begin{array}{l}\text { Superintenden- } \\
\text { cia Financiera } \\
\text { de Colombia }\end{array}$ & $1 / 04 / 2009$ & Diaria & $\begin{array}{l}\text { NA. Dado que se pue- } \\
\text { den presentar acu- } \\
\text { mulados negativos, } \\
\text { para el cálculo de las } \\
\text { variaciones se utili- } \\
\text { zó como denomina- } \\
\text { dor el valor absoluto } \\
\text { del money flow } \\
\text { para la periodicidad } \\
\text { correspondiente. Por } \\
\text { ejemplo, la variación } \\
\text { anual del money } \\
\text { flow se calculó de } \\
\text { la siguiente manera } \\
\{[(\text { money flowt- } \\
\text { money flowt-365)/ } \\
\text { abs(money } \\
\text { flowt-365)] }\}\end{array}$ \\
\hline $\begin{array}{l}\text { Rentabilidad men- } \\
\text { sual FIC }\end{array}$ & $\begin{array}{l}\text { Rent_men- } \\
\text { sual }\end{array}$ & $\begin{array}{l}\text { Superintenden- } \\
\text { cia Financiera } \\
\text { de Colombia }\end{array}$ & $1 / 01 / 2008$ & Diaria & NA \\
\hline $\begin{array}{l}\text { Rentabilidad semes- } \\
\text { tral FIC }\end{array}$ & $\begin{array}{l}\text { Rent_semes- } \\
\text { tral }\end{array}$ & $\begin{array}{l}\text { Superintenden- } \\
\text { cia Financiera } \\
\text { de Colombia }\end{array}$ & $1 / 01 / 2008$ & Diaria & $\mathrm{NA}$ \\
\hline $\begin{array}{l}\text { Rentabilidad anual } \\
\text { FIC }\end{array}$ & Rent_anual & $\begin{array}{l}\text { Superintenden- } \\
\text { cia Financiera } \\
\text { de Colombia }\end{array}$ & $1 / 01 / 2008$ & Diaria & NA \\
\hline
\end{tabular}




\begin{tabular}{|c|c|c|c|c|c|}
\hline Variable & Sigla & Fuente & $\begin{array}{l}\text { Inicio de } \\
\text { la serie }\end{array}$ & $\begin{array}{c}\text { Periodicidad } \\
\text { original }\end{array}$ & $\begin{array}{l}\text { Transformación } \\
\text { realizada }\end{array}$ \\
\hline $\begin{array}{l}\text { Rendimientos } \\
\text { abonados: acumula- } \\
\text { do } 12 \text { meses de las } \\
\text { utilidades de los FIC }\end{array}$ & $\begin{array}{l}\text { Rendim_ } \\
\text { abon_anual }\end{array}$ & $\begin{array}{l}\text { Superintenden- } \\
\text { cia Financiera } \\
\text { de Colombia }\end{array}$ & $1 / 04 / 2010$ & Diaria & $\begin{array}{l}\text { NA. Dado que se } \\
\text { pueden presentar } \\
\text { acumulados negati- } \\
\text { vos, para el cálculo } \\
\text { de las variaciones } \\
\text { se utilizó como de- } \\
\text { nominador el valor } \\
\text { absoluto de los ren- } \\
\text { dimientos abonados } \\
\text { para la periodicidad } \\
\text { correspondiente. Por } \\
\text { ejemplo, la variación } \\
\text { anual de los rendi- } \\
\text { mientos abonados } \\
\text { se calculó de la } \\
\text { siguiente manera } \\
\{[(\text { rendimientos abo- } \\
\text { nadost-rendimientos } \\
\text { abonadost-365)/ } \\
\text { abs(rendimientos } \\
\text { abonadost-365)]\} }\end{array}$ \\
\hline
\end{tabular}

\section{Anexo 2. AUROC de los indicadores seleccionados}
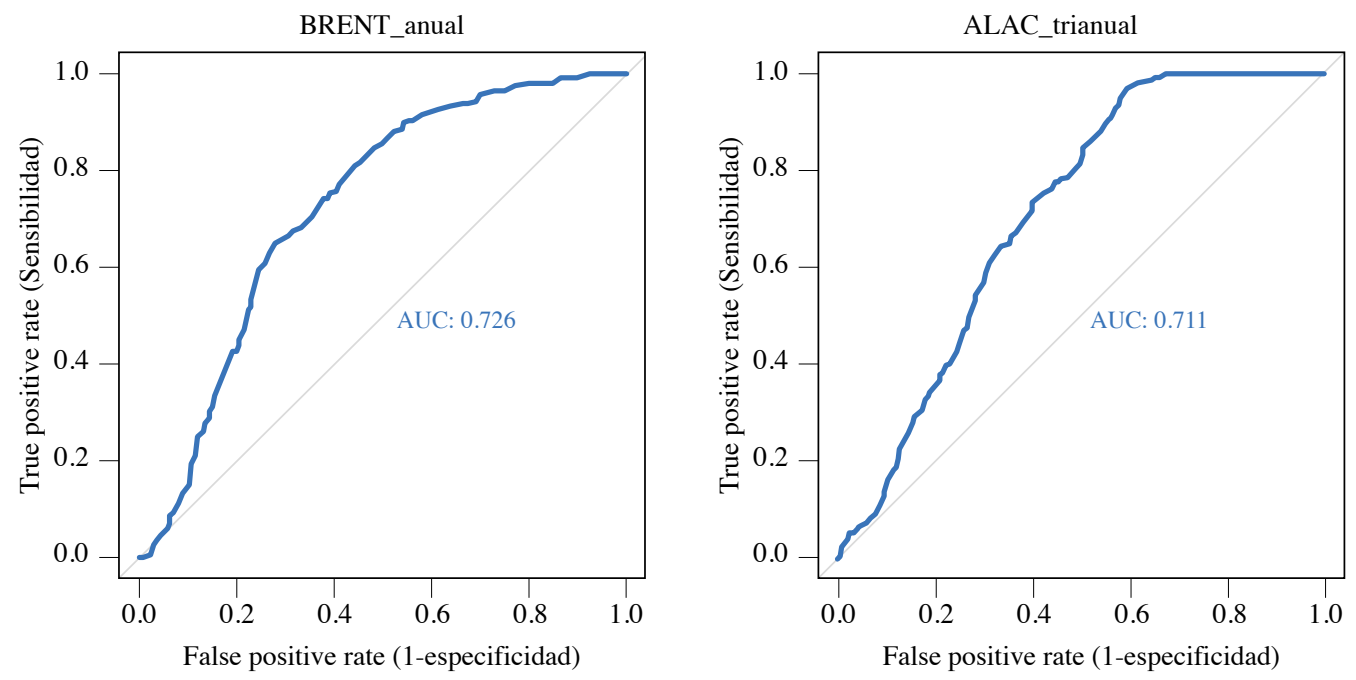

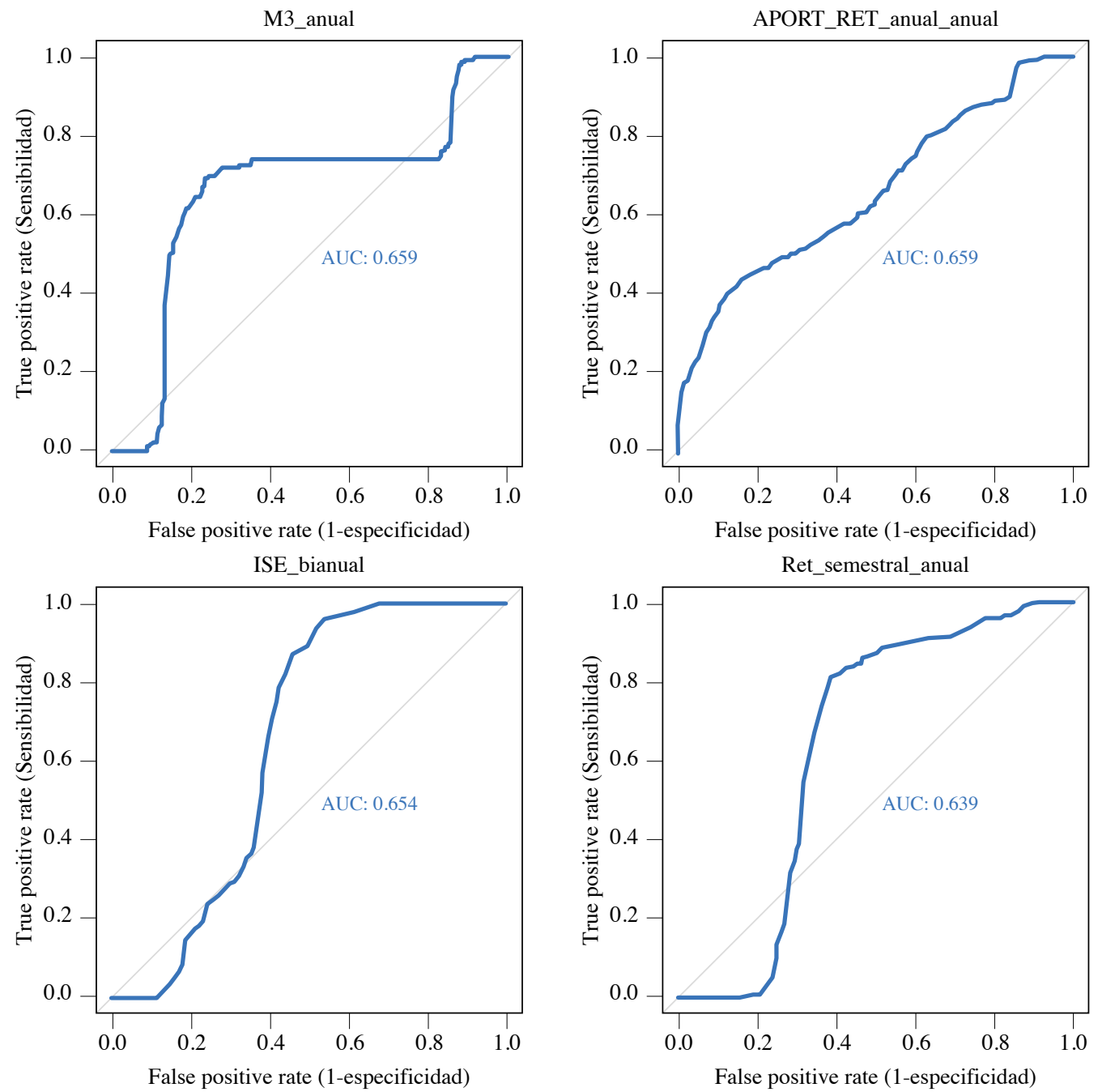

Fuente: cálculos propios. 


\begin{tabular}{|c|c|c|c|c|c|c|c|c|c|c|c|c|c|c|c|c|c|c|}
\hline 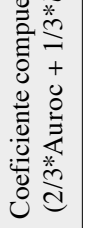 & 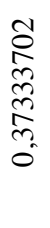 & 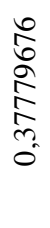 & 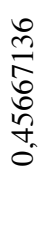 & 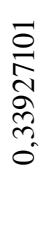 & 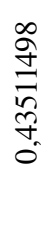 & \begin{tabular}{l}
$\infty$ \\
$\infty$ \\
$\infty$ \\
$\hat{n}$ \\
\multirow{0}{*}{} \\
0 \\
0
\end{tabular} & 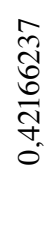 & 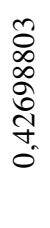 & 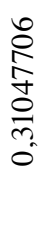 & 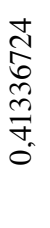 & \begin{tabular}{l} 
年 \\
$\tilde{n}$ \\
\multirow{J}{*}{} \\
0 \\
$m$ \\
0
\end{tabular} & 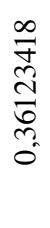 & 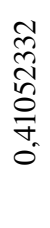 & 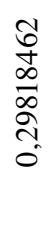 & $\begin{array}{l}\frac{m}{\pi} \\
\frac{J}{\sigma} \\
\frac{m}{2} \\
0\end{array}$ & 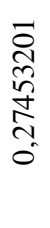 & 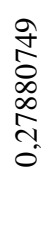 & 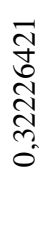 \\
\hline 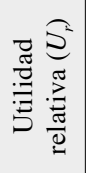 & 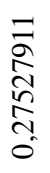 & 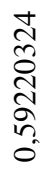 & 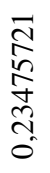 & 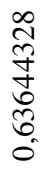 & 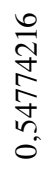 & 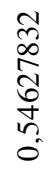 & $\begin{array}{l}\hat{2} \\
\hat{n} \\
\infty \\
\infty \\
n \\
n \\
0\end{array}$ & 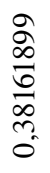 & 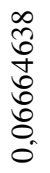 & $\begin{array}{l}\stackrel{+}{0} \\
\stackrel{0}{0} \\
\infty \\
\infty \\
\infty \\
\sim \\
\tilde{0}\end{array}$ & $\begin{array}{l}\frac{1}{5} \\
\frac{2}{n} \\
2 \\
n \\
0\end{array}$ & 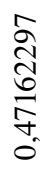 & 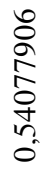 & 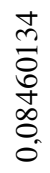 & $\begin{array}{l}n \\
\infty \\
\tilde{n} \\
\delta \\
\infty \\
0 \\
6 \\
0\end{array}$ & 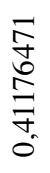 & 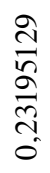 & 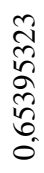 \\
\hline 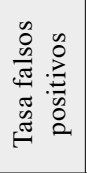 & 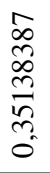 & $\tilde{O}_{0}$ & 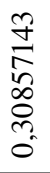 & 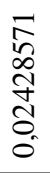 & 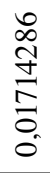 & 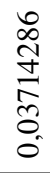 & $\begin{array}{l}J_{0} \\
\text {. }\end{array}$ & 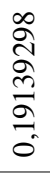 & 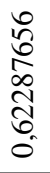 & 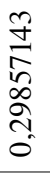 & 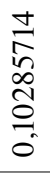 & \begin{tabular}{l}
0 \\
$\infty$ \\
\multirow{2}{*}{} \\
$\frac{\hat{\sigma}}{6}$ \\
0 \\
0
\end{tabular} & $\begin{array}{l}\text { రీ } \\
\hat{0} \\
\infty \\
0 \\
0 \\
0\end{array}$ & 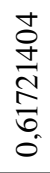 & 0 & 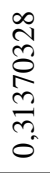 & 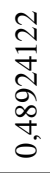 & 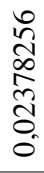 \\
\hline 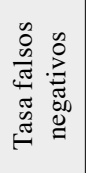 & 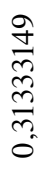 & 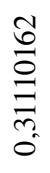 & 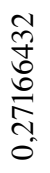 & 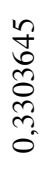 & 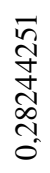 & 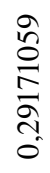 & 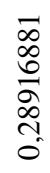 & 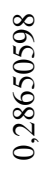 & 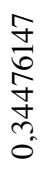 & 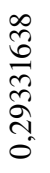 & 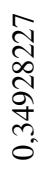 & 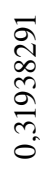 & 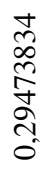 & 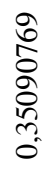 & 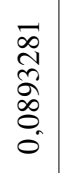 & 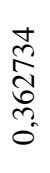 & 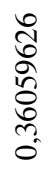 & $\begin{array}{l}2 \\
\infty \\
0 \\
\infty \\
\infty \\
\infty \\
0 \\
0\end{array}$ \\
\hline 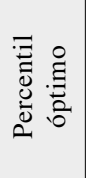 & 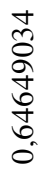 & $\begin{array}{l}\hat{a} \\
\hat{\sigma} \\
\text { ò } \\
\text { a. } \\
\hat{0}\end{array}$ & 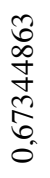 & 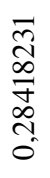 & 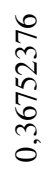 & 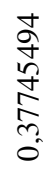 & 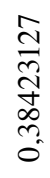 & $\begin{array}{l}\vec{a} \\
\frac{n}{n} \\
\stackrel{n}{n} \\
0\end{array}$ & $\begin{array}{l}1 \\
0 \\
0 \\
0 \\
0 \\
0 \\
\infty \\
0\end{array}$ & 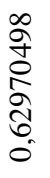 & 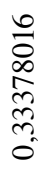 & 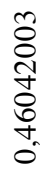 & 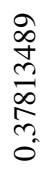 & \begin{tabular}{l}
+ \\
$\infty$ \\
\multirow{2}{人}{} \\
$\hat{2}$ \\
$\hat{n}$ \\
$\infty$ \\
0 \\
0
\end{tabular} & 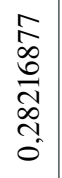 & $\begin{array}{l}\frac{n}{\sigma} \\
\frac{7}{7} \\
n \\
n \\
0\end{array}$ & 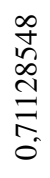 & 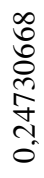 \\
\hline ○. & 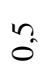 & $n$ & $\tilde{0}$ & $\tilde{n}$ & $\ddot{n}$ & $\tilde{0}$ & $n$ & $n$ & $n$ & $\ddot{n}$ & $\tilde{0}$ & $\ddot{n}$ & $n$ & $\tilde{0}$ & $\begin{array}{l}0 \\
\infty \\
0\end{array}$ & $\tilde{n}$ & $\tilde{n}$ & $\ddot{n}$ \\
\hline $\begin{array}{l}\text { o } \\
\text { ș } \\
\dot{4}\end{array}$ & 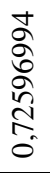 & 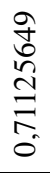 & 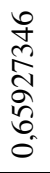 & $\begin{array}{l}\stackrel{m}{m} \\
\stackrel{2}{\circ} \\
\hat{\sigma} \\
\hat{\sigma} \\
0\end{array}$ & 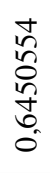 & 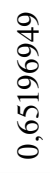 & $\begin{array}{l}\vec{\delta} \\
\infty \\
\infty \\
+ \\
\dot{0} \\
0\end{array}$ & 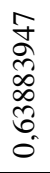 & 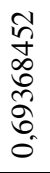 & 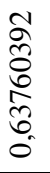 & 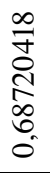 & 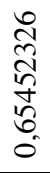 & 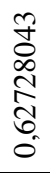 & 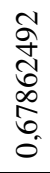 & 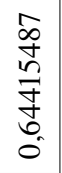 & 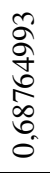 & 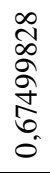 & \begin{tabular}{l} 
\\
$\hat{\sigma}$ \\
$\infty$ \\
$\infty$ \\
\multirow{0}{0}{} \\
0
\end{tabular} \\
\hline 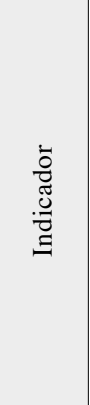 & 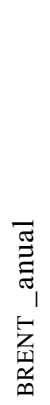 & 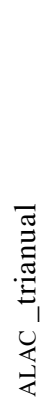 & 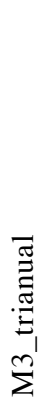 & 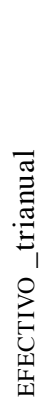 & 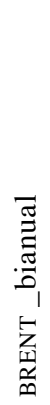 & 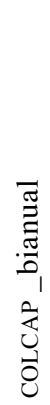 & 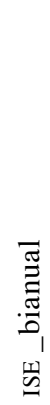 & 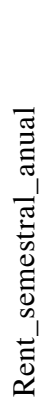 & 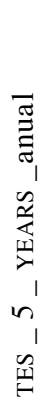 & 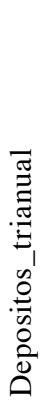 & 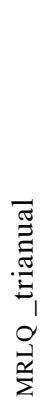 & 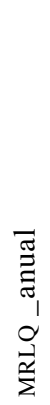 & 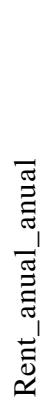 & 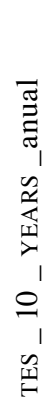 & 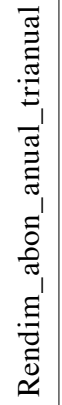 & 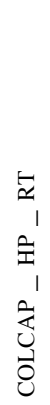 & 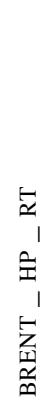 & 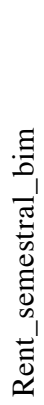 \\
\hline 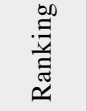 & - & $\sim$ & $m$ & $\nabla$ & $n$ & 0 & $r$ & $\infty$ & $a$ & 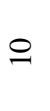 & $=$ & 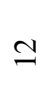 & $\cong$ & $\Xi$ & 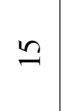 & 0 & I & $\stackrel{\infty}{\sim}$ \\
\hline
\end{tabular}




\begin{tabular}{|c|c|c|c|c|c|c|c|c|c|c|c|c|c|c|c|c|c|c|c|}
\hline 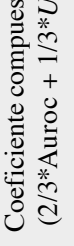 & 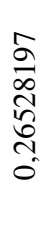 & 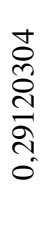 & 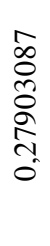 & 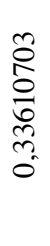 & 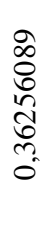 & $\begin{array}{l}\frac{J}{ \pm} \\
\stackrel{2}{\infty} \\
\infty \\
\infty \\
\infty \\
\text {. }\end{array}$ & 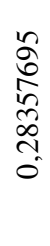 & 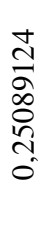 & 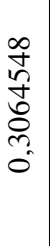 & $\begin{array}{l}\text { } \\
\infty \\
\infty \\
\infty \\
+ \\
\infty \\
\\
\tilde{c}\end{array}$ & 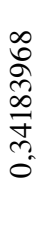 & $\begin{array}{l}\frac{n}{0} \\
\stackrel{0}{n} \\
\approx \\
\\
0\end{array}$ & 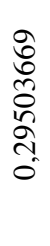 & 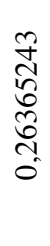 & 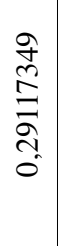 & 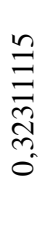 & 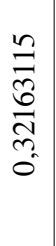 & 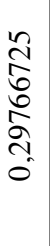 & 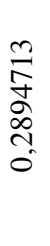 \\
\hline 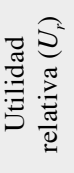 & 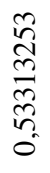 & 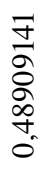 & $\begin{array}{l}\frac{m}{\sigma} \\
\text { bे } \\
\circ \\
0 \\
0 \\
0\end{array}$ & 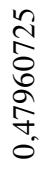 & 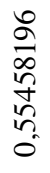 & $\begin{array}{l}\text { Ñ } \\
\tilde{n} \\
\infty \\
\infty \\
\sim \\
0 \\
0\end{array}$ & 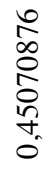 & 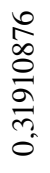 & 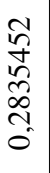 & 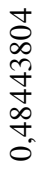 & 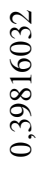 & 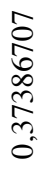 & 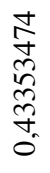 & 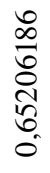 & 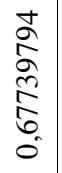 & 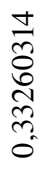 & 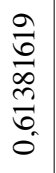 & 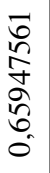 & 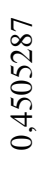 \\
\hline 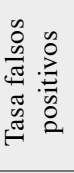 & 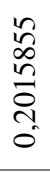 & 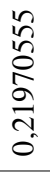 & $\begin{array}{l}\stackrel{0}{n} \\
0 \\
0\end{array}$ & 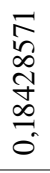 & $\begin{array}{l}\vec{\nabla} \\
i \\
\infty \\
\infty \\
\infty \\
0 \\
0 \\
0\end{array}$ & 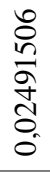 & 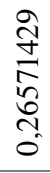 & $\stackrel{\text { fo }}{\circ}$ & $F_{0}$ & 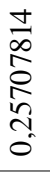 & $\begin{array}{l}0 \\
\text { N } \\
0\end{array}$ & 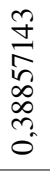 & 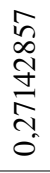 & \begin{tabular}{l}
$\overrightarrow{1}$ \\
D \\
$\infty$ \\
\multirow{1}{*}{} \\
$\infty$ \\
0 \\
0 \\
0
\end{tabular} & 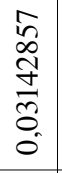 & 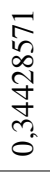 & 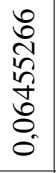 & 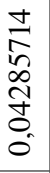 & 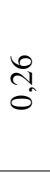 \\
\hline 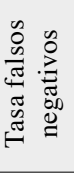 & 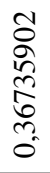 & 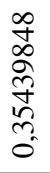 & 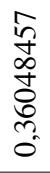 & 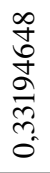 & $\frac{\sqrt[n]{n}}{\frac{\infty}{2}}$ & 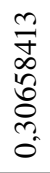 & 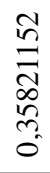 & 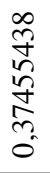 & 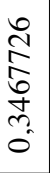 & 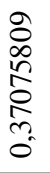 & 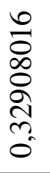 & 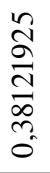 & 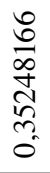 & $\begin{array}{l}\frac{\infty}{\infty} \\
\stackrel{\infty}{\infty} \\
\infty \\
\infty \\
0 \\
0\end{array}$ & 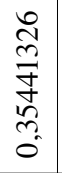 & 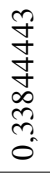 & 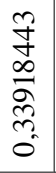 & 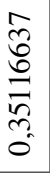 & 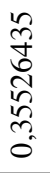 \\
\hline 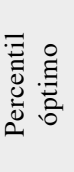 & 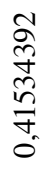 & 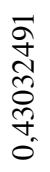 & \begin{tabular}{l}
$\infty$ \\
$\infty$ \\
$\infty$ \\
$\infty$ \\
\multirow{0}{0}{} \\
$\infty$ \\
\multirow{0}{0}{}
\end{tabular} & 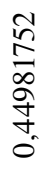 & 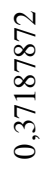 & 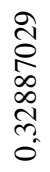 & 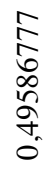 & 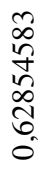 & 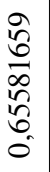 & 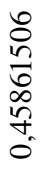 & 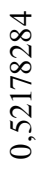 & 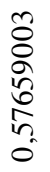 & 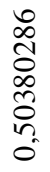 & 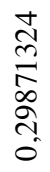 & 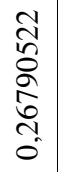 & 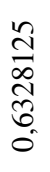 & 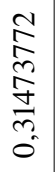 & 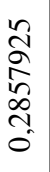 & 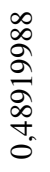 \\
\hline ○. & $\ddot{0}$ & $n$ & $\ddot{0}$ & $\ddot{n}$ & ? & $\tilde{o}$ & $\tilde{o}^{n}$ & "n. & $\tilde{0}$ & $n$ & $\tilde{o}$ & ? & $n$ & $n$ & $\tilde{n}$ & ? & $\tilde{o}$ & $n$ & $\tilde{o}^{n}$ \\
\hline $\begin{array}{l}\text { O } \\
\text { 岁 } \\
\text { \& }\end{array}$ & 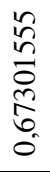 & \begin{tabular}{l}
8 \\
$\stackrel{2}{\hat{n}}$ \\
$\hat{n}$ \\
\multirow{2}{0}{} \\
0
\end{tabular} & 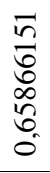 & 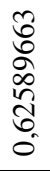 & 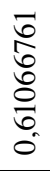 & $\begin{array}{l}\stackrel{8}{2} \\
\hat{2} \\
\infty \\
\text { مे } \\
0\end{array}$ & 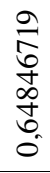 & 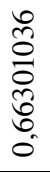 & 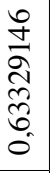 & 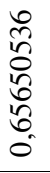 & 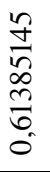 & 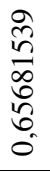 & $\begin{array}{l}\stackrel{n}{N} \\
\stackrel{N}{N} \\
\text { ล̃ } \\
0\end{array}$ & 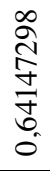 & 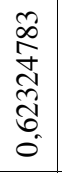 & $\begin{array}{l}\tilde{n} \\
\infty \\
\infty \\
\tilde{n} \\
0 \\
0 \\
0\end{array}$ & $\begin{array}{l}\vec{J} \\
\stackrel{J}{N} \\
\stackrel{J}{0} \\
\stackrel{0}{0} \\
0\end{array}$ & 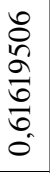 & 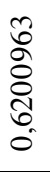 \\
\hline 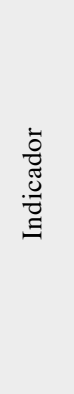 & 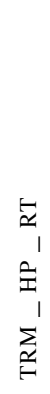 & 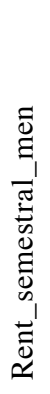 & 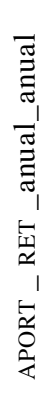 & 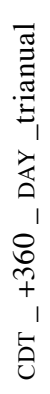 & 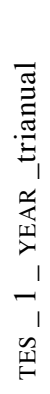 & 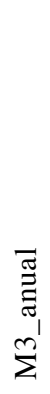 & 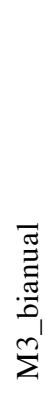 & 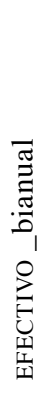 & 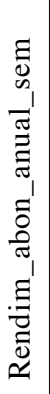 & 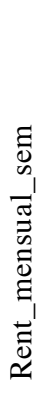 & 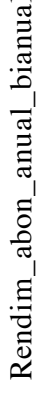 & 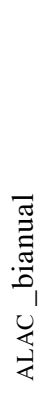 & 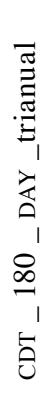 & 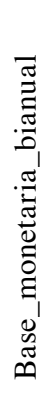 & 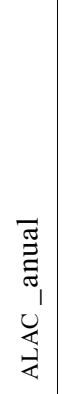 & 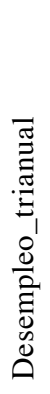 & 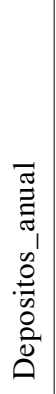 & 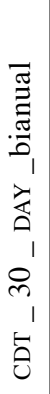 & 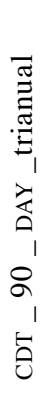 \\
\hline 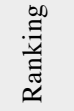 & 2 & ते & $\bar{\sim}$ & $\approx$ & $\tilde{\lambda}$ & $\stackrel{\nabla}{\sim}$ & $\stackrel{2}{a}$ & i & $\hat{\imath}$ & $\stackrel{\infty}{\sim}$ & Әे & లి & $\bar{m}$ & $\approx$ & 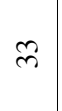 & mे & $\ddot{m}$ & ri & $\hat{m}$ \\
\hline
\end{tabular}




\begin{tabular}{|c|c|c|c|c|c|c|c|c|c|c|c|c|c|c|c|c|c|c|c|}
\hline 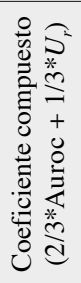 & 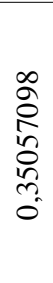 & 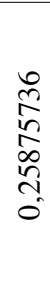 & 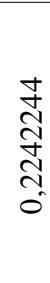 & 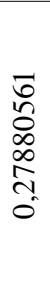 & $\begin{array}{l}\hat{0} \\
\infty \\
0 \\
\infty \\
\tilde{0} \\
\\
0\end{array}$ & $\begin{array}{l}\frac{3}{n} \\
\frac{n}{n} \\
\tilde{n} \\
0\end{array}$ & $\begin{array}{l}\infty \\
\infty \\
\infty \\
\infty \\
\stackrel{+}{+} \\
0\end{array}$ & 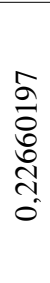 & 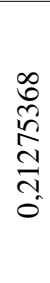 & \begin{tabular}{l}
0 \\
$\infty$ \\
$\infty$ \\
2 \\
$\infty$ \\
\multirow{\forall}{*}{} \\
0 \\
0
\end{tabular} & 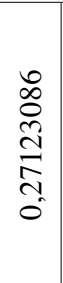 & 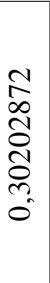 & 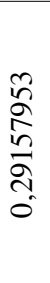 & $\frac{\hat{\tilde{n}}}{\hat{0}}$ & $\begin{array}{l}n \\
\infty \\
\frac{2}{2} \\
\hat{n} \\
\vdots \\
0\end{array}$ & 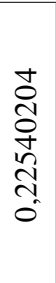 & 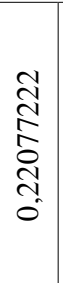 & 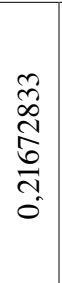 & 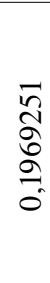 \\
\hline 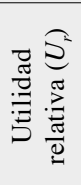 & 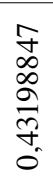 & $\begin{array}{l}n \\
\infty \\
\infty \\
\tilde{n} \\
\tilde{\delta} \\
+ \\
0 \\
0\end{array}$ & $\begin{array}{l}\vec{J} \\
\hat{\sigma} \\
\text { Oे } \\
\text { o. } \\
0\end{array}$ & \begin{tabular}{l}
$\vec{\delta}$ \\
$\infty$ \\
\multirow{+}{*}{} \\
$\vec{J}_{0}$ \\
0
\end{tabular} & $\begin{array}{l}\vec{m} \\
\vec{\nabla} \\
\infty \\
\Xi \\
0\end{array}$ & 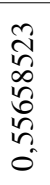 & 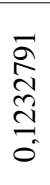 & 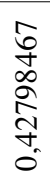 & 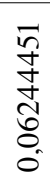 & $\begin{array}{l}n \\
n \\
\infty \\
\infty \\
n \\
n \\
i \\
0\end{array}$ & 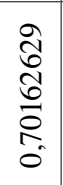 & 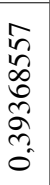 & 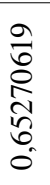 & 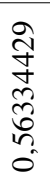 & 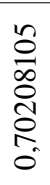 & $\begin{array}{l}\vec{n} \\
\frac{n}{5} \\
\stackrel{0}{0} \\
0\end{array}$ & $\begin{array}{l}\frac{2}{\stackrel{5}{0}} \\
\frac{0}{0} \\
\stackrel{0}{0}\end{array}$ & 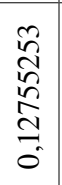 & 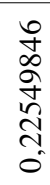 \\
\hline 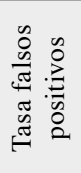 & 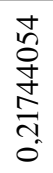 & 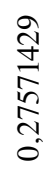 & $\begin{array}{l}\frac{9}{7} \\
0 \\
0 \\
0 \\
0 \\
0\end{array}$ & 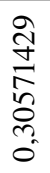 & 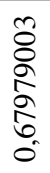 & 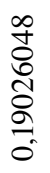 & 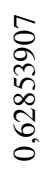 & $\begin{array}{l}0 \\
m \\
m \\
\vec{J} \\
\dot{m} \\
m \\
0\end{array}$ & 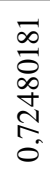 & 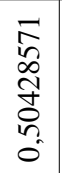 & \begin{tabular}{l}
0 \\
$\infty$ \\
\multirow{J}{J}{} \\
$\underset{\widehat{N}}{\Delta}$ \\
0 \\
0
\end{tabular} & 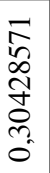 & 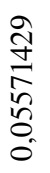 & 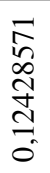 & $\underset{0}{\stackrel{O}{0}}$ & 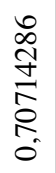 & $\begin{array}{l}\stackrel{2}{0} \\
\stackrel{0}{0} \\
\frac{\infty}{0} \\
0 \\
0\end{array}$ & $\begin{array}{l}\frac{\nabla}{2} \\
\frac{1}{\sqrt{n}} \\
\tilde{\sigma} \\
0 \\
0\end{array}$ & 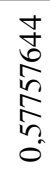 \\
\hline 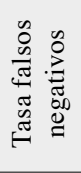 & 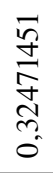 & 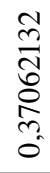 & $\begin{array}{l}\infty \\
\infty \\
\infty \\
\infty \\
\infty \\
\vdots \\
0\end{array}$ & 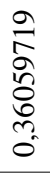 & 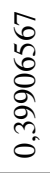 & 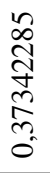 & 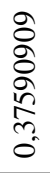 & $\begin{array}{l}\bar{\delta} \\
\circ \\
\stackrel{\circ}{0} \\
\infty \\
\text { ?. } \\
0\end{array}$ & 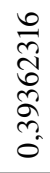 & 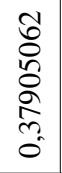 & $\begin{array}{l}\hat{n} \\
\infty \\
\infty \\
\tilde{n} \\
0 \\
0 \\
0 \\
0\end{array}$ & 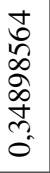 & 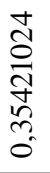 & $\begin{array}{l}n \\
\infty \\
\stackrel{n}{0} \\
m \\
0\end{array}$ & 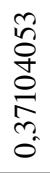 & 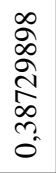 & 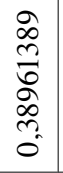 & 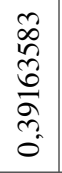 & 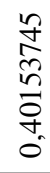 \\
\hline 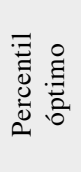 & 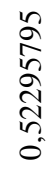 & 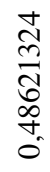 & 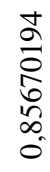 & 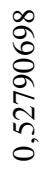 & 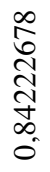 & 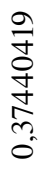 & 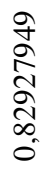 & 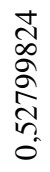 & 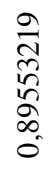 & 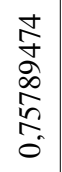 & 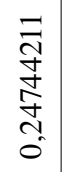 & 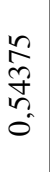 & 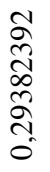 & 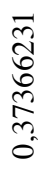 & 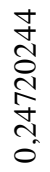 & 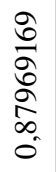 & $\begin{array}{l}\vec{n} \\
\tilde{n} \\
\approx \\
\vdots \\
0\end{array}$ & 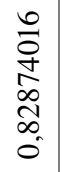 & 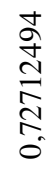 \\
\hline ○. & $\tilde{o}^{n}$ & ñ & $\tilde{n}$ & $\tilde{0}$ & $\ddot{n}$ & $\tilde{o}$ & $\tilde{o}$ & $\tilde{n}_{0}^{n}$ & $\tilde{n}^{n}$ & $\tilde{o}$ & $\tilde{o}$ & $n$ & 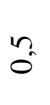 & $\tilde{0}$ & $\tilde{n}$ & $\ddot{n}$ & $\tilde{n}^{n}$ & ? & $\ddot{0}$ \\
\hline 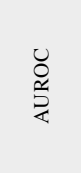 & $\begin{array}{l}\text { तె } \\
2 \\
2 \\
\infty \\
\infty \\
2 \\
0\end{array}$ & 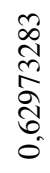 & 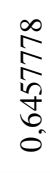 & 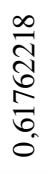 & 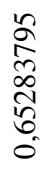 & 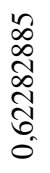 & 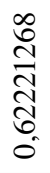 & $\begin{array}{l}\bar{\sigma} \\
\infty \\
\infty \\
\infty \\
\widehat{0} \\
0 \\
0\end{array}$ & $\begin{array}{l}\infty \\
\hat{0} \\
\infty \\
0 \\
0 \\
0\end{array}$ & 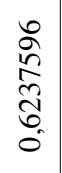 & $\begin{array}{l}\infty \\
\stackrel{\infty}{n} \\
\hat{\sigma} \\
\delta \\
0 \\
0 \\
0\end{array}$ & 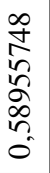 & $\begin{array}{l}0 \\
+ \\
5 \\
\infty \\
\approx \\
\tilde{n} \\
0 \\
0\end{array}$ & 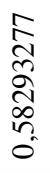 & 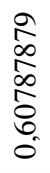 & 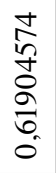 & $\begin{array}{l}\infty \\
\stackrel{8}{0} \\
0 \\
0 \\
0 \\
0 \\
0\end{array}$ & $\begin{array}{l}\frac{n}{n} \\
\frac{n}{2} \\
\frac{n}{2} \\
\sigma \\
0\end{array}$ & 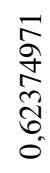 \\
\hline 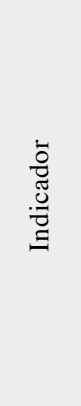 & 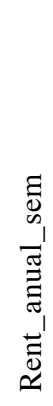 & 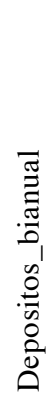 & 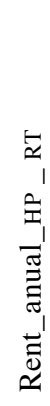 & 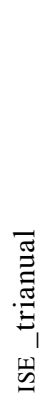 & 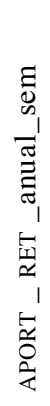 & 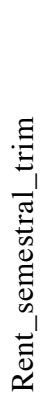 & 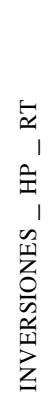 & 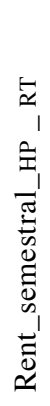 & 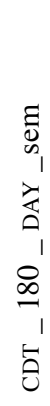 & 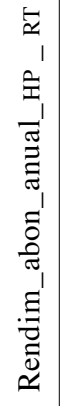 & 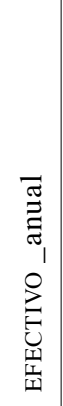 & 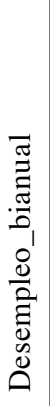 & 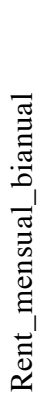 & 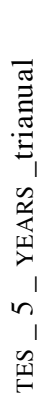 & 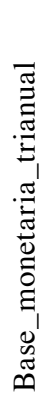 & 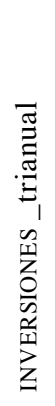 & 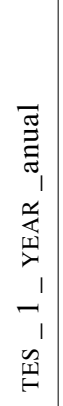 & 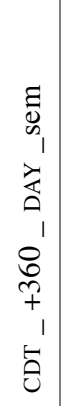 & 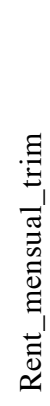 \\
\hline 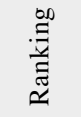 & $\stackrel{\infty}{m}$ & ले & fo & $F$ & F & $\stackrel{F}{f}$ & $\underset{\forall}{\forall}$ & f & fo & F & $\stackrel{\infty}{+}$ & 字 & in & $\bar{n}$ & ก & $\tilde{n}$ & in & $n$ & in \\
\hline
\end{tabular}




\begin{tabular}{|c|c|c|c|c|c|c|c|c|c|c|c|c|c|c|c|c|c|c|c|}
\hline 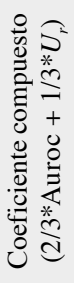 & 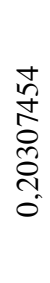 & 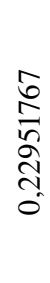 & 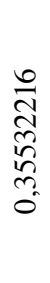 & 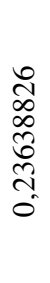 & 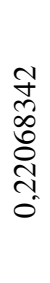 & 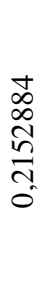 & 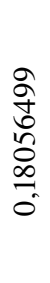 & 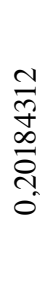 & 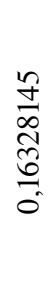 & $\begin{array}{l}\frac{n}{3} \\
0 \\
+ \\
\infty \\
0 \\
0\end{array}$ & $\begin{array}{l}\frac{ \pm}{\sigma} \\
\underset{0}{0} \\
0\end{array}$ & 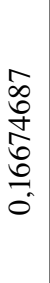 & 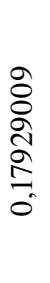 & \begin{tabular}{l}
$\infty$ \\
$\infty$ \\
\multirow{n}{n}{} \\
$\infty$ \\
\multirow{2}{*}{} \\
0
\end{tabular} & 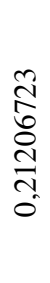 & 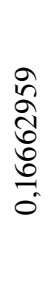 & $\begin{array}{l}n \\
\infty \\
2 \\
\frac{a}{5} \\
\stackrel{5}{0} \\
\stackrel{0}{0}\end{array}$ & 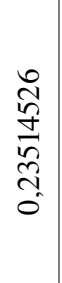 & 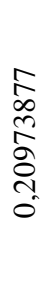 \\
\hline 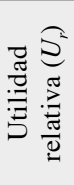 & $\begin{array}{l}\frac{\infty}{\infty} \\
\sqrt[\infty]{n} \\
\infty \\
\cdots \\
0 \\
0\end{array}$ & \begin{tabular}{l}
\multirow{2}{0}{} \\
$\infty$ \\
0 \\
\multirow{2}{*}{} \\
\multirow{2}{n}{} \\
$n$ \\
0
\end{tabular} & 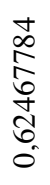 & 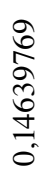 & 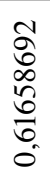 & 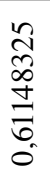 & 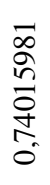 & 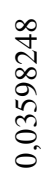 & 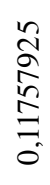 & 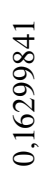 & 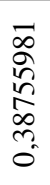 & 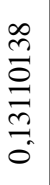 & 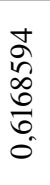 & 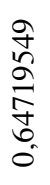 & 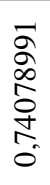 & 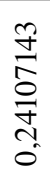 & $\begin{array}{l}\frac{2}{+} \\
\stackrel{+}{0} \\
\stackrel{N}{ \pm} \\
0\end{array}$ & 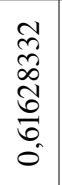 & 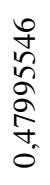 \\
\hline 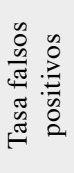 & 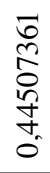 & 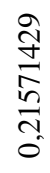 & $\overbrace{0}^{\tilde{O}}$ & 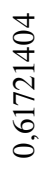 & 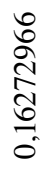 & 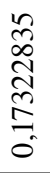 & 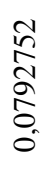 & 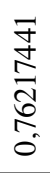 & $\begin{array}{l}\frac{a}{2} \\
\frac{\sigma}{\sigma} \\
\stackrel{0}{\sigma}\end{array}$ & 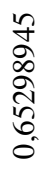 & 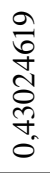 & $\begin{array}{l}\frac{0}{n} \\
\frac{\sqrt{n}}{\hat{\sigma}} \\
\frac{2}{0} \\
0\end{array}$ & 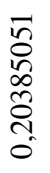 & 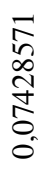 & 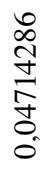 & 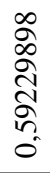 & 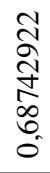 & 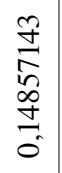 & 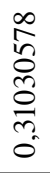 \\
\hline 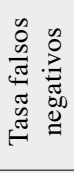 & 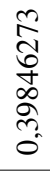 & 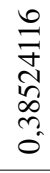 & 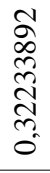 & $\begin{array}{l}\hat{\infty} \\
\infty \\
2 \\
\infty \\
\infty \\
\cdots \\
0 \\
0\end{array}$ & 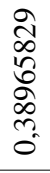 & 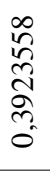 & $\begin{array}{l}\frac{n}{5} \\
\frac{1}{2} \\
\frac{1}{+} \\
0\end{array}$ & 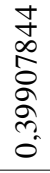 & \begin{tabular}{l}
$\hat{N}$ \\
$\hat{\sigma}$ \\
$\infty$ \\
$\infty$ \\
\multirow{\sigma}{*}{} \\
0
\end{tabular} & 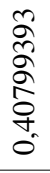 & 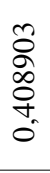 & 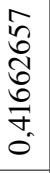 & 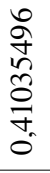 & 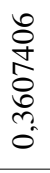 & 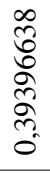 & \begin{tabular}{l}
$\sqrt{n}$ \\
$\infty$ \\
0 \\
0 \\
\multirow{2}{*}{} \\
$\dot{\sigma}$ \\
0
\end{tabular} & 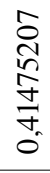 & 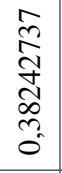 & 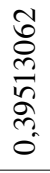 \\
\hline 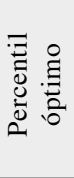 & $\begin{array}{l}n \\
8 \\
8 \\
2 \\
\hat{2} \\
0 \\
0 \\
0\end{array}$ & 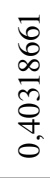 & 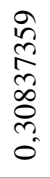 & 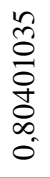 & \begin{tabular}{l} 
oे \\
Oे \\
\multirow{2}{0}{} \\
+े. \\
o.
\end{tabular} & 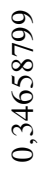 & 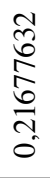 & 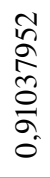 & 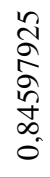 & 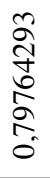 & 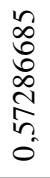 & 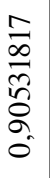 & \begin{tabular}{l}
$\infty$ \\
$\tilde{n}$ \\
$n$ \\
0 \\
\multirow{0}{*}{} \\
$m$ \\
0 \\
0
\end{tabular} & $\begin{array}{l}\text { ․ } \\
\hat{n} \\
\delta \\
\delta \\
0 \\
m \\
0\end{array}$ & 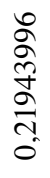 & 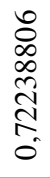 & 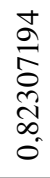 & $\begin{array}{l}\vec{n} \\
0 \\
\delta \\
\delta \\
\infty \\
0 \\
0\end{array}$ & 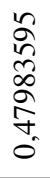 \\
\hline ○. & $\because$ & ñ & $\because$ & $\tilde{0}$ & $\ddot{0}$ & $\tilde{o}$ & $\tilde{0}$ & 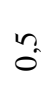 & $\tilde{0}$ & ?n & $\tilde{o}$ & ? & $\tilde{o}$ & $\tilde{o}$ & $\tilde{n}^{n}$ & $\tilde{o}$ & ? & ? & $\tilde{n}$ \\
\hline 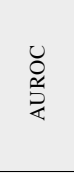 & 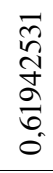 & 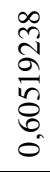 & 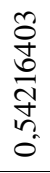 & $\begin{array}{l}\sqrt{2} \\
5 \\
2 \\
2 \\
\tilde{n} \\
0\end{array}$ & $\begin{array}{l}0 \\
\infty \\
\infty \\
\infty \\
\infty \\
\infty \\
\hat{n} \\
0\end{array}$ & 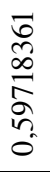 & $\begin{array}{l}\bar{m} \\
\frac{0}{0} \\
\circ \\
0 \\
0 \\
0\end{array}$ & 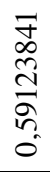 & 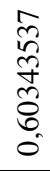 & 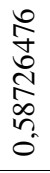 & 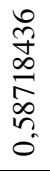 & 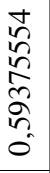 & 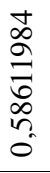 & 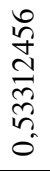 & $\begin{array}{l}0 \\
0 \\
0 \\
0 \\
0 \\
0 \\
0 \\
0\end{array}$ & 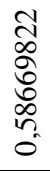 & 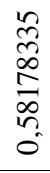 & 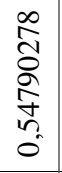 & $\begin{array}{l}\vec{n} \\
\tilde{n} \\
\infty \\
\infty \\
\infty \\
n \\
n \\
0\end{array}$ \\
\hline 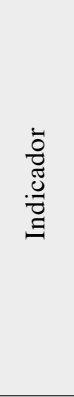 & 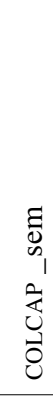 & 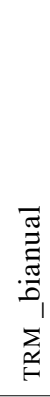 & 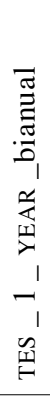 & 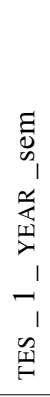 & 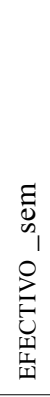 & 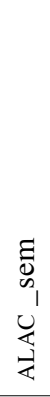 & 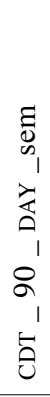 & 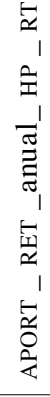 & 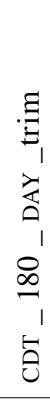 & 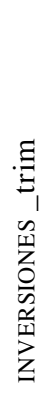 & 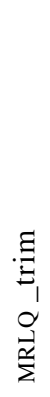 & 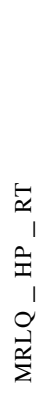 & 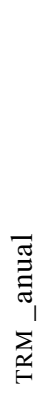 & 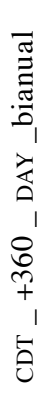 & 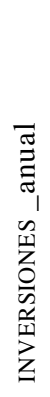 & 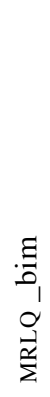 & 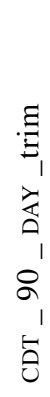 & 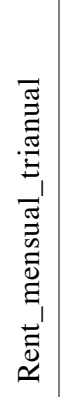 & 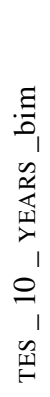 \\
\hline 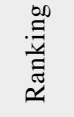 & $\hat{n}$ & in & in & 8 & 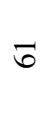 & $\widetilde{\sigma}$ & $\widehat{6}$ & $\vec{b}$ & $\sqrt{6}$ & 8 & $\widehat{\sigma}$ & $\infty$ & g) & 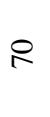 & $\nabla$ & $\mathbb{N}$ & $\stackrel{m}{2}$ & 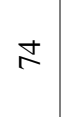 & 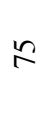 \\
\hline
\end{tabular}




\begin{tabular}{|c|c|c|c|c|c|c|c|c|c|c|c|c|c|c|c|c|c|c|c|}
\hline 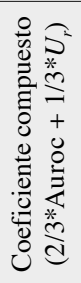 & 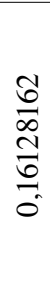 & $\frac{\stackrel{J}{+}}{\stackrel{+}{+}}$ & $\begin{array}{l}\widetilde{N} \\
\tilde{n} \\
2 \\
0 \\
0 \\
\cdots \\
0\end{array}$ & 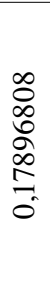 & 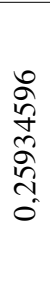 & $\frac{\substack{a \\
\frac{a}{n} \\
\frac{i}{n}}}{0}$ & 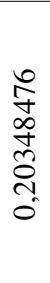 & 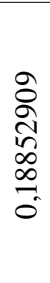 & 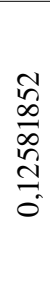 & 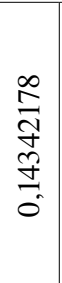 & 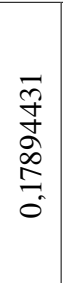 & 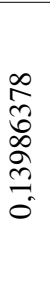 & $\begin{array}{l}n \\
\infty \\
\infty \\
\infty \\
\infty \\
\infty \\
0 \\
0\end{array}$ & 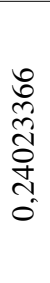 & 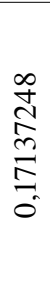 & $\begin{array}{l}\frac{n}{\hat{n}} \\
\frac{a}{5} \\
\frac{a}{n} \\
0\end{array}$ & $\begin{array}{l}\infty \\
\infty \\
\infty \\
\\
心 \\
\vdots \\
0\end{array}$ & $\begin{array}{l}\underset{n}{N} \\
\stackrel{n}{2} \\
\stackrel{a}{a} \\
0\end{array}$ & 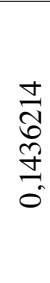 \\
\hline 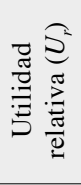 & 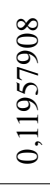 & 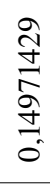 & 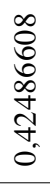 & 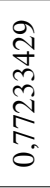 & $\begin{array}{l}\stackrel{0}{2} \\
\text { aे } \\
\frac{a}{2} \\
\stackrel{0}{0}\end{array}$ & 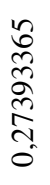 & 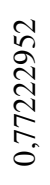 & 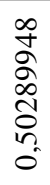 & 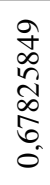 & 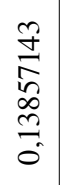 & $\begin{array}{l}\vec{J} \\
\stackrel{\bar{\sigma}}{\sigma} \\
\stackrel{\sigma}{\sigma} \\
\stackrel{0}{0}\end{array}$ & $\begin{array}{l}\bar{Z} \\
0 \\
0 \\
0 \\
0 \\
0 \\
0 \\
0\end{array}$ & 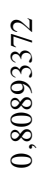 & 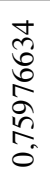 & 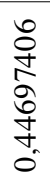 & $\begin{array}{l}\hat{n} \\
\hat{n} \\
\hat{\sigma} \\
\infty \\
+ \\
0 \\
0\end{array}$ & 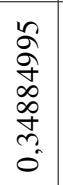 & $\begin{array}{l}\vec{\nabla} \\
\infty \\
\infty \\
\infty \\
\infty \\
+ \\
0\end{array}$ & 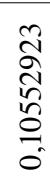 \\
\hline 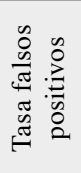 & $\frac{a}{\frac{\partial}{\sigma}}$ & 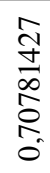 & 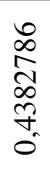 & 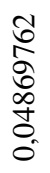 & 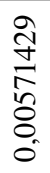 & 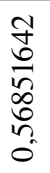 & 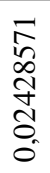 & \begin{tabular}{l}
$\underset{f}{*}$ \\
\multirow{2}{*}{} \\
$\infty$ \\
0 \\
0 \\
0
\end{tabular} & 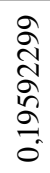 & 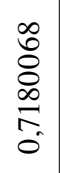 & 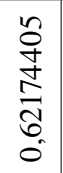 & 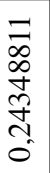 & \begin{tabular}{l}
$\hat{\sigma}$ \\
\multirow{2}{N}{} \\
$\tilde{N}$ \\
$\tilde{0}$ \\
0
\end{tabular} & 0 & 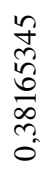 & 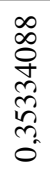 & 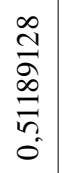 & $\begin{array}{l}0 \\
n \\
\hat{n} \\
\hat{n} \\
0 \\
0 \\
0\end{array}$ & 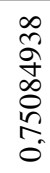 \\
\hline 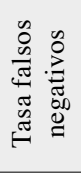 & $\begin{array}{l}\hat{\sigma} \\
\hat{\sigma} \\
\hat{\sigma} \\
\vec{\sigma} \\
0\end{array}$ & 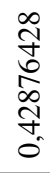 & 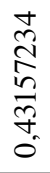 & 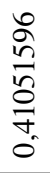 & 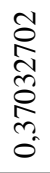 & 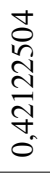 & 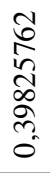 & 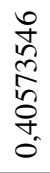 & 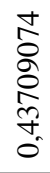 & 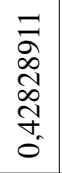 & 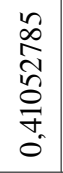 & 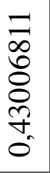 & $\begin{array}{l}\infty \\
\stackrel{0}{0} \\
\infty \\
\stackrel{0}{0} \\
\stackrel{9}{+} \\
0\end{array}$ & $\begin{array}{l}\overrightarrow{\widetilde{N}} \\
\underset{\nabla}{\infty} \\
\infty \\
\stackrel{0}{0} \\
0\end{array}$ & $\begin{array}{l}\stackrel{0}{2} \\
\stackrel{m}{\sigma} \\
\stackrel{m}{*} \\
\underset{\sigma}{\sigma}\end{array}$ & 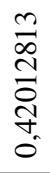 & 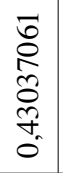 & 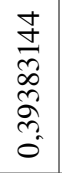 & 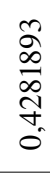 \\
\hline 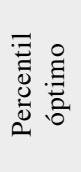 & 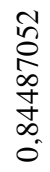 & 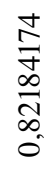 & 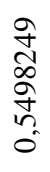 & 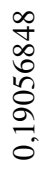 & 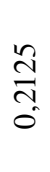 & $\begin{array}{l}\frac{n}{n} \\
\frac{n}{5} \\
\frac{a}{b} \\
0 \\
0\end{array}$ & 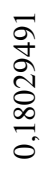 & 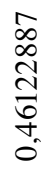 & 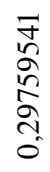 & $\begin{array}{l}\overrightarrow{\widetilde{N}} \\
\overrightarrow{\widetilde{N}} \\
\tilde{N} \\
\infty \\
0 \\
0\end{array}$ & 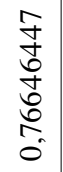 & 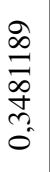 & $\begin{array}{l}\frac{a}{N} \\
\text { तิ } \\
\frac{0}{0}\end{array}$ & $\begin{array}{l}\hat{2} \\
\hat{a} \\
\frac{1}{2} \\
a \\
0\end{array}$ & 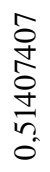 & 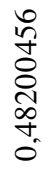 & 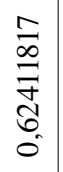 & 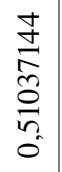 & 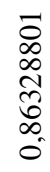 \\
\hline ○. & $\tilde{o}^{n}$ & ñ & $\tilde{0}$ & $\tilde{0}$ & $\tilde{0}$ & $\tilde{o}$ & $\tilde{o}$ & $\tilde{n}_{0}^{n}$ & $\tilde{n}^{n}$ & $\tilde{n}^{n}$ & $\ddot{n}$ & $n$ & 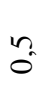 & $\begin{array}{l}\text { ర్ } \\
0\end{array}$ & $\tilde{o}$ & $n$ & $\tilde{0}$ & ? & 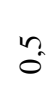 \\
\hline $\begin{array}{l}\text { Oे } \\
\text { జ्य }\end{array}$ & 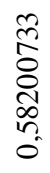 & 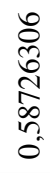 & $\begin{array}{l}\text { to } \\
\stackrel{2}{0} \\
2 \\
\infty \\
\infty \\
n \\
0\end{array}$ & 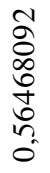 & $\begin{array}{l}\frac{n}{d} \\
\stackrel{2}{5} \\
\stackrel{5}{N} \\
n \\
0\end{array}$ & 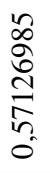 & 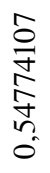 & 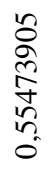 & 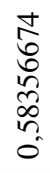 & 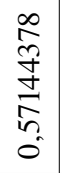 & 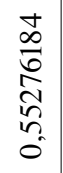 & $\begin{array}{l}\stackrel{\infty}{n} \\
\frac{0}{0} \\
\frac{1}{n} \\
0\end{array}$ & 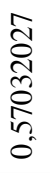 & 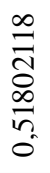 & 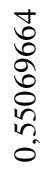 & 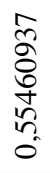 & 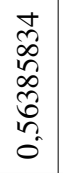 & 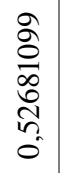 & $\begin{array}{l}\widetilde{b} \\
0 \\
\infty \\
\tilde{n} \\
n \\
n \\
0\end{array}$ \\
\hline 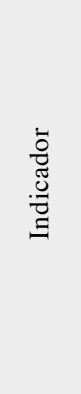 & 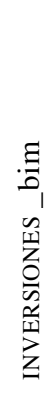 & 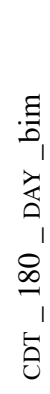 & 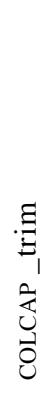 & 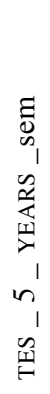 & 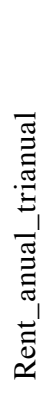 & 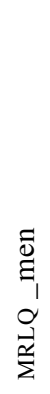 & 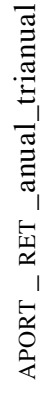 & 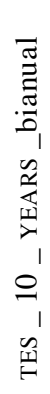 & 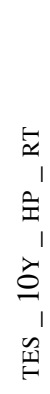 & 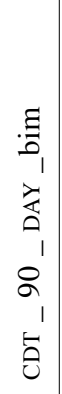 & 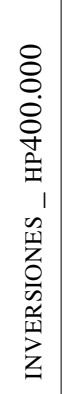 & 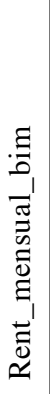 & 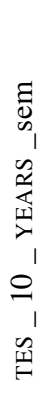 & 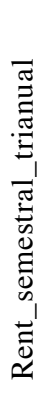 & 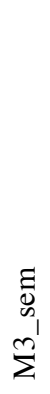 & 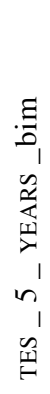 & 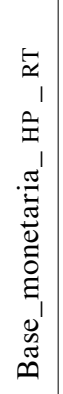 & 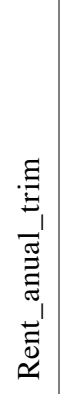 & 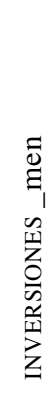 \\
\hline 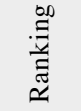 & 2 & 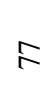 & $\stackrel{\infty}{\sim}$ & 2 & $\infty$ & $\bar{\infty}$ & $\infty$ & $\tilde{\infty}^{0}$ & ఫ & $\mathscr{\infty}$ & $\infty$ & $\infty$ & $\infty$ & ஓे & ฉ̊ & $\bar{\sigma}$ & ๙ু & à & హ゙ \\
\hline
\end{tabular}




\begin{tabular}{|c|c|c|c|c|c|c|c|c|c|c|c|c|c|c|c|c|c|c|c|}
\hline 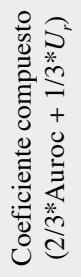 & 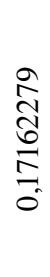 & 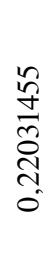 & $\begin{array}{l}\text { I } \\
0 \\
0 \\
0 \\
0 \\
0 \\
0 \\
0\end{array}$ & 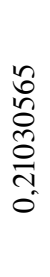 & $\begin{array}{l}\infty \\
0 \\
+ \\
n \\
⿱ 亠 䒑 \\
0 \\
0\end{array}$ & 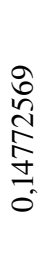 & 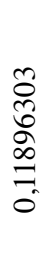 & 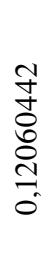 & 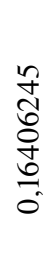 & 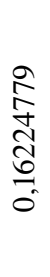 & $\begin{array}{l}\frac{n}{8} \\
\stackrel{J}{7} \\
\stackrel{5}{a} \\
0 \\
0\end{array}$ & 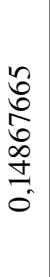 & 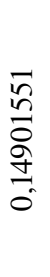 & $\begin{array}{l}\frac{n}{2} \\
\stackrel{5}{0} \\
\stackrel{\Xi}{\Xi} \\
= \\
0\end{array}$ & 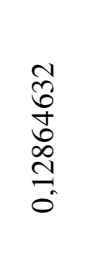 & $\begin{array}{l}\hat{N} \\
\hat{n} \\
0 \\
0 \\
0 \\
0 \\
0\end{array}$ & 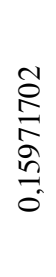 & $\begin{array}{l} \pm \\
n \\
n \\
\infty \\
\infty \\
= \\
0\end{array}$ & 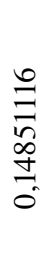 \\
\hline 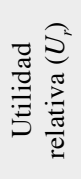 & 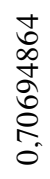 & 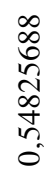 & 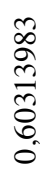 & 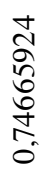 & 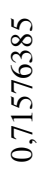 & \begin{tabular}{l}
$m$ \\
\multirow{\sigma}{*}{} \\
$\infty$ \\
$\infty$ \\
$\sigma$ \\
0 \\
0 \\
0
\end{tabular} & 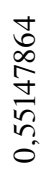 & 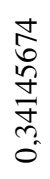 & 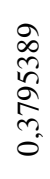 & 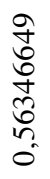 & 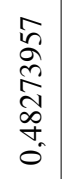 & 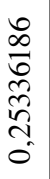 & $\begin{array}{l}1 \\
\delta \\
0 \\
\tilde{n} \\
\text { त̂ } \\
+ \\
0 \\
0\end{array}$ & 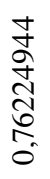 & 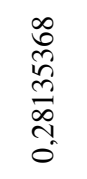 & 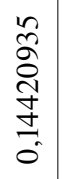 & 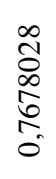 & $\begin{array}{l}\infty \\
\infty \\
\tilde{n} \\
\frac{\pi}{0} \\
\stackrel{0}{0}\end{array}$ & 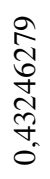 \\
\hline 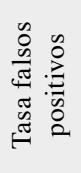 & 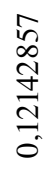 & 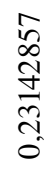 & $\begin{array}{c}\text { त̂ } \\
\tilde{0}\end{array}$ & 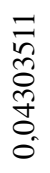 & 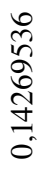 & 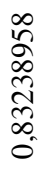 & 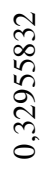 & 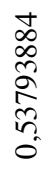 & 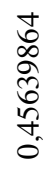 & 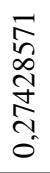 & 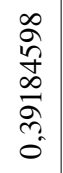 & 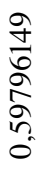 & \begin{tabular}{l}
$f$ \\
+ \\
$\infty$ \\
\multirow{2}{*}{} \\
$\infty$ \\
$\infty$ \\
$\infty$ \\
0
\end{tabular} & 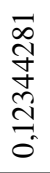 & $\begin{array}{l}\text { ñ } \\
0\end{array}$ & $\begin{array}{l}\frac{0}{\infty} \\
\frac{0}{5} \\
\frac{a}{+} \\
0\end{array}$ & 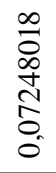 & 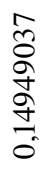 & 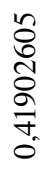 \\
\hline 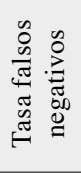 & $\begin{array}{l}\bar{b} \\
\infty \\
\infty \\
\vec{\nabla} \\
\vec{\sigma} \\
0\end{array}$ & 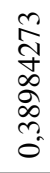 & 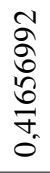 & 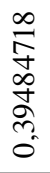 & 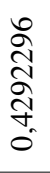 & $\begin{array}{l}\frac{n}{1} \\
\frac{m}{0} \\
\stackrel{2}{+} \\
0 \\
0\end{array}$ & 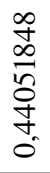 & 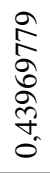 & 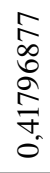 & $\begin{array}{l}\vec{b} \\
\infty \\
\infty \\
\vec{\forall} \\
0\end{array}$ & 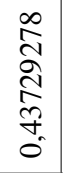 & 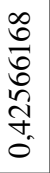 & 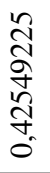 & 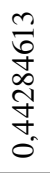 & 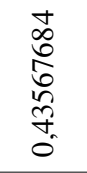 & \begin{tabular}{l}
$=$ \\
$\overrightarrow{0}$ \\
8 \\
0 \\
\multirow{0}{+}{} \\
0 \\
0
\end{tabular} & 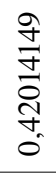 & $\begin{array}{l}\frac{m}{\pi} \\
\hat{n} \\
o \\
\dot{J} \\
0 \\
0\end{array}$ & 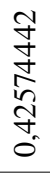 \\
\hline 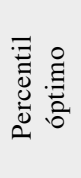 & 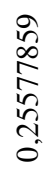 & 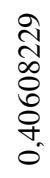 & $\begin{array}{l}3 \\
\infty \\
\infty \\
心 \\
\widetilde{b} \\
\infty \\
0 \\
0\end{array}$ & 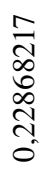 & 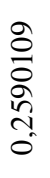 & $\begin{array}{l}\frac{n}{n} \\
\frac{n}{a} \\
\sigma \\
\sigma \\
0\end{array}$ & 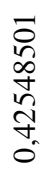 & 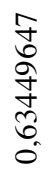 & 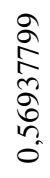 & 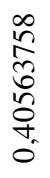 & 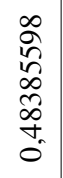 & 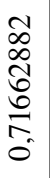 & 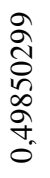 & $\begin{array}{l}\frac{\partial}{\partial} \\
\frac{1}{7} \\
\frac{n}{2} \\
0\end{array}$ & 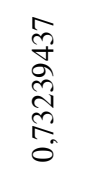 & $\begin{array}{l}\tilde{N} \\
\infty \\
\tilde{N} \\
\tilde{n} \\
\infty \\
\infty \\
0\end{array}$ & 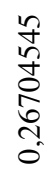 & 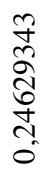 & 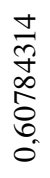 \\
\hline ○. & $\tilde{n}$ & ?n & $\ddot{0}$ & $\tilde{n}^{n}$ & $\ddot{n}$ & $\tilde{o}$ & $\tilde{o}$ & ? & $\tilde{n}^{n}$ & $\tilde{n}^{n}$ & $\ddot{n}$ & $\ddot{n}$ & $\tilde{0}$ & $\tilde{0}$ & $\ddot{n}$ & 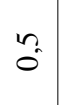 & $n^{n}$ & $\ddot{n}$ & 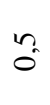 \\
\hline 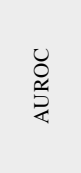 & 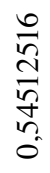 & 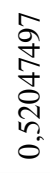 & 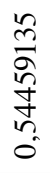 & 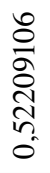 & 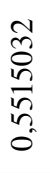 & $\begin{array}{l}\hat{\infty} \\
\infty \\
\\
\infty \\
\dot{\infty} \\
n \\
0\end{array}$ & 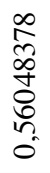 & $\begin{array}{l}\vec{a} \\
\infty \\
+ \\
0 \\
n \\
n \\
0\end{array}$ & 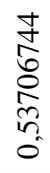 & 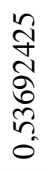 & 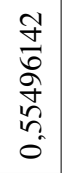 & 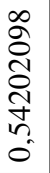 & \begin{tabular}{l}
$\vec{n}$ \\
\multirow{f}{*}{} \\
$n$ \\
$\vec{f}$ \\
$n$ \\
0
\end{tabular} & 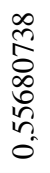 & 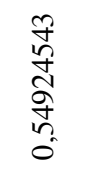 & 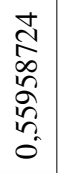 & $\begin{array}{l}n \\
= \\
\infty \\
0 \\
n \\
n \\
0\end{array}$ & 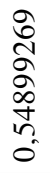 & 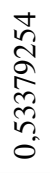 \\
\hline 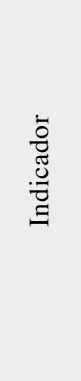 & 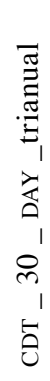 & 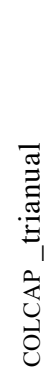 & 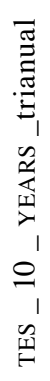 & 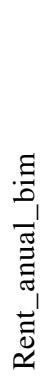 & 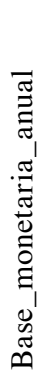 & 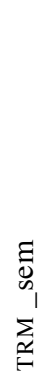 & $\begin{array}{l}\tilde{a} \\
1 \\
\tilde{J}^{\prime} \\
\tilde{n}^{\prime}\end{array}$ & 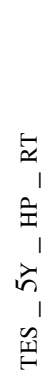 & 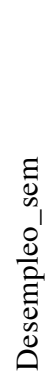 & 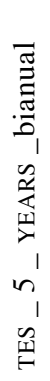 & 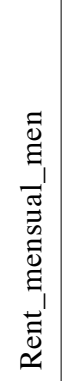 & 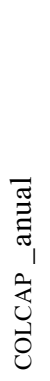 & $\begin{array}{l}\text { हี } \\
\infty \\
\infty \\
0 \\
0 \\
0 \\
0 \\
0 \\
0 \\
0 \\
0\end{array}$ & 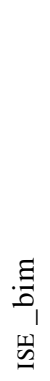 & 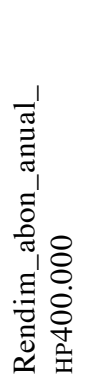 & 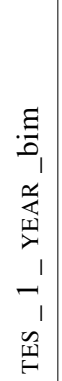 & 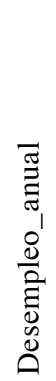 & 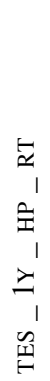 & 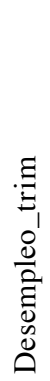 \\
\hline 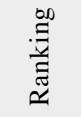 & $\curvearrowleft$ & ஃ & à & 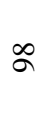 & ন & @ & $\bar{\sigma}$ & తి & $\underline{\varrho}$ & $\underset{0}{ \pm}$ & $\stackrel{?}{\varrho}$ & 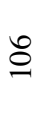 & 으 & $\stackrel{\infty}{\varrho}$ & $\stackrel{\varrho}{ }$ & $\stackrel{\varrho}{ }$ & $\Xi$ & $\stackrel{\cong}{=}$ & $\stackrel{\varrho}{=}$ \\
\hline
\end{tabular}




\begin{tabular}{|c|c|c|c|c|c|c|c|c|c|c|c|c|c|c|c|c|c|c|c|}
\hline 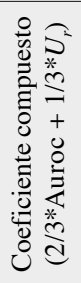 & 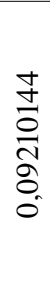 & $\begin{array}{l}\frac{n}{0} \\
0 \\
0 \\
0 \\
0 \\
0\end{array}$ & 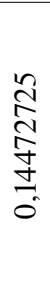 & 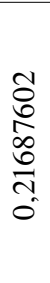 & 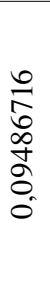 & 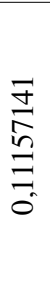 & 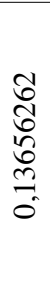 & 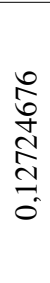 & 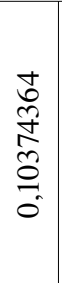 & $\begin{array}{l}\frac{N}{2} \\
\frac{a}{\sigma} \\
0 \\
0\end{array}$ & 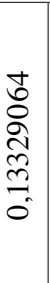 & 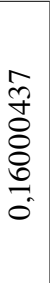 & $\begin{array}{l}\frac{\infty}{n} \\
\frac{1}{n} \\
\infty \\
0 \\
0 \\
0\end{array}$ & 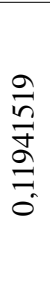 & 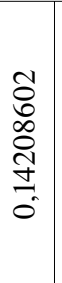 & 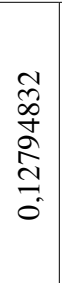 & 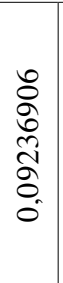 & 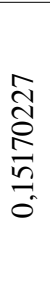 & 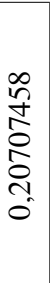 \\
\hline 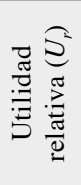 & $\begin{array}{l}\frac{5}{a} \\
\sqrt{2} \\
\hat{n} \\
0 \\
0\end{array}$ & $\begin{array}{l}\vec{J} \\
\stackrel{5}{0} \\
\infty \\
\infty \\
\stackrel{0}{0} \\
0\end{array}$ & 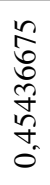 & $\begin{array}{l}2 \\
2 \\
\infty \\
0 \\
0 \\
0 \\
n \\
0\end{array}$ & 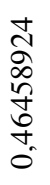 & 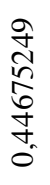 & $\begin{array}{l}\frac{1}{\infty} \\
\infty \\
\tilde{n} \\
\infty \\
\infty \\
0 \\
0\end{array}$ & 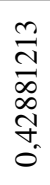 & 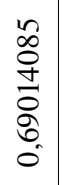 & 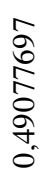 & 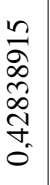 & 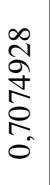 & $\begin{array}{l}\frac{1}{2} \\
\frac{1}{2} \\
\hat{2} \\
\hat{\sigma} \\
0 \\
0\end{array}$ & 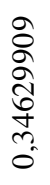 & $\begin{array}{l}\frac{2}{\infty} \\
\frac{\infty}{n} \\
\delta \\
0 \\
0 \\
0\end{array}$ & 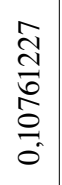 & 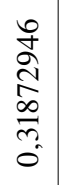 & 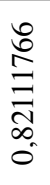 & 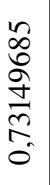 \\
\hline 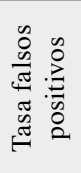 & $\begin{array}{l}\text { مे } \\
\hat{n} \\
\hat{n} \\
\frac{n}{0}\end{array}$ & $\begin{array}{l}\stackrel{0}{0} \\
\infty \\
\infty \\
+ \\
+ \\
+ \\
0\end{array}$ & 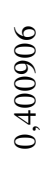 & 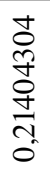 & 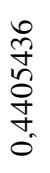 & 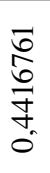 & 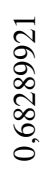 & 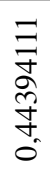 & 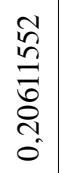 & 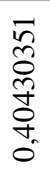 & 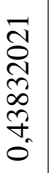 & 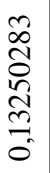 & 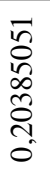 & \begin{tabular}{l}
$\sqrt{n}$ \\
$n$ \\
$\infty$ \\
\multirow{1}{*}{} \\
$\tilde{n}$ \\
$\vdots$ \\
0
\end{tabular} & 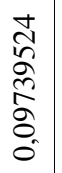 & 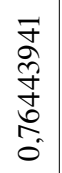 & 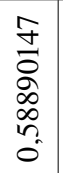 & 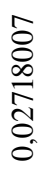 & $\begin{array}{l}\hat{n} \\
\infty \\
\text { I } \\
\text { to } \\
\stackrel{0}{0}\end{array}$ \\
\hline 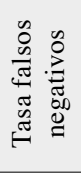 & 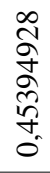 & 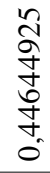 & 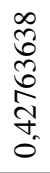 & $\begin{array}{l}\frac{\partial}{\sigma} \\
\frac{0}{n} \\
\frac{\hat{\sigma}}{0} \\
0\end{array}$ & 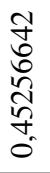 & 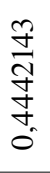 & $\frac{\stackrel{\partial}{\infty}}{\frac{\infty}{\sigma}}$ & 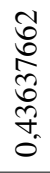 & $\begin{array}{l}\frac{\infty}{\infty} \\
\stackrel{0}{1} \\
\stackrel{\infty}{\infty} \\
\stackrel{+}{+} \\
0\end{array}$ & 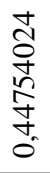 & 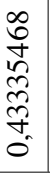 & $\begin{array}{l}\bar{\infty} \\
\hat{\sigma} \\
\sigma \\
\sigma \\
\bar{\sigma} \\
0\end{array}$ & 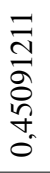 & 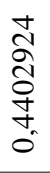 & 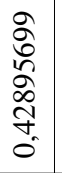 & 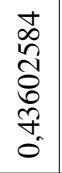 & $\begin{array}{l}\text { fon } \\
\stackrel{n}{\infty} \\
\tilde{n} \\
\tilde{n} \\
\dot{0} \\
0\end{array}$ & 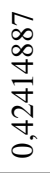 & 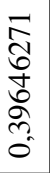 \\
\hline 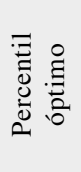 & 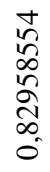 & 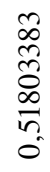 & $\begin{array}{l}2 \\
\hat{n} \\
\tilde{n} \\
\infty \\
\infty \\
\tilde{n} \\
0\end{array}$ & $\begin{array}{l}\frac{\infty}{\infty} \\
\frac{n}{n} \\
\infty \\
\cdots \\
0\end{array}$ & 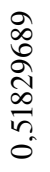 & 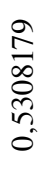 & 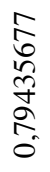 & $\begin{array}{l}\bar{n} \\
0 \\
0 \\
0 \\
1 \\
n \\
0\end{array}$ & $\begin{array}{l}\stackrel{+}{\infty} \\
\stackrel{+}{\sigma} \\
\bar{\sigma} \\
0 \\
0\end{array}$ & 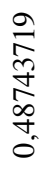 & 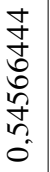 & 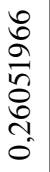 & 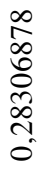 & 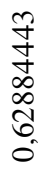 & 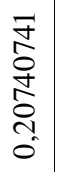 & 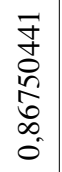 & 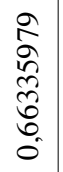 & 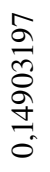 & 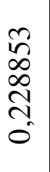 \\
\hline ○. & $\tilde{n}^{n}$ & ñ & $n$ & $\tilde{0}$ & $n$ & $n$ & $\tilde{o}$ & $n$ & $\tilde{n}$ & $n$ & $n$ & $n$ & $\tilde{o}$ & $n$ & ñ & $n$ & $\tilde{o}$ & ? & $n$ \\
\hline $\begin{array}{l}\text { Oे } \\
\text { జ्य }\end{array}$ & $\begin{array}{l}2 \\
\hat{a} \\
+ \\
\infty \\
0 \\
0 \\
0 \\
0\end{array}$ & \begin{tabular}{l}
\multirow{2}{*}{} \\
0 \\
\multirow{+}{*}{} \\
$n$ \\
$n$ \\
0
\end{tabular} & 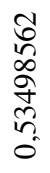 & 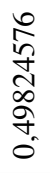 & $\begin{array}{l}0 \\
+ \\
\infty \\
0 \\
\infty \\
n \\
n \\
0 \\
0\end{array}$ & $\begin{array}{l}0 \\
\infty \\
\infty \\
0 \\
\infty \\
\infty \\
1 \\
n \\
0\end{array}$ & 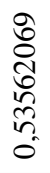 & $\begin{array}{l}n \\
0 \\
\infty \\
0 \\
8 \\
0 \\
\dot{0} \\
n \\
0\end{array}$ & 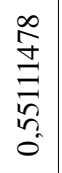 & 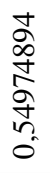 & 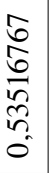 & 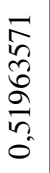 & 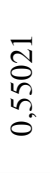 & $\begin{array}{l}\sqrt{0} \\
n \\
2 \\
\infty \\
\infty \\
n \\
0\end{array}$ & $\begin{array}{l}n \\
\infty \\
N \\
N \\
\tilde{N} \\
n \\
0\end{array}$ & $\begin{array}{l}\vec{J} \\
m \\
0 \\
N \\
\tilde{n} \\
0\end{array}$ & 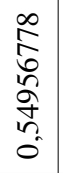 & $\begin{array}{l}\stackrel{\infty}{\Xi} \\
\frac{\infty}{\sigma} \\
\stackrel{\infty}{n} \\
0\end{array}$ & 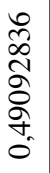 \\
\hline 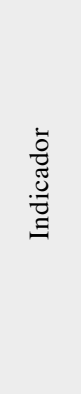 & 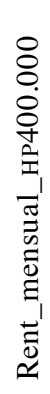 & 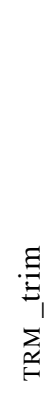 & 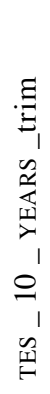 & 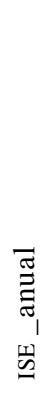 & 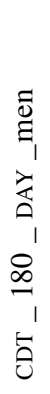 & 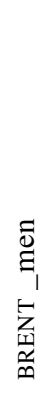 & 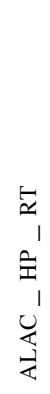 & 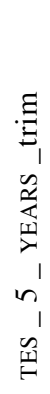 & 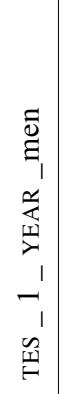 & 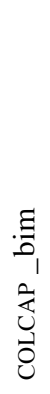 & 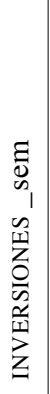 & 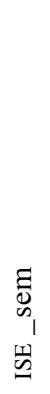 & 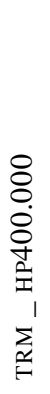 & 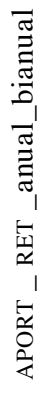 & 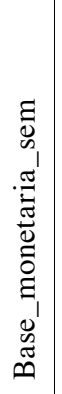 & 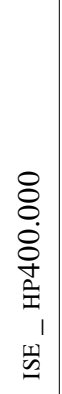 & 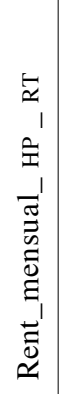 & 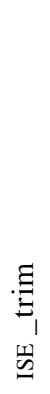 & 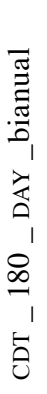 \\
\hline 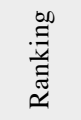 & $\underset{\Xi}{\Xi}$ & $\stackrel{n}{=}$ & $\stackrel{\varrho}{=}$ & 三 & $\stackrel{\infty}{=}$ & $\triangleq$ & さి & $\bar{\Xi}$ & ป & $\cong$ & $\stackrel{\Xi}{\beth}$ & $\stackrel{\beth}{\beth}$ & 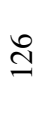 & $\widehat{\cong}$ & $\stackrel{\infty}{\stackrel{\sim}{二}}$ & さे & 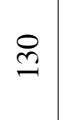 & $\overline{\underline{n}}$ & $\cong$ \\
\hline
\end{tabular}




\begin{tabular}{|c|c|c|c|c|c|c|c|c|c|c|c|c|c|c|c|c|c|c|c|}
\hline 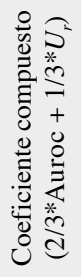 & 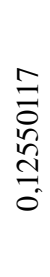 & $\begin{array}{l}\frac{1}{8} \\
\frac{2}{2} \\
\frac{1}{2} \\
0 \\
0\end{array}$ & 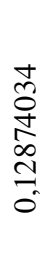 & $\begin{array}{l}\frac{\pi}{\sigma} \\
\frac{\pi}{2} \\
\stackrel{0}{0} \\
0\end{array}$ & 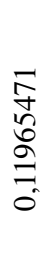 & 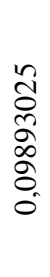 & 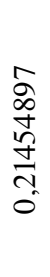 & 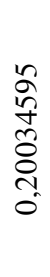 & 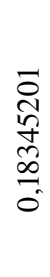 & \begin{tabular}{l}
$\frac{n}{\Xi}$ \\
$\exists$ \\
\multirow{2}{*}{} \\
$\infty$ \\
0 \\
0
\end{tabular} & \begin{tabular}{l}
2 \\
$\infty$ \\
$\infty$ \\
$\infty$ \\
$\infty$ \\
\multirow{2}{0}{} \\
0 \\
0
\end{tabular} & $\begin{array}{l}\hat{b} \\
\infty \\
0 \\
+ \\
+ \\
\infty \\
0 \\
0\end{array}$ & 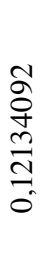 & 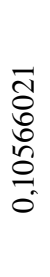 & 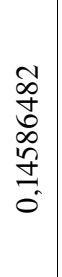 & 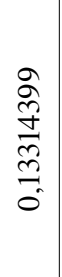 & 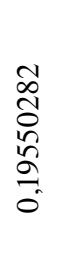 & $\begin{array}{l}\hat{\infty} \\
0 \\
0 \\
\infty \\
⿱ 0 \\
0 \\
0 \\
0\end{array}$ & 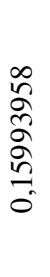 \\
\hline 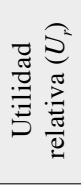 & 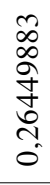 & \begin{tabular}{l}
$\sqrt{0}$ \\
$\infty$ \\
0 \\
\multirow{\sigma}{*}{} \\
0 \\
0
\end{tabular} & $\begin{array}{l}\text { In } \\
0 \\
0 \\
\infty \\
\infty \\
\infty \\
0\end{array}$ & 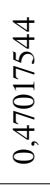 & 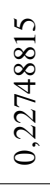 & $\begin{array}{l}\frac{\pi}{n} \\
\frac{n}{2} \\
\delta \\
0 \\
0 \\
0\end{array}$ & $\begin{array}{l}0 \\
0 \\
n \\
\tilde{n} \\
\tilde{n} \\
n \\
0\end{array}$ & 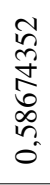 & 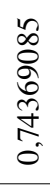 & 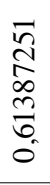 & 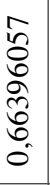 & $\begin{array}{l}\text { సे } \\
\text { مे } \\
0 \\
0 \\
\vdots \\
0 \\
0\end{array}$ & 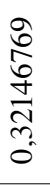 & 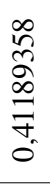 & 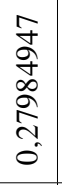 & 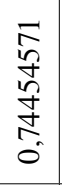 & 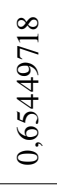 & 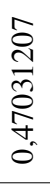 & 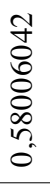 \\
\hline 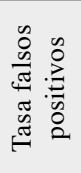 & $\begin{array}{l}\overline{0} \\
0\end{array}$ & 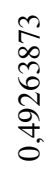 & 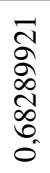 & 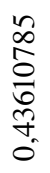 & 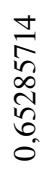 & 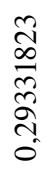 & 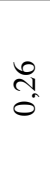 & 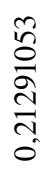 & 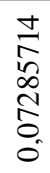 & 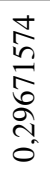 & 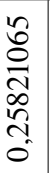 & $\begin{array}{l}\text { Jे } \\
0 \\
\circ \\
\dot{J} \\
\dot{J} \\
0\end{array}$ & \begin{tabular}{l}
$\frac{2}{\sigma}$ \\
$\frac{\sigma}{n}$ \\
\multirow{2}{n}{} \\
0
\end{tabular} & 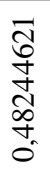 & 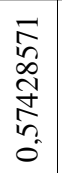 & $\begin{array}{l}\overline{\widehat{\sigma}} \\
\frac{\widehat{N}}{\Delta} \\
\stackrel{0}{0}\end{array}$ & $\frac{n}{0}$ & 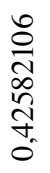 & $\begin{array}{l}\text { İ } \\
\text { ô }\end{array}$ \\
\hline 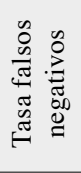 & 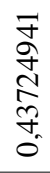 & 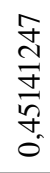 & 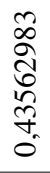 & $\begin{array}{l}\frac{n}{\sigma} \\
\frac{\pi}{\pi} \\
\tilde{n} \\
\stackrel{n}{0}\end{array}$ & 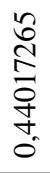 & 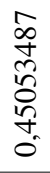 & 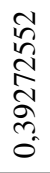 & 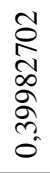 & 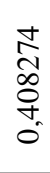 & 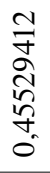 & $\begin{array}{l}\overline{0} \\
\curvearrowleft \\
0 \\
0 \\
0 \\
0 \\
+ \\
0\end{array}$ & 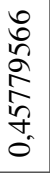 & 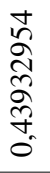 & 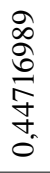 & 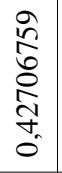 & 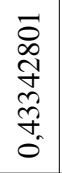 & 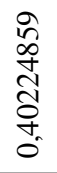 & $\begin{array}{l}\sqrt[n]{0} \\
0 \\
0 \\
\infty \\
\dot{0} \\
\dot{0} \\
0\end{array}$ & 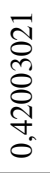 \\
\hline 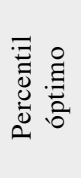 & $\frac{a}{a}$ & 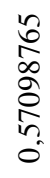 & $\begin{array}{l}\frac{m}{\infty} \\
\stackrel{\infty}{\sigma} \\
\frac{\sigma}{2} \\
0\end{array}$ & 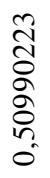 & 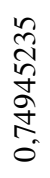 & 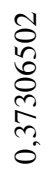 & 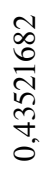 & $\begin{array}{l}\tilde{c} \\
\infty \\
n \\
\hat{n} \\
\infty \\
\infty \\
0 \\
0\end{array}$ & 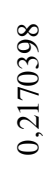 & 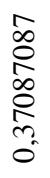 & 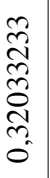 & $\begin{array}{l}\hat{N} \\
\hat{\infty} \\
\infty \\
\\
\infty \\
\infty \\
0 \\
0\end{array}$ & $\begin{array}{l}\frac{n}{2} \\
\frac{n}{n} \\
\frac{n}{2} \\
0 \\
0 \\
0\end{array}$ & 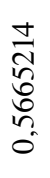 & 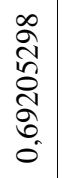 & $\begin{array}{l}\vec{b} \\
\vec{n} \\
0 \\
\tilde{n} \\
0\end{array}$ & \begin{tabular}{l}
0 \\
0 \\
0 \\
0 \\
\multirow{2}{0}{} \\
0 \\
0 \\
0
\end{tabular} & 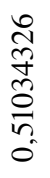 & 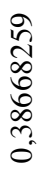 \\
\hline ○. & $\tilde{o}^{n}$ & ñ & $n$ & $\tilde{0}$ & $n$ & $\tilde{o}$ & $\tilde{o}$ & $\tilde{n}_{0}^{n}$ & 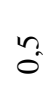 & $n$ & ? & $n$ & 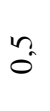 & $n$ & ñ & $\ddot{n}$ & $\ddot{n}$ & ? & $n$ \\
\hline 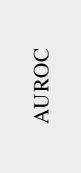 & 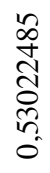 & 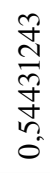 & 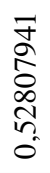 & $\begin{array}{l}\hat{8} \\
\infty \\
o \\
\vec{z} \\
\dot{z} \\
0\end{array}$ & \begin{tabular}{l}
$n$ \\
$\infty$ \\
$\infty$ \\
\multirow{2}{n}{} \\
0 \\
$n$ \\
0 \\
0
\end{tabular} & 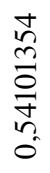 & 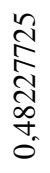 & 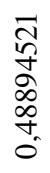 & 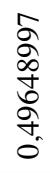 & 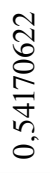 & 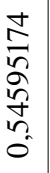 & 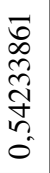 & 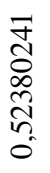 & 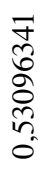 & 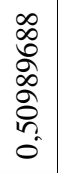 & $\begin{array}{l}\hat{\sigma} \\
\curvearrowleft \\
\infty \\
\infty \\
\stackrel{n}{n} \\
\tilde{n}\end{array}$ & 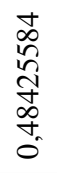 & $\begin{array}{l}\text { 寺 } \\
\hat{\infty} \\
\stackrel{\hat{N}}{ } \\
n \\
0\end{array}$ & $\begin{array}{l}\frac{1}{n} \\
\hat{\sigma} \\
\frac{1}{0} \\
n \\
0\end{array}$ \\
\hline 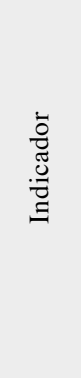 & 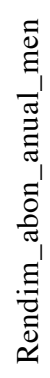 & 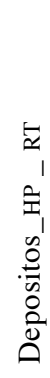 & 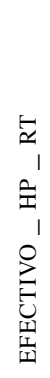 & 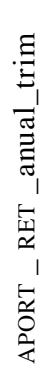 & 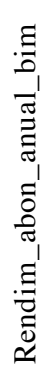 & 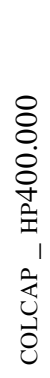 & 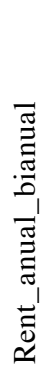 & 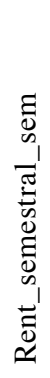 & 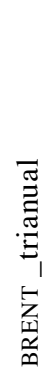 & 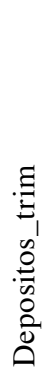 & 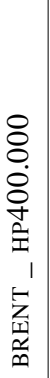 & 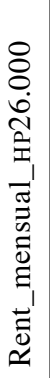 & 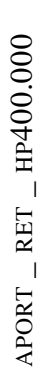 & 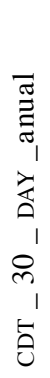 & 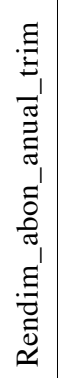 & 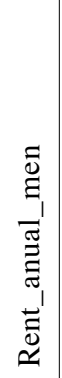 & 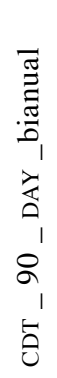 & 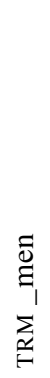 & 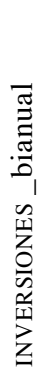 \\
\hline 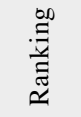 & $\tilde{\Omega}$ & $\stackrel{ \pm}{m}$ & 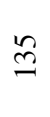 & 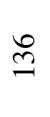 & $\hat{m}$ & $\stackrel{\infty}{\underline{\imath}}$ & ले & $\stackrel{\text { \& }}{\text { I }}$ & $\Xi$ & I & $\stackrel{?}{\Xi}$ & $\underset{J}{ \pm}$ & $\stackrel{\wp}{\exists}$ & $\stackrel{0}{ \pm}$ & 卞 & $\stackrel{\infty}{ \pm}$ & 卞 & $\stackrel{n}{n}$ & $\sqrt{n}$ \\
\hline
\end{tabular}




\begin{tabular}{|c|c|c|c|c|c|c|c|c|c|c|c|c|c|c|c|c|c|c|c|}
\hline 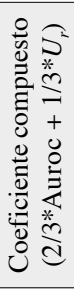 & 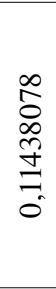 & $\begin{array}{l}\stackrel{2}{\infty} \\
\stackrel{0}{*} \\
\stackrel{2}{0} \\
\stackrel{0}{0} \\
0\end{array}$ & 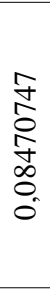 & $\begin{array}{l}\hat{2} \\
\stackrel{2}{\hat{N}} \\
\frac{\hat{N}}{\hat{\delta}} \\
0 \\
0\end{array}$ & 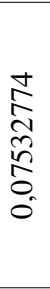 & 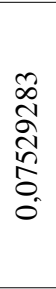 & $\begin{array}{l}\infty \\
\stackrel{2}{1} \\
\curvearrowright \\
\infty \\
\infty \\
0 \\
0\end{array}$ & 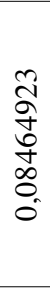 & 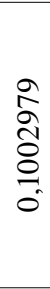 & 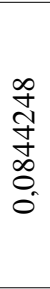 & 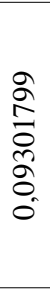 & 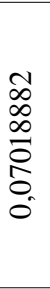 & \begin{tabular}{l}
0 \\
$\infty$ \\
\\
\multirow{2}{n}{} \\
0 \\
0 \\
0
\end{tabular} & 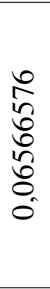 & 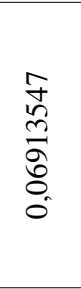 & 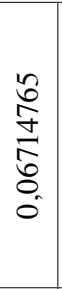 & $\begin{array}{l}\frac{n}{2} \\
\frac{2}{n} \\
\frac{1}{5} \\
0 \\
0\end{array}$ & \begin{tabular}{l}
$\hat{\infty}$ \\
\multirow{2}{\hat{\rho}}{} \\
$\stackrel{0}{0}$ \\
0 \\
0
\end{tabular} & 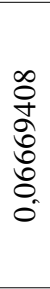 \\
\hline 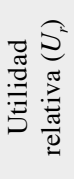 & 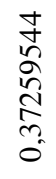 & 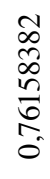 & 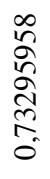 & 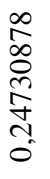 & 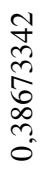 & 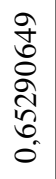 & 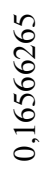 & 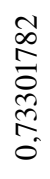 & 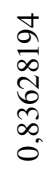 & 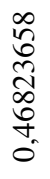 & 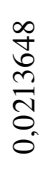 & 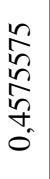 & 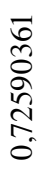 & 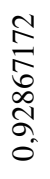 & $\begin{array}{l}\frac{\hat{n}}{\hat{2}} \\
\stackrel{2}{\infty} \\
\hat{\delta} \\
0 \\
0\end{array}$ & 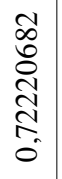 & 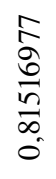 & 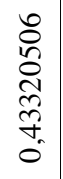 & \begin{tabular}{l}
$\bar{\sigma}$ \\
$\hat{n}$ \\
$o$ \\
0 \\
\multirow{2}{0}{} \\
0
\end{tabular} \\
\hline 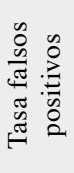 & 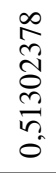 & $\begin{array}{l}\text { है } \\
\text { है } \\
\text { ते } \\
\stackrel{0}{0}\end{array}$ & 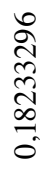 & 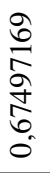 & 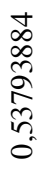 & 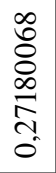 & $\begin{array}{l}\frac{\hat{\sigma}}{\sigma} \\
\frac{\hat{\sigma}}{\hat{0}} \\
\stackrel{0}{0}\end{array}$ & 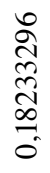 & 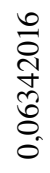 & 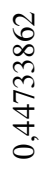 & 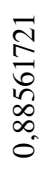 & 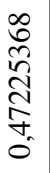 & 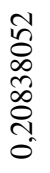 & 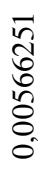 & 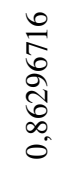 & 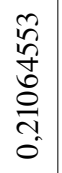 & 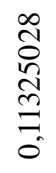 & 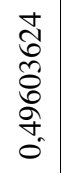 & 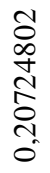 \\
\hline 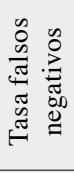 & 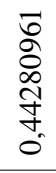 & 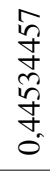 & 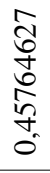 & 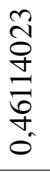 & 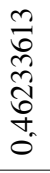 & $\begin{array}{l}\infty \\
\tilde{n} \\
\hat{n} \\
\hat{\delta} \\
\dot{\delta} \\
\dot{0}\end{array}$ & 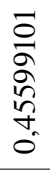 & \begin{tabular}{l} 
aे \\
$\hat{n}$ \\
$\hat{\sigma}$ \\
$\hat{n}$ \\
\multirow{0}{0}{} \\
0
\end{tabular} & 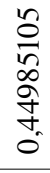 & 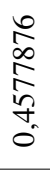 & \begin{tabular}{l}
$\bar{\sigma}$ \\
\multirow{\sigma}{*}{} \\
$\stackrel{n}{\sigma}$ \\
$\dot{\sigma}$
\end{tabular} & 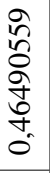 & 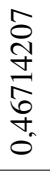 & $\begin{array}{l}\frac{N}{\pi} \\
\frac{1}{6} \\
\delta \\
+ \\
0 \\
0\end{array}$ & 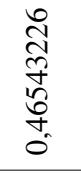 & 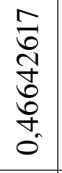 & 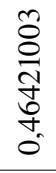 & 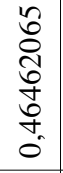 & 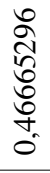 \\
\hline 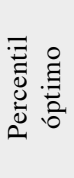 & 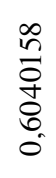 & 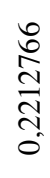 & 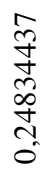 & 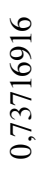 & 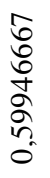 & 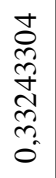 & 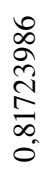 & 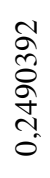 & $\begin{array}{l}\frac{2}{2} \\
\frac{1}{2} \\
\frac{8}{ \pm} \\
0\end{array}$ & $\begin{array}{l}\vec{\sim} \\
\underset{\sim}{\sim} \\
\stackrel{\sim}{n} \\
\tilde{0}\end{array}$ & 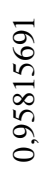 & 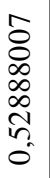 & 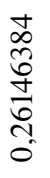 & $\begin{array}{l}\infty \\
\hat{n} \\
\infty \\
\infty \\
0 \\
0 \\
0\end{array}$ & 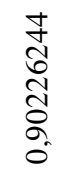 & 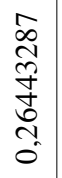 & $\begin{array}{l}\frac{1}{n} \\
\hat{D} \\
\stackrel{n}{5} \\
0 \\
0\end{array}$ & 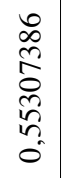 & 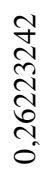 \\
\hline ○. & $\because$ & ñ & ? & $\tilde{o}$ & $\ddot{n}$ & $\tilde{o}$ & $\tilde{o}$ & ? & $\tilde{0}$ & ?n & $\tilde{o}$ & $\tilde{o}$ & $\tilde{o}$ & $\ddot{n}$ & $\tilde{o}^{n}$ & $\ddot{n}$ & $\tilde{n}^{n}$ & $\ddot{n}$ & 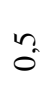 \\
\hline 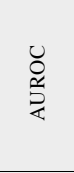 & $\begin{array}{l}\bar{N} \\
\stackrel{N}{N} \\
\tilde{n} \\
0\end{array}$ & 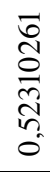 & 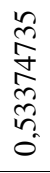 & 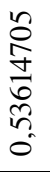 & 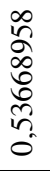 & 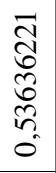 & $\begin{array}{l}0 \\
\cdots \\
\infty \\
\infty \\
\infty \\
n \\
0 \\
0\end{array}$ & 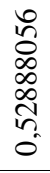 & $\begin{array}{l}\stackrel{+}{n} \\
\frac{a}{+} \\
\infty \\
\tilde{n} \\
0\end{array}$ & $\begin{array}{l}n \\
\infty \\
\infty \\
0 \\
\tilde{n} \\
\vdots \\
0\end{array}$ & 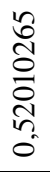 & 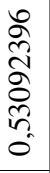 & 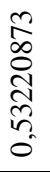 & 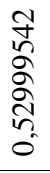 & 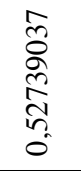 & 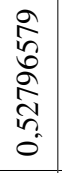 & $\begin{array}{l}\infty \\
\curvearrowright \\
\infty \\
\infty \\
\hat{2} \\
n \\
0\end{array}$ & $\begin{array}{l}0 \\
\widetilde{N} \\
\widetilde{N} \\
\widetilde{N} \\
\tilde{0}\end{array}$ & 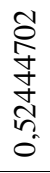 \\
\hline 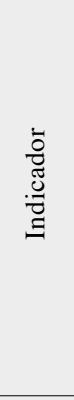 & 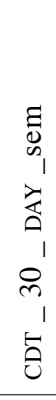 & 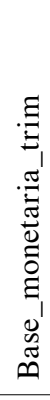 & 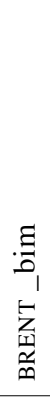 & 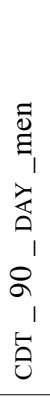 & 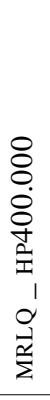 & 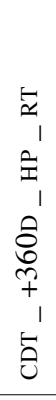 & 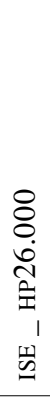 & 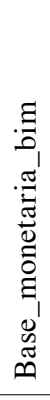 & $\begin{array}{l}\stackrel{\Xi}{\Xi} \\
\Xi \\
\sum^{\prime}\end{array}$ & 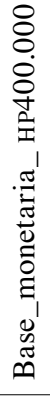 & 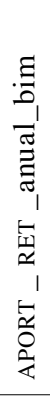 & 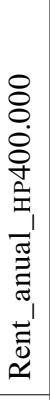 & 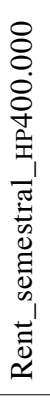 & $\begin{array}{l}\bar{y} \\
1 \\
0 \\
0 \\
1 \\
0 \\
\infty \\
0 \\
1 \\
\overrightarrow{0}\end{array}$ & 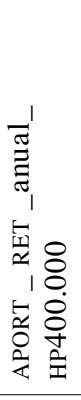 & 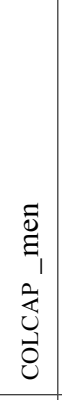 & 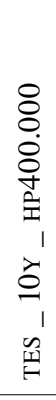 & 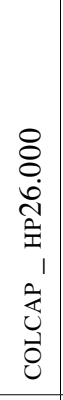 & 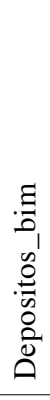 \\
\hline 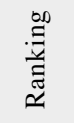 & กี & $\tilde{n}$ & 孚 & $\stackrel{n}{n}$ & 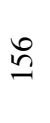 & in & $\stackrel{\infty}{n}$ & $\stackrel{n}{n}$ & $\stackrel{8}{0}$ & $\bar{\sigma}$ & $\widetilde{\sigma}$ & $\underline{6}$ & $\underset{\sigma}{\mathbb{Z}}$ & $\underline{6}$ & $\stackrel{0}{0}$ & $\underline{6}$ & $\stackrel{\infty}{\infty}$ & $\underline{0}$ & 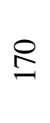 \\
\hline
\end{tabular}




\begin{tabular}{|c|c|c|c|c|c|c|c|c|c|c|c|c|c|c|c|c|c|c|c|}
\hline 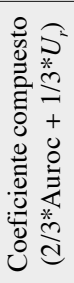 & 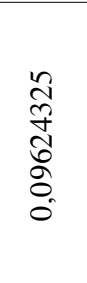 & $\begin{array}{l}0 \\
\stackrel{0}{0} \\
\infty \\
\infty \\
\infty \\
0 \\
0\end{array}$ & $\begin{array}{l}\hat{\varkappa} \\
\tilde{n} \\
\hat{\delta} \\
0 \\
0\end{array}$ & 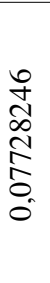 & 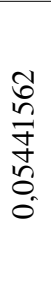 & 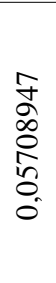 & 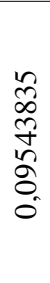 & $\begin{array}{l}\frac{\partial}{\infty} \\
0 \\
+ \\
0 \\
0 \\
0\end{array}$ & 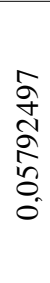 & 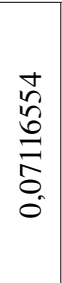 & $\begin{array}{l}\frac{m}{\hat{n}} \\
\infty \\
\overline{0} \\
0 \\
0\end{array}$ & 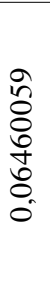 & 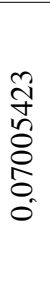 & 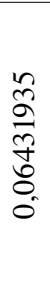 & 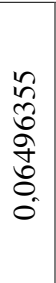 & 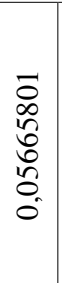 & 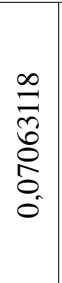 & 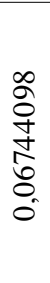 & 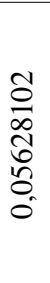 \\
\hline 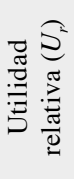 & 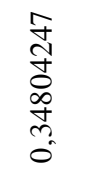 & $\begin{array}{l}0 \\
\infty \\
0 \\
0 \\
\infty \\
\infty \\
\infty \\
0 \\
0 \\
0\end{array}$ & $\begin{array}{l}a \\
\vec{\nabla} \\
\hat{n} \\
\delta \\
0 \\
0 \\
0\end{array}$ & $\begin{array}{l}\frac{\vec{N}}{f} \\
\frac{\mathfrak{d}}{\sigma} \\
\frac{\sigma}{0}\end{array}$ & 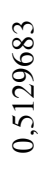 & $\frac{m}{0}$ & 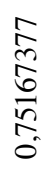 & 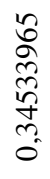 & \begin{tabular}{l}
$\frac{7}{8}$ \\
6 \\
2 \\
\multirow{2}{*}{} \\
0
\end{tabular} & 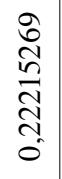 & 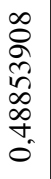 & 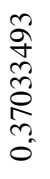 & 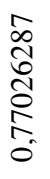 & 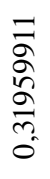 & 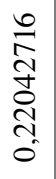 & 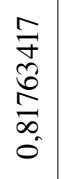 & 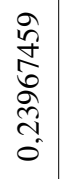 & 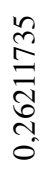 & \begin{tabular}{l}
\multirow{2}{*}{} \\
2 \\
0 \\
$\infty$ \\
$\infty$ \\
0 \\
$n$ \\
0 \\
0
\end{tabular} \\
\hline 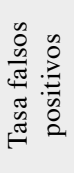 & 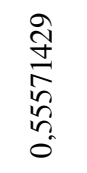 & 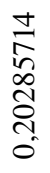 & 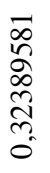 & 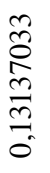 & \begin{tabular}{l}
$\infty$ \\
0 \\
0 \\
0 \\
0 \\
$N$ \\
\multirow{\sigma}{+}{} \\
0
\end{tabular} & 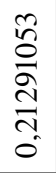 & $\begin{array}{l}\infty \\
\infty \\
\infty \\
\infty \\
\infty \\
\\
0 \\
0\end{array}$ & 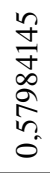 & 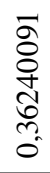 & 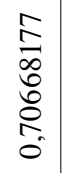 & 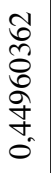 & 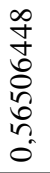 & 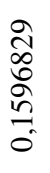 & \begin{tabular}{l}
\multirow{1}{n}{} \\
$\infty$ \\
0 \\
0 \\
0 \\
0 \\
0 \\
0
\end{tabular} & 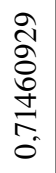 & 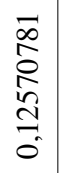 & 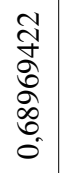 & 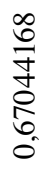 & 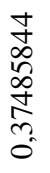 \\
\hline 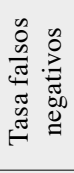 & $\begin{array}{l}\infty \\
\infty \\
\infty \\
\infty \\
\infty \\
\stackrel{\infty}{n} \\
\stackrel{0}{0}\end{array}$ & 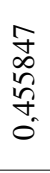 & 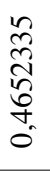 & $\begin{array}{l}\hat{N} \\
\infty \\
\infty \\
\\
\vdots \\
+ \\
+ \\
0\end{array}$ & 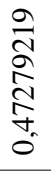 & 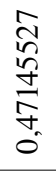 & 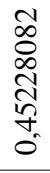 & 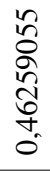 & 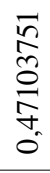 & $\begin{array}{l}\hat{\mathbb{N}} \\
\vec{J} \\
\dot{J} \\
+ \\
\dot{0}\end{array}$ & 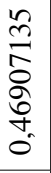 & \begin{tabular}{l}
$\hat{2}$ \\
ôे \\
\multirow{0}{0}{} \\
+ \\
0
\end{tabular} & 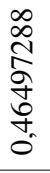 & 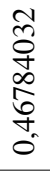 & 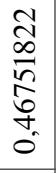 & $\begin{array}{l}\stackrel{2}{8} \\
\hat{\delta} \\
\frac{+}{+} \\
0\end{array}$ & \begin{tabular}{l}
$F$ \\
\multirow{f}{*}{} \\
$\infty$ \\
0 \\
\multirow{0}{0}{} \\
o \\
+ \\
0
\end{tabular} & $\begin{array}{l}\bar{n} \\
\widehat{\hat{~}} \\
\hat{\sigma} \\
0 \\
+ \\
0 \\
0\end{array}$ & 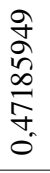 \\
\hline 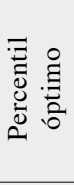 & $\begin{array}{l}\stackrel{2}{N} \\
\stackrel{N}{N} \\
\text { مֶ. } \\
0\end{array}$ & 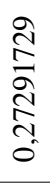 & 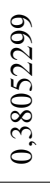 & $\begin{array}{l}\bar{a} \\
\vec{n} \\
\infty \\
\stackrel{0}{\sigma} \\
\overrightarrow{0}\end{array}$ & \begin{tabular}{l} 
के \\
ôे \\
तु \\
\multirow{+}{+}{} \\
o.
\end{tabular} & 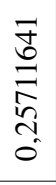 & 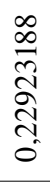 & 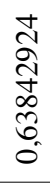 & 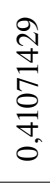 & $\begin{array}{l}\frac{7}{0} \\
\frac{1}{6} \\
\frac{0}{0}\end{array}$ & 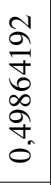 & 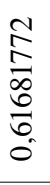 & 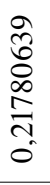 & 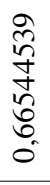 & $\begin{array}{l}\overrightarrow{\tilde{n}} \\
\hat{0} \\
\stackrel{0}{0} \\
\stackrel{0}{0}\end{array}$ & 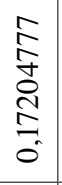 & 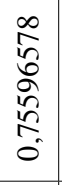 & 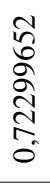 & 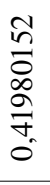 \\
\hline ○. & $\tilde{o}$ & $\tilde{o}$ & $\tilde{o}$ & ? & $\tilde{o}$ & $\tilde{o}$ & $\tilde{o}$ & $\tilde{n}$ & $\tilde{0}$ & $\tilde{o}$ & $\tilde{o}$ & $\tilde{o}$ & $\tilde{o}$ & $\tilde{o}$ & $\tilde{n}^{n}$ & $\ddot{n}$ & $\tilde{n}^{n}$ & $\ddot{n}$ & 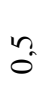 \\
\hline 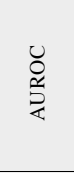 & 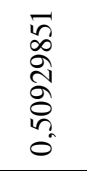 & 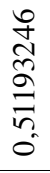 & 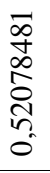 & 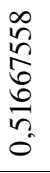 & 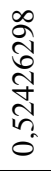 & 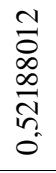 & 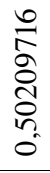 & $\begin{array}{l}\stackrel{n}{+} \\
\stackrel{n}{2} \\
\stackrel{n}{n} \\
0\end{array}$ & $\begin{array}{l}\hat{a} \\
\text { ô } \\
\hat{o} \\
\text { on } \\
\text { o. }\end{array}$ & $\begin{array}{l}n \\
\hat{0} \\
8 \\
0 \\
\frac{1}{n} \\
0 \\
0\end{array}$ & \begin{tabular}{l}
$\stackrel{0}{f}$ \\
\multirow{f}{f}{} \\
$\infty$ \\
$\infty$ \\
\multirow{n}{n}{} \\
0
\end{tabular} & $\begin{array}{l}\stackrel{n}{2} \\
\frac{\tilde{n}}{6} \\
\frac{\sigma}{n} \\
0\end{array}$ & 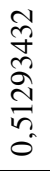 & 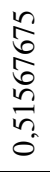 & 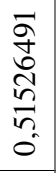 & 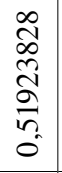 & 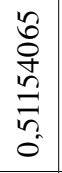 & 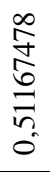 & 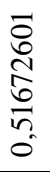 \\
\hline 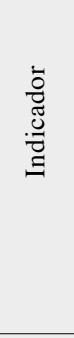 & 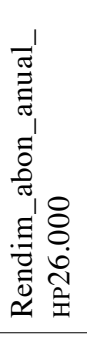 & 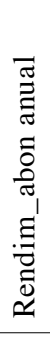 & 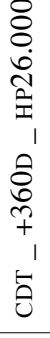 & 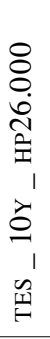 & 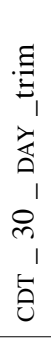 & 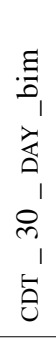 & 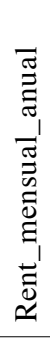 & 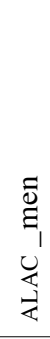 & 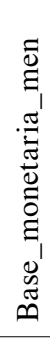 & 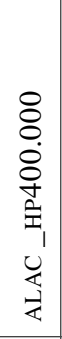 & 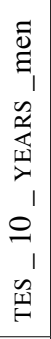 & 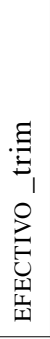 & 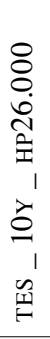 & 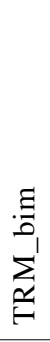 & 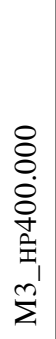 & 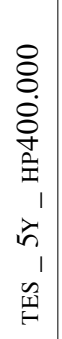 & 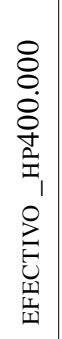 & 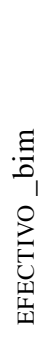 & 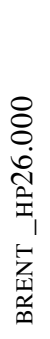 \\
\hline 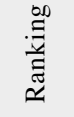 & $\bar{I}$ & $\cong$ & $\stackrel{m}{\Xi}$ & $\stackrel{ \pm}{ \pm}$ & $\stackrel{n}{I}$ & $\stackrel{0}{I}$ & 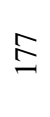 & $\stackrel{\infty}{\beth}$ & 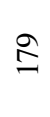 & $\stackrel{\infty}{\simeq}$ & $\underline{\infty}$ & $\stackrel{\infty}{\infty}$ & 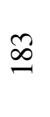 & $\underset{\infty}{\ddagger}$ & 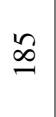 & $\stackrel{\triangleright}{\varnothing}$ & $\stackrel{\infty}{\infty}$ & $\stackrel{\infty}{\infty}$ & $\stackrel{\infty}{\infty}$ \\
\hline
\end{tabular}




\begin{tabular}{|c|c|c|c|c|c|c|c|c|c|c|c|c|c|c|c|c|c|c|c|}
\hline 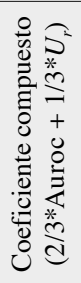 & 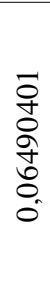 & $\begin{array}{l}\text { त̂ } \\
\text { రి } \\
0 \\
0 \\
0 \\
0 \\
0 \\
0\end{array}$ & \begin{tabular}{l}
\multirow{2}{0}{} \\
$\infty$ \\
0 \\
$\qquad$ \\
0 \\
0 \\
0
\end{tabular} & 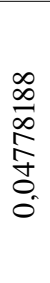 & 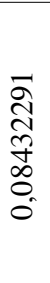 & $\begin{array}{l}\tilde{\infty} \\
\cdots \\
\infty \\
\infty \\
\qquad \\
0 \\
0\end{array}$ & $\begin{array}{l}\stackrel{N}{\cdots} \\
\tilde{0} \\
0 \\
\tilde{n} \\
0 \\
0\end{array}$ & 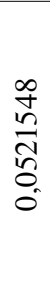 & 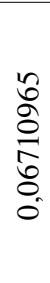 & 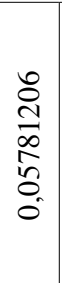 & 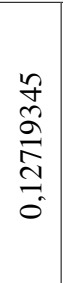 & $\begin{array}{l}\frac{n}{2} \\
\frac{1}{n} \\
\frac{\pi}{n} \\
0 \\
0 \\
0\end{array}$ & $\begin{array}{l}\vec{\delta} \\
8 \\
o \\
\dot{J} \\
\dot{J} \\
0 \\
0\end{array}$ & $\begin{array}{l}2 \\
\infty \\
\infty \\
\infty \\
\hat{n} \\
\hat{0} \\
0\end{array}$ & 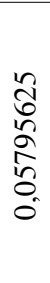 & 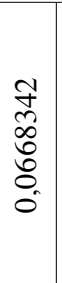 & 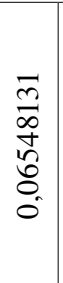 & 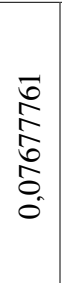 & $\begin{array}{l}\bar{\sigma} \\
\hat{o} \\
0 \\
0 \\
0 \\
0\end{array}$ \\
\hline 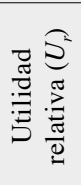 & 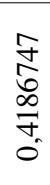 & 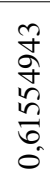 & \begin{tabular}{l}
$\frac{1}{N}$ \\
$\infty$ \\
\multirow{2}{*}{} \\
$\stackrel{2}{2}$ \\
0
\end{tabular} & $\begin{array}{l}\hat{8} \\
8 \\
\infty \\
\frac{0}{2} \\
\stackrel{0}{0}\end{array}$ & $\begin{array}{l}0 \\
+ \\
\infty \\
0 \\
\infty \\
\\
+ \\
0 \\
0\end{array}$ & $\begin{array}{l}0 \\
\stackrel{0}{+} \\
\stackrel{1}{+} \\
0 \\
0\end{array}$ & 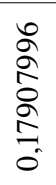 & \begin{tabular}{l}
$\stackrel{0}{2}$ \\
$\frac{1}{0}$ \\
$\vdots$ \\
\multirow{2}{0}{} \\
$\infty$ \\
0
\end{tabular} & 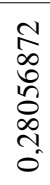 & 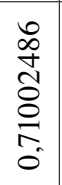 & 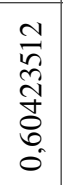 & 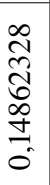 & 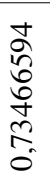 & 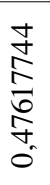 & 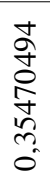 & 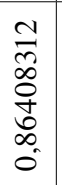 & 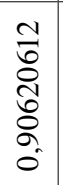 & 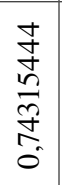 & 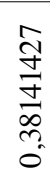 \\
\hline 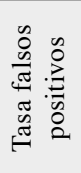 & 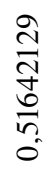 & 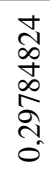 & 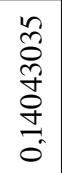 & 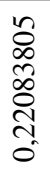 & \begin{tabular}{l}
3 \\
$\infty$ \\
$\infty$ \\
$\infty$ \\
$\ddot{n}$ \\
\multirow{0}{0}{} \\
0
\end{tabular} & $\begin{array}{l}\text { ㄱ. } \\
\text { o } \\
\hat{0} \\
\infty \\
0 \\
0\end{array}$ & 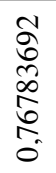 & 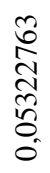 & $\begin{array}{l}\hat{\sigma} \\
\sqrt{\pi} \\
\tilde{2} \\
\tilde{0} \\
0\end{array}$ & 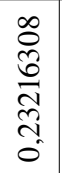 & 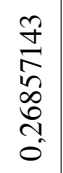 & 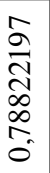 & 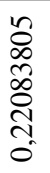 & 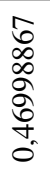 & $\begin{array}{l}\infty \\
\infty \\
m \\
\\
\infty \\
n \\
0\end{array}$ & $\begin{array}{l}\hat{0} \\
\text { o. } \\
0 \\
8 \\
8 \\
0 \\
0\end{array}$ & 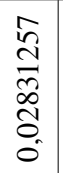 & 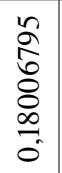 & 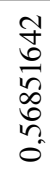 \\
\hline 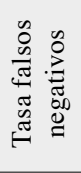 & \begin{tabular}{l}
2 \\
\multirow{2}{+}{} \\
$\frac{1}{6}$ \\
+ \\
0 \\
0
\end{tabular} & $\begin{array}{l}+ \\
\infty \\
\infty \\
0 \\
0 \\
0 \\
\stackrel{0}{0} \\
\stackrel{5}{0} \\
0\end{array}$ & \begin{tabular}{l}
3 \\
\multirow{2}{0}{} \\
$\frac{1}{+}$ \\
\multirow{2}{+}{} \\
0 \\
0
\end{tabular} & 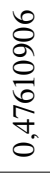 & $\begin{array}{l}n \\
n \\
\infty \\
\infty \\
\infty \\
i n \\
+ \\
0\end{array}$ & 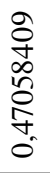 & 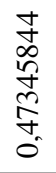 & 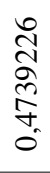 & 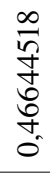 & \begin{tabular}{l}
$\hat{\sigma}$ \\
$\hat{\sigma}$ \\
o \\
\multirow{\sigma}{*}{} \\
$\dot{0}$
\end{tabular} & 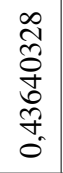 & 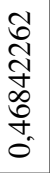 & 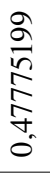 & 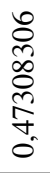 & 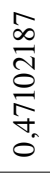 & 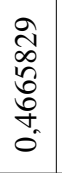 & 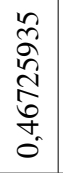 & $\begin{array}{l}0 \\
\Xi \\
0 \\
0 \\
0 \\
0 \\
0\end{array}$ & 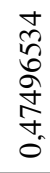 \\
\hline 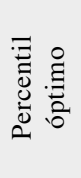 & $\begin{array}{l}\hat{0} \\
\infty \\
\infty \\
\infty \\
\infty \\
0 \\
0 \\
0 \\
0\end{array}$ & 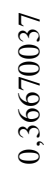 & 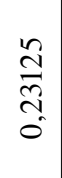 & 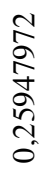 & 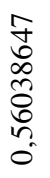 & $\begin{array}{l}n \\
\frac{n}{2} \\
\hat{\delta} \\
\frac{n}{2} \\
a \\
0\end{array}$ & $\begin{array}{l}\bar{\sigma} \\
\text { đె } \\
0 \\
\infty \\
0 \\
0\end{array}$ & 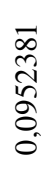 & 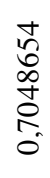 & 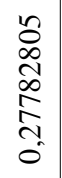 & 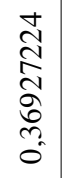 & $\begin{array}{l}\infty \\
0 \\
0 \\
0 \\
0 \\
0 \\
\infty \\
0 \\
0\end{array}$ & 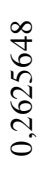 & $\begin{array}{l}\hat{a} \\
\frac{f}{f} \\
\frac{m}{n} \\
0\end{array}$ & 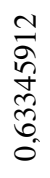 & 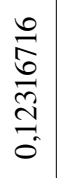 & 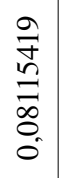 & 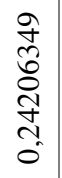 & 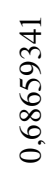 \\
\hline ○. & $\tilde{n}^{n}$ & ñ & $\ddot{0}$ & $\tilde{0}$ & $\ddot{n}$ & $\tilde{o}$ & $\tilde{o}$ & $\tilde{n}_{0}^{n}$ & $\tilde{n}^{n}$ & $n$ & $\ddot{n}$ & $n$ & 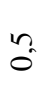 & $\tilde{0}$ & $\tilde{n}$ & $\ddot{n}$ & $\tilde{n}^{n}$ & $\tilde{n}^{n}$ & 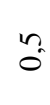 \\
\hline $\begin{array}{l}\text { Oे } \\
\text { జ्य }\end{array}$ & 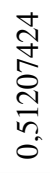 & 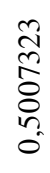 & 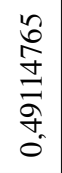 & 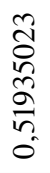 & 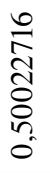 & 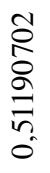 & 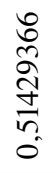 & 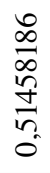 & $\begin{array}{l}8 \\
8 \\
0 \\
0 \\
0 \\
8 \\
0 \\
0\end{array}$ & 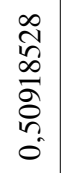 & 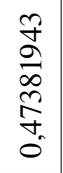 & 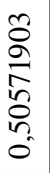 & 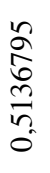 & 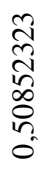 & $\begin{array}{l}\text { Jे } \\
0 \\
0 \\
0 \\
\delta \\
0 \\
0\end{array}$ & \begin{tabular}{l}
$\hat{0}$ \\
$\hat{0}$ \\
\multirow{0}{0}{} \\
0 \\
$\vdots$ \\
0
\end{tabular} & 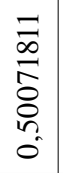 & 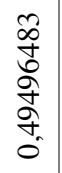 & 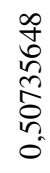 \\
\hline 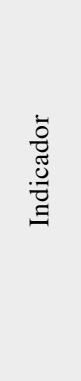 & 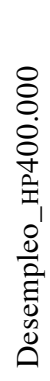 & 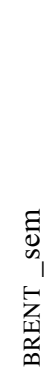 & 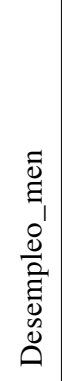 & 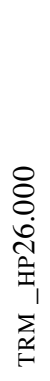 & 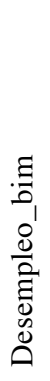 & 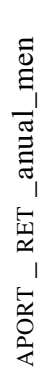 & $\begin{array}{l}8 \\
0 \\
0 \\
0 \\
0 \\
+1 \\
0 \\
0 \\
1 \\
0 \\
0 \\
0 \\
0 \\
0 \\
0 \\
0 \\
0\end{array}$ & 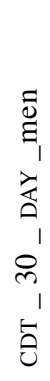 & 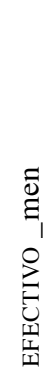 & 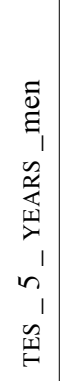 & 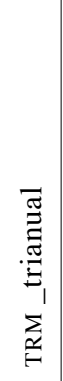 & 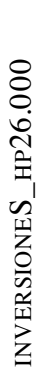 & 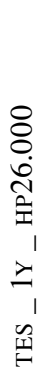 & 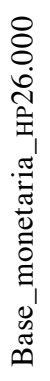 & 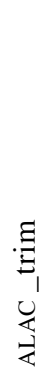 & 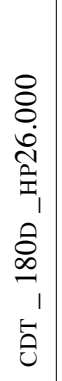 & 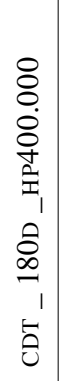 & 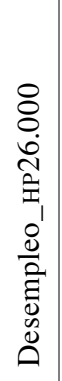 & 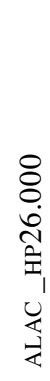 \\
\hline 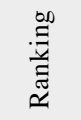 & $\stackrel{8}{\varrho}$ & $\bar{\Xi}$ & ऽ & $\approx$ & 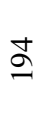 & $\cong$ & $\stackrel{2}{\varrho}$ & $\hat{a}$ & $\approx$ & ळ & 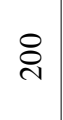 & $\overline{\mathrm{N}}$ & ָิ & હ) & $\underset{\sim}{\stackrel{\overbrace{}}{~}}$ & $\stackrel{n}{\text { ¿n }}$ & 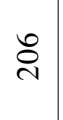 & $\hat{\text { i }}$ & 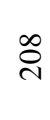 \\
\hline
\end{tabular}




\begin{tabular}{|c|c|c|c|c|c|c|c|c|c|c|c|c|c|c|c|c|c|c|c|}
\hline 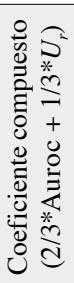 & 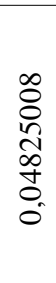 & 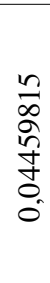 & 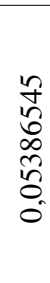 & 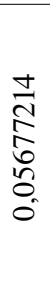 & 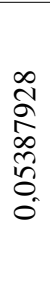 & $\begin{array}{l}n \\
0 \\
\infty \\
\infty \\
\infty \\
0 \\
0 \\
0\end{array}$ & \begin{tabular}{l}
3 \\
$\infty$ \\
$\infty$ \\
$\infty$ \\
\multirow{2}{0}{} \\
0 \\
0 \\
0
\end{tabular} & 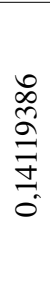 & $\begin{array}{l}\hat{b} \\
\stackrel{2}{0} \\
\hat{n} \\
0 \\
0 \\
0 \\
0\end{array}$ & 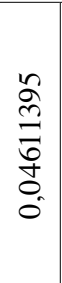 & 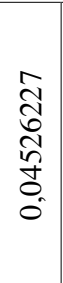 & 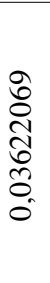 & 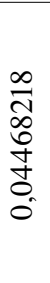 & $\begin{array}{l}\frac{\Omega}{a} \\
\hat{n} \\
0 \\
0 \\
0 \\
0\end{array}$ & 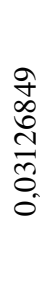 & 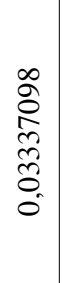 & 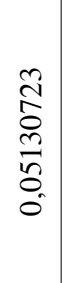 & 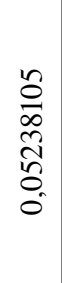 & $\begin{array}{l}\hat{n} \\
\stackrel{n}{N} \\
\hat{n} \\
0 \\
0 \\
0\end{array}$ \\
\hline 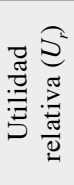 & 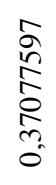 & 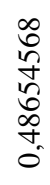 & 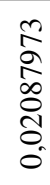 & 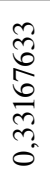 & 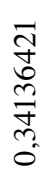 & 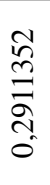 & 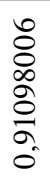 & 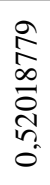 & 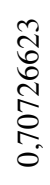 & 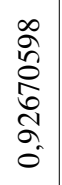 & 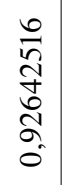 & 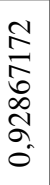 & 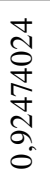 & 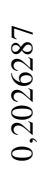 & $\begin{array}{l}\text { ते } \\
\hat{\delta} \\
0 \\
\tilde{0} \\
\hat{0} \\
0\end{array}$ & $\begin{array}{l}n \\
n \\
n \\
0 \\
o \\
0 \\
n \\
n \\
0\end{array}$ & 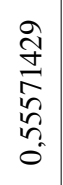 & 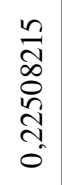 & $\begin{array}{l}\frac{9}{g} \\
\frac{m}{0} \\
\tilde{n} \\
0\end{array}$ \\
\hline 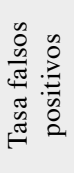 & 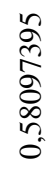 & $\begin{array}{l}\text { Гo } \\
\infty \\
\infty \\
\infty \\
0 \\
+ \\
0 \\
0\end{array}$ & 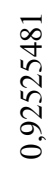 & 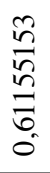 & 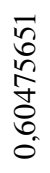 & 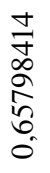 & 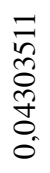 & $\begin{array}{l}n \\
\infty \\
\infty \\
\infty \\
\infty \\
m \\
0 \\
0\end{array}$ & 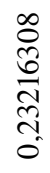 & 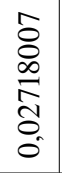 & 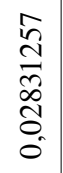 & $\begin{array}{l}\hat{2} \\
\hat{0} \\
\frac{\tilde{n}}{\hat{n}} \\
\hat{0} \\
0\end{array}$ & $\begin{array}{l}\infty \\
\stackrel{n}{\kappa} \\
\hat{n} \\
o \\
⿱ 乛 \\
0 \\
0\end{array}$ & 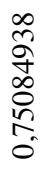 & 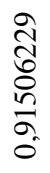 & 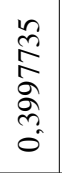 & $\begin{array}{l}\infty \\
\infty \\
\infty \\
\hat{\sigma} \\
\widehat{్} \\
\text { ભે } \\
0\end{array}$ & 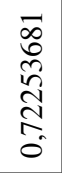 & 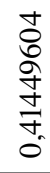 \\
\hline 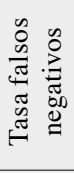 & 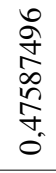 & 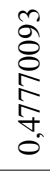 & 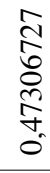 & 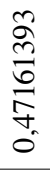 & 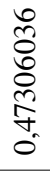 & 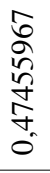 & 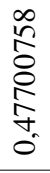 & 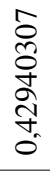 & \begin{tabular}{l} 
o \\
\multirow{2}{+}{} \\
$\hat{\sigma}$ \\
o \\
+ \\
0 \\
0
\end{tabular} & 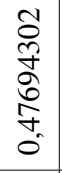 & \begin{tabular}{l}
$\infty$ \\
$\infty$ \\
$\infty$ \\
$\infty$ \\
$\stackrel{2}{+}$ \\
\multirow{+}{*}{} \\
0
\end{tabular} & 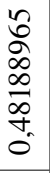 & 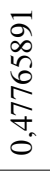 & 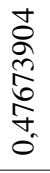 & $\begin{array}{l}n \\
\hat{n} \\
0 \\
⿱ 亠 䒑 \\
+ \\
\infty \\
+ \\
0 \\
0\end{array}$ & $\begin{array}{l}\vec{r} \\
\stackrel{\sigma}{m} \\
\stackrel{\infty}{\infty} \\
\stackrel{\infty}{+}\end{array}$ & 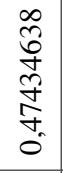 & 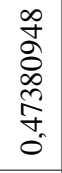 & 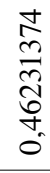 \\
\hline 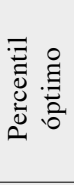 & \begin{tabular}{l}
$\circ$ \\
\multirow{2}{0}{} \\
$\frac{n}{n}$ \\
$\frac{2}{2}$ \\
0
\end{tabular} & 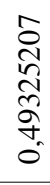 & 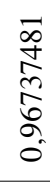 & 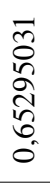 & 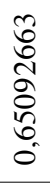 & $\begin{array}{l}2 \\
\bar{\sigma} \\
\hat{0} \\
\hat{\sigma} \\
0 \\
0\end{array}$ & $\begin{array}{l}\overrightarrow{0} \\
\infty \\
0 \\
0 \\
\infty \\
\infty \\
0 \\
0\end{array}$ & 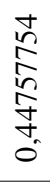 & 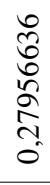 & 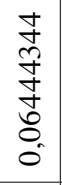 & $\begin{array}{l}\hat{0} \\
\stackrel{0}{J} \\
\infty \\
0 \\
0 \\
0 \\
0\end{array}$ & 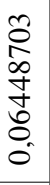 & 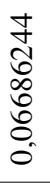 & 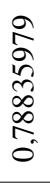 & $\begin{array}{l}m \\
\hat{n} \\
\hat{n} \\
o \\
\vdots \\
0 \\
0\end{array}$ & 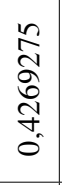 & 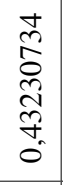 & 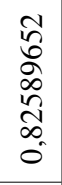 & 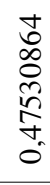 \\
\hline ○. & $\because$ & ñ & $\tilde{0}$ & $\tilde{0}$ & ? & $\tilde{o}$ & $\tilde{o}$ & ? & $\tilde{n}^{n}$ & $\ddot{n}$ & $\ddot{n}$ & ? & $\tilde{o}$ & $\tilde{o}$ & $\tilde{n}^{n}$ & $\tilde{o}^{n}$ & $\tilde{0}$ & $\tilde{n}^{n}$ & ? \\
\hline 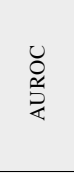 & $\begin{array}{l}\frac{1}{N} \\
\hat{n} \\
\infty \\
\infty \\
0 \\
n \\
0\end{array}$ & 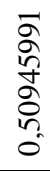 & 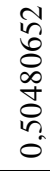 & $\begin{array}{l}\frac{\infty}{N} \\
\frac{\infty}{\infty} \\
\frac{0}{0} \\
\vdots \\
0\end{array}$ & 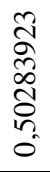 & 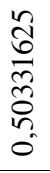 & 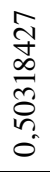 & 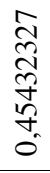 & \begin{tabular}{l}
2 \\
$\hat{\sigma}$ \\
+ \\
\multirow{+}{\alpha}{} \\
\multirow{\sigma}{+}{} \\
0
\end{tabular} & $\begin{array}{c}\infty \\
0 \\
0 \\
\infty \\
0 \\
8 \\
0 \\
n \\
0 \\
0\end{array}$ & 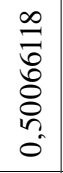 & 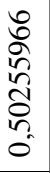 & 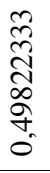 & 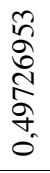 & 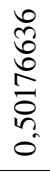 & $\begin{array}{l}\text { ㄹ. } \\
\bar{n} \\
\delta \\
8 \\
0 \\
n \\
0\end{array}$ & $\begin{array}{l}\exists \\
\bar{\sigma} \\
\stackrel{2}{\alpha} \\
\dot{0} \\
\dot{0}\end{array}$ & $\begin{array}{l}\vec{\widetilde{ }} \\
\tilde{\delta} \\
\infty \\
\infty \\
+ \\
+ \\
+\end{array}$ & 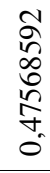 \\
\hline 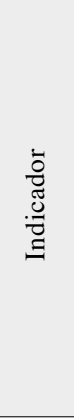 & 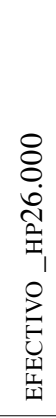 & 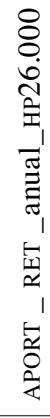 & 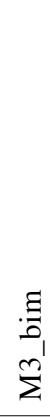 & 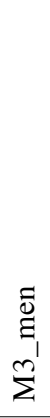 & 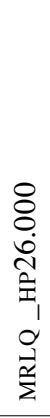 & 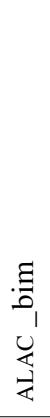 & 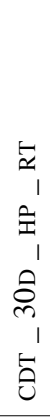 & 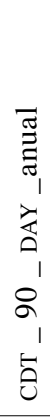 & $\begin{array}{l}\stackrel{\Xi}{\Xi_{1}} \\
\vec{z}_{\text {章 }}\end{array}$ & 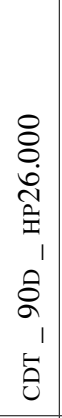 & 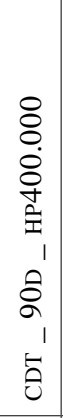 & 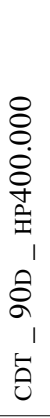 & 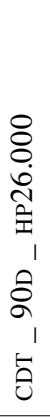 & 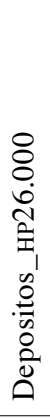 & 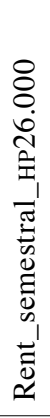 & 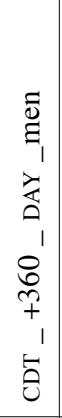 & 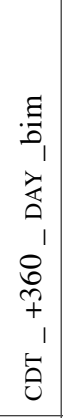 & 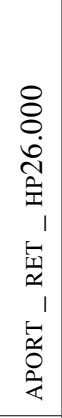 & 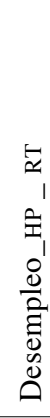 \\
\hline 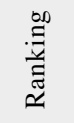 & હे & $\stackrel{\circ}{\sim}$ & $\bar{\nabla}$ & $\stackrel{\sim}{\sim}$ & $\stackrel{m}{\sim}$ & $\underset{\sim}{\Delta}$ & $\stackrel{n}{\sim}$ & $\stackrel{0}{\sim}$ & $\vec{\sim}$ & $\frac{\infty}{\sim}$ & $\stackrel{\partial}{\vec{\lambda}}$ & તิ & $\overrightarrow{\widetilde{N}}$ & తి & 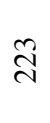 & $\stackrel{\mathbb{Z}}{\underset{N}{*}}$ & $\stackrel{\sim}{\tilde{N}}$ & స్ & $\widehat{ָ}$ \\
\hline
\end{tabular}




\begin{tabular}{|c|c|c|c|c|c|c|c|c|c|c|c|c|c|c|c|}
\hline 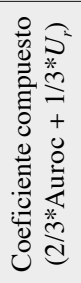 & $\begin{array}{l}\bar{\sigma} \\
\hat{n} \\
\bar{n} \\
0 \\
0 \\
0\end{array}$ & 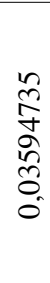 & 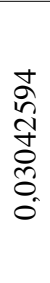 & \begin{tabular}{l}
$\infty$ \\
0 \\
0 \\
0 \\
$\stackrel{0}{0}$ \\
\multirow{2}{0}{} \\
0 \\
0
\end{tabular} & 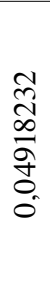 & \begin{tabular}{l}
$\frac{2}{\infty}$ \\
$\frac{\infty}{7}$ \\
\multirow{2}{*}{} \\
0 \\
0 \\
0
\end{tabular} & 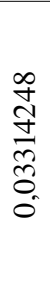 & 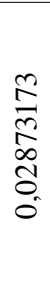 & 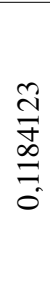 & $\begin{array}{l}\text { oे } \\
\text { Oे } \\
\text { ஸै } \\
0 \\
0 \\
0 \\
0\end{array}$ & $\begin{array}{l}\infty \\
0 \\
0 \\
0 \\
0 \\
+ \\
0 \\
0\end{array}$ & $\begin{array}{l}\infty \\
\infty \\
\infty \\
\stackrel{0}{0} \\
0 \\
0\end{array}$ & 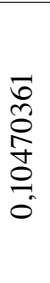 & $\begin{array}{l}\frac{\infty}{\omega} \\
\frac{\omega}{\infty} \\
\stackrel{0}{0} \\
0 \\
0\end{array}$ & 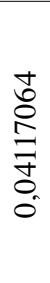 \\
\hline 茎 & $\begin{array}{l}n \\
2 \\
\dot{a} \\
\infty \\
\infty \\
\hat{n} \\
0\end{array}$ & 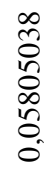 & $\begin{array}{l}\hat{b} \\
0 \\
\tilde{n} \\
0 \\
0 \\
0 \\
0\end{array}$ & $\begin{array}{l}\frac{g}{ \pm} \\
\frac{\vec{J}}{0} \\
\stackrel{m}{0}\end{array}$ & 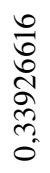 & 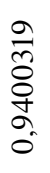 & $\begin{array}{l}n \\
\tilde{n} \\
\tilde{n} \\
\infty \\
\infty \\
\text { n. } \\
0\end{array}$ & 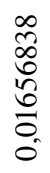 & 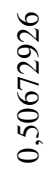 & 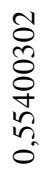 & 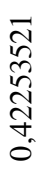 & ? & 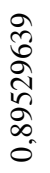 & 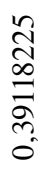 & 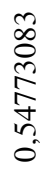 \\
\hline 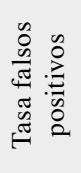 & 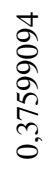 & 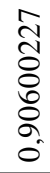 & 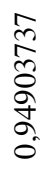 & 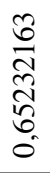 & $\begin{array}{l}n \\
\tilde{n} \\
n \\
\bar{\sigma} \\
0\end{array}$ & 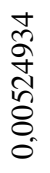 & $\begin{array}{l}0 \\
\text { cे } \\
0 \\
n \\
\infty \\
n \\
0\end{array}$ & 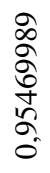 & 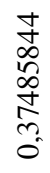 & 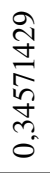 & 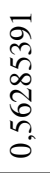 & $\begin{array}{l}\stackrel{1}{\infty} \\
\stackrel{\infty}{\sigma} \\
\infty \\
+ \\
+\end{array}$ & 0 & 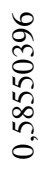 & 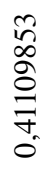 \\
\hline 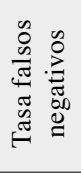 & 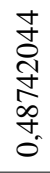 & 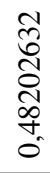 & 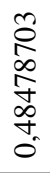 & $\begin{array}{l}0 \\
\swarrow 0 \\
0 \\
\infty \\
\infty \\
\infty \\
+ \\
0\end{array}$ & 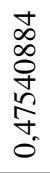 & 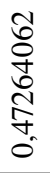 & \begin{tabular}{l}
0 \\
$\infty$ \\
$\infty$ \\
\multirow{1}{*}{} \\
$\infty$ \\
$\infty$ \\
+ \\
0
\end{tabular} & 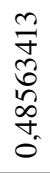 & 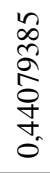 & 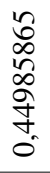 & 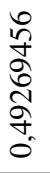 & 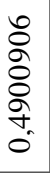 & 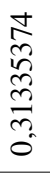 & 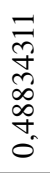 & 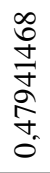 \\
\hline 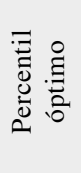 & 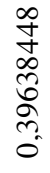 & 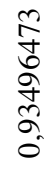 & 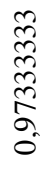 & 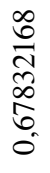 & 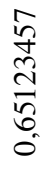 & $\begin{array}{l}\frac{n}{n} \\
\stackrel{n}{n} \\
a \\
0 \\
0\end{array}$ & $\begin{array}{l}\text { ত్రి } \\
\infty \\
\Xi \\
\bar{\Xi} \\
0\end{array}$ & 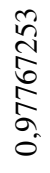 & \begin{tabular}{l}
$\hat{a}$ \\
$\hat{\circ}$ \\
$\hat{b}$ \\
o \\
\multirow{0}{0}{}
\end{tabular} & 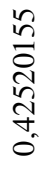 & 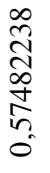 & 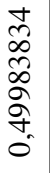 & 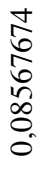 & $\begin{array}{l}\frac{n}{0} \\
\delta \\
\infty \\
\widetilde{\delta} \\
\dot{0} \\
0\end{array}$ & \begin{tabular}{l}
$\circ$ \\
8 \\
$\stackrel{2}{7}$ \\
$\stackrel{5}{7}$ \\
\multirow{2}{*}{}
\end{tabular} \\
\hline 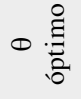 & $\tilde{n}$ & $\tilde{n}$ & $\ddot{0}$ & $\ddot{n}$ & $\ddot{0}$ & $\ddot{0}$ & $\tilde{o}$ & $\tilde{0}$ & $\tilde{0}$ & $\ddot{0}$ & $\ddot{n}$ & $\ddot{n}$ & $\begin{array}{l}6 \\
0 \\
0\end{array}$ & $\ddot{n}$ & $\tilde{0}$ \\
\hline $\begin{array}{l}\text { Oे } \\
\frac{3}{4}\end{array}$ & 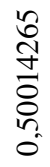 & 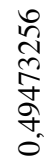 & \begin{tabular}{l}
\multirow{2}{0}{} \\
$\hat{0}$ \\
0 \\
0 \\
\multirow{2}{0}{} \\
0 \\
0
\end{tabular} & 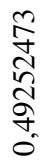 & 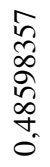 & $\begin{array}{l}\frac{a}{\hat{\sigma}} \\
\frac{\sigma}{\sigma} \\
\frac{\sigma}{+} \\
0\end{array}$ & 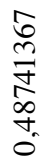 & 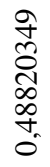 & 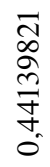 & 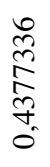 & 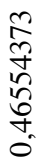 & 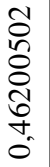 & $\begin{array}{l}\overrightarrow{\tilde{n}} \\
2 \\
\stackrel{\partial}{\vec{\sigma}} \\
\vec{\sigma} \\
\dot{0}\end{array}$ & 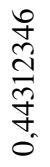 & 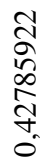 \\
\hline 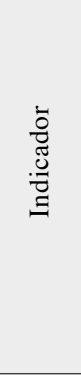 & $\begin{array}{l}8 \\
8 \\
0 \\
0 \\
\tilde{O} \\
\vdots \\
\sum^{\prime}\end{array}$ & 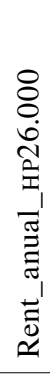 & 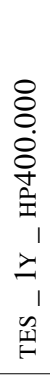 & $\begin{array}{l}\overline{0} \\
\Xi \\
\Xi \\
0 \\
\stackrel{0}{0} \\
0 \\
0 \\
0 \\
0\end{array}$ & $\begin{array}{l}\vec{\alpha} \\
1 \\
\vec{z} \\
\vec{y} \\
\underline{\underline{w}}\end{array}$ & 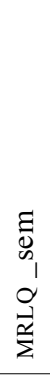 & 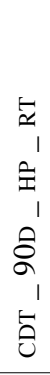 & 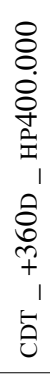 & 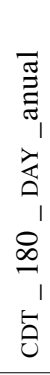 & 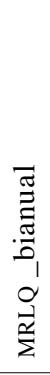 & 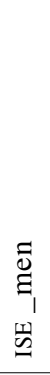 & $\begin{array}{l}. \Xi \\
\Xi \\
\end{array}$ & 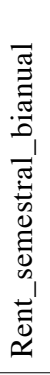 & 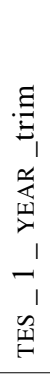 & 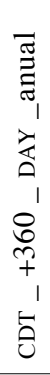 \\
\hline 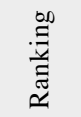 & $\stackrel{\infty}{\sim}$ & ते & હે & $\vec{\sim}$ & $\widetilde{\sim}$ & 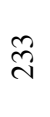 & ָे & $\tilde{\sim}$ & $\underset{\sim}{~}$ & $\hat{\vartheta}$ & $\stackrel{\infty}{\sim}$ & ले & $\stackrel{\stackrel{+}{\sim}}{\sim}$ & $\vec{J}$ & $\stackrel{\sim}{\sim}$ \\
\hline
\end{tabular}

